\title{
Assinaturas dinâmicas de um sistema coerente com aplicações
}

\author{
José Alberto Ramos Flor \\ DissertaÇÃo APRESENTADA \\ $\mathrm{AO}$ \\ Instituto de Matemática e Estatística \\ DA \\ Universidade DE SÃo PaUlo \\ PARA \\ OBTENÇÃO DO TÍTULO \\ $\mathrm{DE}$ \\ Mestre em CiênCias
}

Programa: Estatística

Orientador: Prof. Dr. Vanderlei da Costa Bueno

Durante o desenvolvimento deste trabalho o autor recebeu auxílio financeiro da CNPq

São Paulo, dezembro de 2011 


\title{
Assinaturas dinâmicas de um sistema coerente com aplicações
}

\author{
Este corresponde à redação \\ final da dissertação devidamente corrigida \\ e defendida por José Alberto Ramos Flor \\ e aprovada pela Comissão Julgadora.
}

Banca Examinadora:

- Prof. Dr. Vanderlei da Costa Bueno - IME-USP.

- Prof. Dr. Oswaldo Luiz do Valle Costa - Escola Politécnica-USP.

- Prof. Dr. Nelson Ithiro Tanaka - IME-USP. 


\section{Agradecimentos}

Gostaria de aproveitar esta oportunidade para agradecer às pessoas e instituções que, ajudaram no desenvolvimento deste trabalho.

Primeiramente gostaria de agradecer ao IME, como instituição e grupo humano, que contribuiram para minha formação acadêmica.

Gostaria de agradecer em especial a meu orientador , Prof. Dr Vanderlei da Costa Bueno, por todo seu apoio e compreensão, pela disposição que teve para ouvir minhas perguntar e idéias, e em especial pela confiança depositada em mim.

Quero agradecer ao meu pai Jose Melquiades, a minha mãe Maria, por todo o amor, carinho e apoio que me dão. À minha tia Sara, que é como uma segunda mãe, aos meus irmãos Juan Carlos e Miguel, pela força que me dão, às minhas primas, aos meus primos, aos meus avós e toda minha familia.

E finalmente à Deus por iluminar-me e guiar-me sempre até nos momentos de escuridão. 


\section{Resumo}

Nesta dissertação estudamos os sistemas coerentes. Um sistema de engenharia é completamente caracterizado por uma função de estrutura que relaciona o tempo de vida do sistema com os tempos de vida de seus componentes. Nos últimos sete anos, analisou-se uma nova estrutura para tal relação: as assinaturas de um sistema coerente, proposta por Samaniego, sob a hipótese de que os tempos de vida dos componentes são independentes e identicamente distribuidos

$$
P(T>t)=\sum_{i=1}^{n} P\left(T=T_{(i)}\right) P\left(T_{(i)}>t\right)
$$

onde os $T_{(i)}$ são as estatísticas de ordem associada aos tempos de vida dos componentes $T_{i}$. Embora restritiva, esta estrutura é substancialmente mais útil por exemplo na comparação do desempenho de sistemas. Um tratamento detalhado da teoria pode ser encontrado em Samaniego [20], Kochar [14], Navarro [15].

O objetivo da dissertação é analisar esta estrutura em um contexto mais amplo que considera a dinâmica no tempo e a dependência estocástica, utilizando a teoria dos martingais para processos pontuais:

-Estendemos a noção de assinaturas para o caso em que os tempos de vidas dos componentes são dependentes sobre diferentes níveis de informação, e recuperamos a definição clássica sob a hipótese de independência;

-Analisamos a importância da confiabilidade de um componente quando a falha do sistema coincida com a $i$-ésima falha ordenada dos componentes;

-Apresentamos uma nova aplicação das assinaturas no cálculo do tempo de vida residual do sistema coerente, baseada na teoria de valores extremos.

Palavras-chave: assinatura do sistema, assinatura dinâmica, sistema coerente, martingal, processo pontual, valores extremos. 


\section{Abstract}

A physical system is completely characterized by a structure function that relates the system lifetime with its components lifetimes. Over the past seven years, a new structure for such relationship was analyzed, the signature, proposed by Samaniego, which considers independent and identically distributed components lifetimes:

$$
P(T>t)=\sum_{i=1}^{n} P\left(T=T_{(i)}\right) P\left(T_{(i)}>t\right)
$$

where $T_{(i)}$ are the order statistics of the components lifetimes $T_{i}$ and $T$ is the system lifetime. Although restrictive, this structure is substantially more useful. A detailed treatment of the theory can be found in Samaniego [20], Kochar [14], Navarro [15].

The main goal in this work is to analyze the signature structure in a broader context, considering time dynamics and stochastic dependence, using the point processes martingale theory:

-We extend the notion of signatures for the case where the component lifetimes are dependent under different levels of information, and we recover the classical definition of signature under independence assumption;

-We analyze the reliability importance of a component when the failure of the system coincides with the $i$-th ordered failure of components;

-We present a new application of signatures. The calculation of the coherent system residual lifetime, based on extreme value theory.

Keywords: signatures of the system, dynamic signatures, coherent systems, martingales, point processes, extreme values. 


\section{Sumário}

1 Assinatura Clássica e Independência $\quad 1$

1.1 Assinatura de um Sistema Coerente . . . . . . . . . . . . . . . . 1

1.2 Assinatura Dinâmica . . . . . . . . . . . . . . . . . . . . 5

2 Assinatura Dinâmica e Dependência $\quad 9$

2.1 Representação dos Tempos de Vida através de Compensadores . . . . . . . . . . . 9

2.2 Assinatura Dinâmica ao nível de Informação $\mathfrak{F}_{t} \ldots \ldots \ldots$. . . . . . . . . . . . 14

2.3 Assinatura Dinâmica ao nível de Informação Completa $\mathfrak{F}^{*} \ldots$. . . . . . . . . . . . . . 19

3 Aplicações $\quad 29$

3.1 Importância de Barlow-Proschan e Assinatura . . . . . . . . . . . . . . . . . . 29

3.2 Importância de Componentes e Transformação no Compensador . . . . . . . . . . . 34

3.2.1 Importância de Natvig . . . . . . . . . . . . . . . . . . . . . . . . 37

3.3 Teoria de Valores Extremos e Assinaturas . . . . . . . . . . . . . . . . . . . . 44

4 Conclusões $\quad 53$

4.1 Considerações Finais . . . . . . . . . . . . . . . . . . . . . 53

4.2 Sugestões para Pesquisas Futuras . . . . . . . . . . . . . . . . 53

A Apêndice $\quad 55$

A.1 Processos Estocásticos . . . . . . . . . . . . . . . . . . . 55

A.1.1 Tempo de Parada . . . . . . . . . . . . . . . . . 56

A.1.2 Martingal . . . . . . . . . . . . . . . . . 57

A.2 Processos Pontuais . . . . . . . . . . . . . . . . . . . . 59

A.2.1 Fórmula Exponencial e Teorema de Girsanov . . . . . . . . . . . . . . . 62

A.3 Filtragens associadas aos Processos Pontuais . . . . . . . . . . . . . 70

$\begin{array}{ll}\text { Referências Bibliográficas } & 71\end{array}$ 


\section{Capítulo 1}

\section{Assinatura Clássica e Independência}

\subsection{Assinatura de um Sistema Coerente}

Como em Barlow and Proschan [4] um sistema de engenharia é completamente caracterizado pela sua função de estrutura $\Phi$ que relaciona o tempo de vida $T$ do sistema com os tempos de vida $T_{j}, j=1, . ., n$, de seus componentes definidos em um espaço de probabilidade completo $(\Omega, \mathfrak{F}, P)$,

$$
T=\Phi\left(T_{1}, T_{2}, . ., T_{n}\right)
$$

O sistema físico é mal modelado, se a melhora do desempenho de um de seus componentes causa a piora no seu funcionamento. Assim nossa atenção é restrita a funções de estruturas monótona crescente em cada uma de suas coordenadas. Além disso, para evitar trivialidades, eliminaremos aqueles sistemas cujo estado não depende do estado de funcionamento de cada um de seus componentes. Um sistema é chamado de coerente se a sua função de estrutura $\Phi$ é crescente e cada componente é relevante, isto é, se existe um tempo $t$ e uma configuração de $T$ no tempo $t$, tal que o sistema funciona se, e somente se, o componente funciona naquele instante.

O desempenho do sistema coerente pode ser medido pela relação entre a função de estrutura e as funções de distribuições dos tempos de vida dos componentes. A função de estrutura é uma maneira de indexar classes de sistemas coerentes mas tal representação torna-se inconveniente quando nosso objetivo é calcular a distribuição do tempo de vida do sistema. Uma representação alternativa para a função de distribuição do sistema coerente é através da assinatura do sistema, como em Samaniego [20], que, embora em contexto mais restrito do que a função de estrutura, é substancialmente mais útil. De Samaniego [21] temos o teorema:

Teorema 1.1.1 Seja $\left(T_{1}, . ., T_{n}\right)$ o vetor dos tempos de vida dos componentes de um sistema coerente $\Phi$, de ordem $n$, independentes e identicamente distribuidos com função de distribuição contínua $F$, e seja $T$ o tempo de vida do sistema coerente $\Phi$. Então :

$$
P(T>t)=\sum_{i=1}^{n} \alpha_{i} P\left(T_{(i)}>t\right)
$$

onde $\alpha_{i}=P\left(T=T_{(i)}\right)$ e $\left\{T_{(i)}, 1 \leq i \leq n\right\}$ são as estatísticas de ordem de $\left\{T_{i}, 1 \leq i \leq n\right\}$. 


\section{Demonstração}

Seja $\pi$ uma permutação dos inteiros positivos em $\{1, \ldots, n\}$ e $A_{i}$ o conjunto das permutações tal que a $i$-ésima componente coincide com o tempo de vida do sistema $T$, isto é:

$$
A_{i}=\left\{\pi \in \mathfrak{P}(n): T=T_{\pi(i)}, T_{\pi(1)}<T_{\pi(2)}<\ldots<T_{\pi(n)}\right\}
$$

onde $\mathfrak{P}(n)$ é o conjunto de todas as permutações de $\{1, \ldots, n\}$. Note que $T_{\pi(i)}=T_{(i)}$. Então

$$
\begin{aligned}
P(T>t) & =\sum_{i=1}^{n} P\left(T>t, \pi \in A_{i}\right) \\
& =\sum_{i=1}^{n} \sum_{\pi \in A_{i}} P\left(T>t, T=T_{\pi(i)}, T_{\pi(1)}<T_{\pi(2)}<\ldots<T_{\pi(n)}\right) \\
& =\sum_{i=1}^{n} \sum_{\pi \in A_{i}} P\left(T_{\pi(i)}>t, T=T_{(i)}, T_{\pi(1)}<T_{\pi(2)}<\ldots<T_{\pi(n)}\right) \\
& =\sum_{i=1}^{n} \sum_{\pi \in A_{i}} P\left(T_{(i)}>t, T=T_{(i)}, T_{\pi(1)}<T_{\pi(2)}<\ldots<T_{\pi(n)}\right) .
\end{aligned}
$$

Contudo os eventos $\left\{T=T_{(i)}>t\right\}$ e $\left\{T_{\pi(1)}<T_{\pi(2)}<\ldots<T_{\pi(n)}\right\}$ são independentes porque o primeiro evento depende somente da estatística de ordem $T_{(i)}$ e o segundo evento depende das ordenações das observações originais $T_{1}, \ldots, T_{n}$.

Assim temos

$$
\begin{aligned}
P(T>t) & =\sum_{i=1}^{n} P\left(T_{(i)}>t\right) \sum_{\pi \in A_{i}} P\left(T=T_{(i)}, T_{\pi(1)}<T_{\pi(2)}<\ldots<T_{\pi(n)}\right) \\
& =\sum_{i=1}^{n} P\left(\pi \in A_{i}\right) P\left(T_{(i)}>t\right) \\
& =\sum_{i=1}^{n} \alpha_{i} P\left(T_{(i)}>t\right)
\end{aligned}
$$

onde a terceira linha segue do fato que $P\left(\pi \in A_{i}\right)=\sum_{\pi \in A_{i}} P\left(T=T_{(i)}, T_{\pi(1)}<T_{\pi(2)}<\ldots<T_{\pi(n)}\right)$.

No contexto acima Samaniego [20] define:

Definição 1.1.1 Seja T o tempo de vida de um sistema coerente de ordem $n$ com os tempos de vida dos componentes $T_{1}, . ., T_{n}$, independentes e identicamente distribuidos com função de distribuição contínua $F$. $O$ vetor de assinatura $\alpha$ é definido como: 


$$
\alpha=\left(\alpha_{1}, \ldots, \alpha_{n}\right)
$$

onde $\alpha_{i}=P\left(T=T_{(i)}\right)$ e $\left\{T_{(i)}, 1 \leq i \leq n\right\}$ são as estatísticas de ordem de $\left\{T_{i}, 1 \leq i \leq n\right\}$.

Cada componente $\alpha_{i}$ do vetor de assinatura pode ser calculado como

$$
\alpha_{i}=\frac{\text { número de ordenações para as quais a i-ésima falha causa a falha do sistema }}{n !}
$$

onde $n$ ! é o numero total de ordenações de $n$ elementos.

\section{Exemplo 1.1.1}

a) Sistema em série.

Em um sistema em série, a primeira falha entre os componentes causa a falha do sistema, e assim, $\alpha_{1}=P\left(T=T_{(1)}\right)=1$ e os outros componentes do vetor de assinatura são zeros.

b) Sistema em paralelo.

Em um sistema em paralelo, o sistema falha se todos os componentes falham, e portanto, temos que $\alpha_{n}=P\left(T=T_{(n)}\right)=1$ enquanto os outros componentes do vetor de assinatura tomam o valor zero.

c) Sistema k-de-n.

Neste caso, o sistema falha se pelo menos $k$ componentes falham, assim $\alpha_{k}=P\left(T=T_{(k)}\right)=1$ e os resto dos componentes do vetor de assinatura são zeros.

As assinaturas são de grande utilidade na análise de confiabilidade pois no contexto de componentes com tempos de vida independentes e identicamente distribuidos com função de distribuição contínua, elas são independentes da distribuição dos tempos de vida dos componentes, e dependem unicamente das características da modelagem estrutural do sistema.

Um tratamento detalhado da teoria e aplicações das assinaturas pode ser encontrado em Samaniego [20]. Esta referência justifica detalhadamente por que da necessidade da hipótese de que os tempos de vida dos componentes são independentes e identicamente distribuidos. A propósito existem aplicações em que tal suposição é apropriada, como em baterias de iluminação, plaquetas ou chips de um computador digital, o subsistema de velas de ignição em um motor de automóvel. Em tais casos, o uso de assinaturas do sistema para as comparações entre os sistemas é apropriado e correto.

A utilidade das assinaturas em comparar o desempenho dos sistemas com componentes independentes e identicamente distribuidos deriva em grande parte dos resultados de representação e de preservação. Antes de comentar tais resultados, recordamos uma forma padrão de comparação estocástica. 
Definição 1.1.2 Sejam $T_{1}$ e $T_{2}$ duas variáveis aleátorias positivas.

A variável $T_{1}$ é estocasticamente menor que $T_{2}$, denotado por $T_{1} \leq_{s t} T_{2}$, se para todo $t \geq 0$

$$
P\left(T_{1}>t\right) \leq P\left(T_{2}>t\right)
$$

O resultado seguinte mostra que essa relação entre as assinaturas assegura uma relação semelhante entre os tempos de vida dos sistemas correspondentes.

Teorema 1.1.2 Sejam $\alpha_{1}$ e $\alpha_{2}$ as assinaturas de dois sistemas coerentes, ambos com $n$ componentes e tal que os tempos de vida dos componentes dos dois sistemas sejam independentes e identicamente distribuidos com a mesma distribuição $F$. Se $S_{1}$ e $S_{2}$ representam os tempos de vida dos sistemas coerentes com assinaturas $\alpha_{1}$ e $\alpha_{2}$, respectivamente, então:

$$
\alpha_{1} \leq_{s t} \alpha_{2} \rightarrow S_{1} \leq_{s t} S_{2}
$$

\section{Demonstração}

Escreveremos de novo a expressão $P(S>t)=\sum_{i=1}^{n} \alpha_{i} P\left(S_{(i)}>t\right)$, onde $S$ é um tempo de vida do sistema e $S_{(i)}$ são as estatísticas de ordem associadas aos componentes. Usando o fato que os tempos de vida dos componentes são independentes e identicamente distribuidos com distribuição $F$, a probabilidade $P\left(S_{(i)}>t\right)$ pode ser escrita como

$$
P\left(S_{(i)}>t\right)=\sum_{k=0}^{i-1}\left(\begin{array}{l}
n \\
k
\end{array}\right)(F(t))^{k}(1-F(t))^{n-k} .
$$

Portanto

$$
\begin{aligned}
P(S>t) & =\sum_{i=1}^{n} \alpha_{i} P\left(S_{(i)}>t\right) \\
& =\sum_{i=1}^{n} \alpha_{i} \sum_{k=0}^{i-1}\left(\begin{array}{l}
n \\
k
\end{array}\right)(F(t))^{k}(1-F(t))^{n-k} \\
& =\sum_{k=0}^{n-1}\left(\sum_{i=k+1}^{n} \alpha_{i}\right)\left(\begin{array}{l}
n \\
k
\end{array}\right)(F(t))^{k}(1-F(t))^{n-k} .
\end{aligned}
$$

Usando a hipótese $\alpha_{1} \leq s t \alpha_{2}$ temos que $\sum_{i=k+1}^{n} \alpha_{1 i} \leq \sum_{i=k+1}^{n} \alpha_{2 i}$ para todo $k \geq 0$ e assim, para cada $t \geq 0$

$$
P\left(S_{1}>t\right)=\sum_{k=0}^{n-1}\left(\sum_{i=k+1}^{n} \alpha_{1 i}\right)\left(\begin{array}{l}
n \\
k
\end{array}\right)(F(t))^{k}(1-F(t))^{n-k}
$$




$$
\begin{aligned}
& \leq \sum_{k=0}^{n-1}\left(\sum_{i=k+1}^{n} \alpha_{2 i}\right)\left(\begin{array}{l}
n \\
k
\end{array}\right)(F(t))^{k}(1-F(t))^{n-k} \\
& =P\left(S_{2}>t\right)
\end{aligned}
$$

o que é equivalente a $S_{1} \leq_{s t} S_{2}$

Outra questão importante é o fato de que podemos estar interessados em comparar sistemas de tamanhos diferentes. Embora tal comparação possa surgir em geral, a comparação é relevante quando envolve sistemas com componentes novos e usados. O teorema anterior não se aplica a tal problema.

A relação exata entre a assinatura de um sistema de ordem $n$ com a assinatura de um sistema de ordem maior, com mesma funçaõ de distribuição, de tempo de vida, foi caracterizada pelo seguinte teorema devido a Samaniego:

Teorema 1.1.3 Seja $\alpha=\left(\alpha_{1}, . ., \alpha_{n}\right)$ o vetor de assinatura de um sistema coerente cujos tempos de vida dos componentes são independentes e identicamente distribuidos com distribuição contínua $F$. Então o tempo de vida de um sistema de $(n+1)$-componentes cujos componentes têm tempos de vida independentes e identicamente distribuidos com distribuição $F$ e com assinatura $\alpha^{*}$, onde

$$
\alpha_{k}^{*}=\frac{(n-k+1) \alpha_{k}+(k-1) \alpha_{k-1}}{n+1}
$$

para todo $k, 1 \leq k \leq n+1$ tem a mesma função de distribuição do tempo de vida do sistema com $n$-componentes e assinatura $\alpha$.

Demonstração.Ver Samaniego [22].

Koshar [14] e Shaked [23] estenderam o conceito de assinatura para o caso em que os tempos de vida dos componentes de um sistema são permutáveis ( i.e a ditribuição conjunta $F\left(t_{1}, t_{2}, . ., t_{n}\right.$ ), de $\left(T_{1}, T_{2}, . ., T_{n}\right)$, é a mesma para qualquer permutação dos $\left.t_{1}, t_{2}, \ldots, t_{n}\right)$, um caso de interesse e de applicações práticas na teoria de confiabilidade.

\subsection{Assinatura Dinâmica}

Navarro [15] e Samaniego [19] consideram assinaturas dinâmicas (condicionadas) na comparação da confiabilidade de sistemas novos e usados. Em suas abordagens consideram o tempo de vida do sistema condicionado a eventos dependentes do tempo. Por exemplo Navarro [15] considerou os eventos $\{T>t\}$ e $\{T>t\} \cap\left\{T_{(i)} \leq t\right\}$ com assinaturas $P\left(T=T_{(i)} \mid\{T>t\}\right)$ e $P\left(T=T_{(i)} \mid\{T>\right.$ $\left.t\} \cap\left\{T_{(i)} \leq t\right\}\right)$, respectivamente. Infelizmente , em ambas situações, a assinatura do sistema depende da distribuição $F$ e do tempo $t$.

Samaniego [19] considerou o evento $\{T>t\} \cap\left\{T_{(i)} \leq t<T_{(i+1)}\right\}$ e propôs a assinatura do sistema como $P\left(T=T_{(k)} \mid\{T>t\} \cap\left\{T_{(i)} \leq t<T_{(i+1)}\right\}\right)$ que não depende da distribuição $F$ ou do tempo $t$ e 
possui as propriedades usuais das assinaturas. Nesta seção detalharemos as principais propriedades da assinatura dinâmica proposta por Samaniego [19].

Definição 1.2.1 A assinatura dinâmica do sistema no tempo t é o vetor de dimensão $n-i$, cujos componentes são

$$
\beta_{k}=P\left(T=T_{(k)} \mid\{T>t\} \cap\left\{T_{(i)} \leq t<T_{(i+1)}\right\}\right)
$$

com $k=i+1, i+2, . ., n$.

Quando o evento $\{T>t\} \cap\left\{T_{(i)} \leq t<T_{(i+1)}\right\}$ tem probabilidade zero a assinatura dinâmica não está definida. O principal teorema prova que se o evento $\{T>t\} \cap\left\{T_{(i)} \leq t<T_{(i+1)}\right\}$ tem probabilidade positiva, cada componente da assinatura dinâmica é independente da distribuição $F$ e do tempo $t$, e dado pela fórmula

$$
\beta_{k}=\frac{\alpha_{k}}{\sum_{j=i+1}^{n} \alpha_{j}}
$$

onde $\alpha=\left(\alpha_{1}, . ., \alpha_{n}\right)$ é a assinatura do sistema.

Para demonstrar tal teorema usaremos o seguinte lema:

Lema 1.2.1 Seja $\alpha$ a assinatura de um sistema coerente de ordem n. Seja $T$ o tempo de vida do sistema e $T_{(i)}$ a $i$-ésima estatística de ordem dos tempos de vida dos componentes, independentes e identicamente distribuidos com função de distribuição contínua $F$. Então para $i<n$

$$
P\left(T>t \mid\left\{T_{(i)} \leq t<T_{(i+1)}\right\}\right)=\sum_{j=i+1}^{n} \alpha_{j}
$$

\section{Demostração}

Por hipótese os tempos de falha dos componentes não ocorrem simultâneamente:

$$
\begin{aligned}
P\left(T>t \mid\left\{T_{(i)} \leq t<T_{(i+1)}\right\}\right) & =\sum_{j=1}^{n} P\left(T>t, T=T_{(j)} \mid\left\{T_{(i)} \leq t<T_{(i+1)}\right\}\right) \\
& =\sum_{j=i+1}^{n} P\left(T>t, T=T_{(j)} \mid\left\{T_{(i)} \leq t<T_{(i+1)}\right\}\right) .
\end{aligned}
$$

Analisaremos cada parcela da última expressão.

$$
\begin{gathered}
P\left(T>t, T=T_{(j)} \mid\left\{T_{(i)} \leq t<T_{(i+1)}\right\}\right)= \\
=P\left(T>t \mid\left\{T=T_{(j)}\right\} \cap\left\{T_{(i)} \leq t<T_{(i+1)}\right\}\right) P\left(T=T_{(j)} \mid\left\{T_{(i)} \leq t<T_{(i+1)}\right\}\right)
\end{gathered}
$$




$$
\begin{aligned}
& =P\left(T_{(j)}>t \mid\left\{T=T_{(j)}\right\} \cap\left\{T_{(i)} \leq t<T_{(i+1)}\right\}\right) P\left(T=T_{(j)} \mid\left\{T_{(i)} \leq t<T_{(i+1)}\right\}\right) \\
& =P\left(T_{(j)}>t \mid\left\{T=T_{(j)}\right\} \cap\left\{T_{(i)} \leq t<T_{(i+1)}\right\}\right) P\left(T=T_{(j)} \mid\left\{T_{(i)} \leq t<T_{(i+1)}\right\}\right) \\
& =P\left(T_{(j)}>t \mid\left\{T=T_{(j)}\right\} \cap\left\{T_{(i)} \leq t<T_{(i+1)}\right\}\right) P\left(T=T_{(j)}\right) \\
& =P\left(T_{(j)}>t \mid\left\{T_{(i)} \leq t<T_{(i+1)}\right\}\right) \alpha_{j}
\end{aligned}
$$

nesse estágio usamos o fato que os $\left\{T_{k}, k=1, \ldots, n\right\}$ são independentes e identicamente distribuidos, o que implica que os eventos $\left\{T_{(i)} \leq t<T_{(i+1)}\right\}$ e $\left\{T=T_{(j)}\right\}$ são independentes ${ }^{1}$. Para finalizar notemos que $P\left(T_{(j)}>t \mid\left\{T_{(i)} \leq t<T_{(i+1)}\right\}\right)=1$ para $j \in\{i+1, i+2, . ., n\}$. Assim concluimos que

$$
P\left(T>t \mid\left\{T_{(i)} \leq t<T_{(i+1)}\right\}\right)=\sum_{j=i+1}^{n} \alpha_{j}
$$

terminando a demonstração.

Teorema 1.2.1 Seja $\alpha$ a assinatura de um sistema coerente de ordem $n$. Seja T o tempo de vida do sistema e $T_{(i)}$ a $i$-ésima estátística de ordem dos tempos de vidas dos componentes, independentes e identicamente distribuidos com função de distribuição contínua F. Suponhamos que cada $\left\{T_{(i)} \leq\right.$ $\left.t<T_{(i+1)}\right\}$ tem probabilidade positiva. Então a assinatura dinâmica do sistema, $\beta$, dado o evento $\left\{T_{(i)} \leq t<T_{(i+1)}\right\}$, é um vetor $(n-i)$-dimensional com componentes dado pela formula

$$
\beta_{k}=P\left(T=T_{(k)} \mid\{T>t\} \cap\left\{T_{(i)} \leq t<T_{(i+1)}\right\}\right)=\frac{\alpha_{k}}{\sum_{j=i+1}^{n} \alpha_{j}}
$$

para $k=i+1, i+2, . ., n$.

\section{Demonstração}

Calcularemos a probabilidade condicional $P\left(T=T_{(k)} \mid\{T>t\} \cap\left\{T_{(i)} \leq t<T_{(i+1)}\right\}\right)$ :

$$
\begin{aligned}
P\left(T=T_{(k)} \mid\{T>t\} \cap\left\{T_{(i)} \leq t<T_{(i+1)}\right\}\right) & =\frac{P\left(T=T_{(k)} \cap\{T>t\} \cap\left\{T_{(i)} \leq t<T_{(i+1)}\right\}\right.}{P\left(\{T>t\} \cap\left\{T_{(i)} \leq t<T_{(i+1)}\right\}\right)} \\
& =\frac{P\left(T=T_{(k)} \cap\{T>t\} \mid\left\{T_{(i)} \leq t<T_{(i+1)}\right\}\right)}{P\left(T>t \mid\left\{T_{(i)} \leq t<T_{(i+1)}\right\}\right)} \\
& =\frac{\alpha_{k} P\left(T_{(k)}>t \mid\left\{T_{(i)} \leq t<T_{(i+1)}\right\}\right)}{P\left(T>t \mid\left\{T_{(i)} \leq t<T_{(i+1)}\right\}\right)} \\
& =\frac{\alpha_{k}}{\sum_{j=i+1}^{n} \alpha_{j}}
\end{aligned}
$$

onde a terceira igualdade se justifica pela independência de $\left\{T_{(i)} \leq t<T_{(i+1)}\right\}$ e $\left\{T=T_{(k)}\right\}$, e a última igualdade decorre do lema anterior e do fato que $P\left(T_{(k)}>t \mid\left\{T_{(i)} \leq t<T_{(i+1)}\right\}\right)=1$ para

\footnotetext{
${ }^{1} \mathrm{O}$ motivo da independência é que o primeiro evento depende somente da distribuição da estátística de ordem e não da modelagem do sistema enquanto o segundo depende da modelagem do sistema e não da função de distribuição $F$ dos componentes
} 
$k=i+1, i+2, . ., n$.

No que segue, $\beta_{n}(n-i)$ representa a assinatura com $n$ coordenadas equivalente à assinatura do sistema com $n$ componentes e $i$ falhas. Ver teorema 1.1.3 e Samaniego [19].

Teorema 1.2.2 Considere dois sistemas coerentes baseados em $n$ componentes com tempos de vida independentes e identicamente distribuidos com função de distribuição continua F. Suponha que ambos sistemas estejam funcionando e que exatamente $i$ e $j$ componentes falharam, respectivamente, em cada sistema no tempo $t$. Sejam $\beta_{n}(n-i)$ e $\beta_{n}(n-j)$ suas assinaturas dinâmicas de ordem $n$ e $S_{1}$ e $S_{2}$ os tempos de vida correspondente. Se $\beta_{n}(n-i) \leq_{s t} \beta_{n}(n-j)$ então

$$
\left(S_{1}-t \mid S_{1}>t, T_{(i)} \leq t<T_{(i+1)}\right) \leq_{s t}\left(S_{2}-t \mid S_{2}>t, T_{(i)} \leq t<T_{(i+1)}\right)
$$

Demonstração Ver Samaniego [19].

Nesta dissertação, no capítulo 1, introduzimos o conceito de assinaturas e descrevemos um resumo dos resultados atuais da teoria desenvolvida por diversos autores. No capítulo 2 abordamos os aspectos dinâmicos e estocásticos das assinaturas, generalizando os conceitos clássicos. Finalmente, no capítulo 3, analisamos algumas aplicações da teoria da confiabilidade no contexto das assinaturas. Estudamos a importância da confiabilidade dos componentes, os reparos mínimos através de compensadores e o calculo do tempo residual do sistema utilizando a teoria de valores extremos. No apêndice delineamos os resultados da teoria de martingais para processos pontuais que utilizamos na dissertação. 


\section{Capítulo 2}

\section{Assinatura Dinâmica e Dependência}

Pretendemos dar uma nova abordagem para assinaturas de sistemas coerentes. Consideramos a evolução do sistema no tempo sob diferentes níveis de informação e para cada nível de informação apresentamos a assinatura dinâmica. Desta forma, se os tempos de vida dos componentes são independentes e identicamente distribuidos com distribuição contínua, a assinatura do sistema dinâmico tem a propriedade especial de que é independente de ambos, a distribuição $F$ e o tempo de inspeção $t$. Este fato vai além da mera simplicidade e aplicabilidade do vetor de assinaturas. Reflete as características da modelagem do sistema correspondente e pode ser utilizado na comparação do desempenho de sistemas.

Na seção 2.1 faremos uma breve apresentação dos principais tópicos de estudo na ánalise da evolução de um sistema coerente no tempo. Na seção 2.2, analisamos detalhadamente o tempo de vida do sistema dado o nível de informação $\mathfrak{F}_{t}$, gerado pelas ordenações dos tempos de vida dos componentes. Definimos uma assinatura dinâmica neste nível de informação e recuperamos a noção da assinatura clássica sob a hipótese em que os tempos de vida dos componentes são independentes e identicamente distribuidos. Na seção 2.3, um nível maior de informação é considerado, chamado informação completa, $\mathfrak{F}_{t}^{*}$, que em adição à informação $\mathfrak{F}_{t}$, contém a informação sobre o tempo de vida do sistema. Neste nível de informação um novo tipo de assinatura dinâmica é definido e, por sua vez, sob a hipótese de que os tempos de vida dos componentes são independentes e identicamente distribuidos recuperamos a noção de assinatura dinâmica definida por Samaniego [19].

Por simplicidade de notação vamos assumir que as relações entre conjuntos mensuráveis e variables aleatórias, como $\subset, \subseteq, \leq,<,=$ serão sempre com probabilidade um. Assim expressões ou termos como P-q.c geralmente serão omitidos. Para terminar, com respeito as esperanças condicionais não faremos distinção entre uma versão e sua classe de equivalência P-q.c .

\subsection{Representação dos Tempos de Vida através de Compensadores}

Consideremos um vetor de $n$ variáveis aleatórias $\left(T_{1}, . ., T_{n}\right)$ finitas, positivas, definidas em um espaço de probabilidade $(\Omega, \mathcal{F}, P)$ com a propriedade $P\left(T_{i} \neq T_{j}\right)=1,1 \leq i \neq j \leq n$. Cada variável $T_{i}$ representa o tempo de vida do componente $i$ de um sistema coerente $\Phi$ com componentes em $\mathcal{C}=\{1, \ldots, n\}$. A condição $P\left(T_{i} \neq T_{j}\right)=1,1 \leq i \neq j \leq n$ implica que não há falhas simultâneas nos componentes do sistema coerente. 
A evolução dos componentes no tempo define um processo pontual através dos tempos das falhas ordenados dos componentes e as marcas correspondentes.

Denotemos por $T_{(1)}<T_{(2)}<\ldots<T_{(n)}$ a ordenação dos valores das realizações dos $n$ tempos de vida dos componentes. As variáveis $X_{i}=\left\{k: T_{(i)}=T_{k}\right\}$ representam as marcas correspondentes a tal ordenação.

Por convenção adotamos que:

a) $T_{(n+1)}=T_{(n+2)}=\ldots . .=\infty$;

b) $X_{n+1}=X_{n+2}=\ldots=p$ onde p é uma marca fícticia diferente de $1, \ldots, \mathrm{n}$.

A sequência $\left(T_{(n)}, X_{n}, n \geq 1\right)$ define um processo pontual marcado.

O tempo de vida $T$ do sistema é monitorado ao nível de seus componentes, isto é, a cada instante de tempo $t$ observamos se o evento $\left\{T_{(i)} \leq t\right\}$ ocorre ou não, e se esse evento ocorre observamos qual componente falhou e o instante exato de falha. A formulação matématica de nossas observações é dada por uma familia de sub- $\sigma$-álgebra $\left(\mathfrak{F}_{t}, t \geq 0\right)$

$$
\mathfrak{F}_{t}=\sigma\left\{1\left(T_{(i)}>s\right), X_{i}=j, 1 \leq i \leq n, j \in \mathcal{C}, 0<s \leq t\right\}
$$

que satisfaz as condições de Dellacherie de continuidade à direita e completitude.

Em adição, consideramos o processo pontual $N(i) j$ onde

$$
N_{(i) j}(t)=1\left\{T_{(i)} \leq t, X_{i}=j\right\}
$$

o qual é um $\mathfrak{F}_{t}$-submartingal. Assim pela decomposição de Doob-Meyer para $N(i) j$ ( Ver teorema A.1.7), existe um único processo $\mathfrak{F}_{t}$-previsível, $\left(A_{(i) j}(t), t \geq 0\right)$, chamado $\mathfrak{F}_{t}$-compensador de $N_{(i) j}(t)$, com $A_{(i) j}(0)=0$, e tal que $N_{(i) j}(t)-A_{(i) j}(t)$ é um $\mathfrak{F}_{t}$-martingal. Sob a hipótese que $T_{i}$ seja um tempo de parada totalmente inacessível o $\mathfrak{F}_{t}$-compensador $A_{(i) j}(t)$ é contínuo.

A idéia intuitiva do compensador é a de que ele generaliza a noção clássica de risco. Representa a propensão de falha no instante $t$, condicionada à informação passada, excluindo o presente. Ver Arjas [1].

A contagem das falhas do componente $j, N_{j}(t)$, é dada por $N_{j}(t)=\sum_{i \geq 1} N_{(i) j}(t)$ e seu $\mathfrak{F}_{t^{-}}$ compensador $A_{j}(t)$ é dado por $A_{j}(t)=\sum_{i \geq 1} A_{(i) j}(t)$ desde que a decomposição de Doob-Meyer é única e a soma finita de martingais é um martingal. Analogamente, a contagem da $i$-falha, $N_{(i)}(t)$, é dada por $N_{(i)}(t)=\sum_{j \in \mathcal{C}} N_{(i) j}(t)$ e, pelas mesmas razões, o $\mathfrak{F}_{t}$-compensador $A_{(i)}(t)$ é dado por $A_{(i)}(t)=\sum_{j \in \mathcal{C}} A_{(i) j}(t)$. 
Dado o sistema coerente $(\Phi, \mathcal{C})$ com tempo de vida $T$, queremos calcular o $\mathfrak{F}_{t}$-compensador $\left(A_{\Phi}(t), t \geq 0\right)$, associado ao processo pontual $N_{\Phi}(t)=1(T \leq t)$, como função dos compensadores correspondentes ao tempos de vida dos componentes. Para tanto, a definição do nível crítico para cada falha ordenada é essencial.

Definição 2.1.1 O nível crítico do componente $j$ para que a falha do sistema coincida com a $i$ ésima falha dos componentes, $Y_{(i) j}$, é o primeiro instante, a partir do qual a falha do componente $j$, no instante $T_{(i)}$, causa a falha do sistema.

Com tal definição exibimos uma decomposição para o $\mathfrak{F}_{t}$-compensador $A_{\Phi}(t)$ do sistema, que usaremos nas seções posteriores.

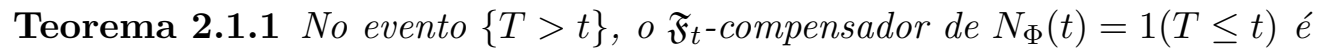

$$
A_{\Phi}(t)=\sum_{i=1}^{n} \sum_{j=1}^{n}\left[A_{(i) j}(t)-A_{(i) j}\left(Y_{(i) j}\right)\right]^{+}
$$

onde $a^{+}=\max \{a, 0\}$.

\section{Demonstração}

Como $A_{(i) j}(t)$ é o $\mathfrak{F}_{t}$-compensador de $N_{(i) j}(t)$ temos que

$$
E\left[\int_{0}^{\infty} C_{(i) j}(s) d N_{(i) j}(s)\right]=E\left[\int_{0}^{\infty} C_{(i) j}(s) d A_{(i) j}(s)\right]
$$

para todo processo não negativo $\mathfrak{F}_{t}$-previsível $\left(C_{(i) j}(t), t \geq 0\right)$. Seja $(C(t), t \geq 0)$ qualquer processo não negativo $\mathfrak{F}_{t}$-previsível. A partir dele, definimos um novo processo $\mathfrak{F}_{t}$-previsível $C_{(i) j}(s)=$ $C(s) 1\left(Y_{(i) j}<s \leq t \wedge T\right)$ com $t$ fixo. Pela definição de compensador temos :

$$
\begin{aligned}
& E\left[\int_{0}^{\infty} C(s) 1\left(Y_{(i) j}<s \leq t \wedge T\right) d N_{(i) j}(s)\right] \\
= & E\left[\int_{0}^{\infty} C(s) 1\left(Y_{(i) j}<s \leq t \wedge T\right) d A_{(i) j}(s)\right] .
\end{aligned}
$$

Notemos que, por hipótese, o tempo de vida do sistema $T$ deve coincidir com algum $T_{(i)}$, para $\operatorname{algum} i$, e sua falha deve coincidir com a falha de algum componente $j \in \mathcal{C}$. Assim, em um intervalo infinitesimal $d s$,

$$
\{T \in d s\}=\bigcup_{T_{(i)}>Y_{(i) j}}\left\{T_{(i)} \in d s, X_{i}=j\right\} .
$$

Escrevemos

$$
d N_{\Phi}(s)=\sum_{i=1}^{n} \sum_{j=1}^{n} 1\left(Y_{(i) j}<s \leq t \wedge T\right) d N_{(i) j}(s)
$$

pois ao integrar $d N_{\Phi}(s)$ temos 


$$
\begin{aligned}
N_{\Phi}(t) & =\int_{0}^{\infty} \sum_{i=1}^{n} \sum_{j=1}^{n} 1\left(Y_{(i) j}<s \leq t \wedge T\right) d N_{(i) j}(s) \\
& =\sum_{i=1}^{n} \sum_{j=1}^{n} 1\left(Y_{(i) j}<T_{i} \leq t \wedge T\right) 1\left(X_{i}=j\right) \\
& =\sum_{i=1}^{n} \sum_{j=1}^{n} 1\left(T_{(i)} \leq t, T=T_{(i)}, X_{i}=j\right) \\
& =1(T \leq t) .
\end{aligned}
$$

Dessa forma, para todo processo não negativo $C(s), \mathfrak{F}_{t}$-previsível

$$
\begin{aligned}
E\left[\int_{0}^{\infty} C(s) d N_{\Phi}(s)\right] & =E\left[\int_{0}^{\infty} C(s) \sum_{i=1}^{n} \sum_{j=1}^{n} 1\left(Y_{(i) j}<s \leq t \wedge T\right) d N_{(i) j}(s)\right] \\
& =\sum_{i=1}^{n} \sum_{j=1}^{n} E\left[\int_{0}^{\infty} C(s) 1\left(Y_{(i) j}<s \leq t \wedge T\right) d N_{(i) j}(s)\right] \\
& =\sum_{i=1}^{n} \sum_{j=1}^{n} E\left[\int_{0}^{\infty} C(s) 1\left(Y_{(i) j}<s \leq t \wedge T\right) d A_{(i) j}(s)\right] \\
& =E\left[\int_{0}^{\infty} C(s) \sum_{i=1}^{n} \sum_{j=1}^{n} 1\left(Y_{(i) j}<s \leq t \wedge T\right) d A_{(i) j}(s)\right]
\end{aligned}
$$

Portanto, da definição de $\mathfrak{F}_{t}$-compensador do sistema $\Phi$, temos que, no evento $\{T>t\}$ :

$$
\begin{aligned}
A_{\Phi}(t) & =\sum_{i=1}^{n} \sum_{j=1}^{n} \int_{0}^{\infty} 1\left(Y_{(i) j}<s \leq t \wedge T\right) d A_{(i) j}(s) \\
& =\sum_{i=1}^{n} \sum_{j=1}^{n} \int_{0}^{\infty} 1\left(Y_{(i) j}<s \leq t\right) d A_{(i) j}(s) \\
& =\sum_{i=1}^{n} \sum_{j=1}^{n}\left[A_{(i) j}(t)-A_{(i) j}\left(Y_{(i) j}\right)\right]^{+}
\end{aligned}
$$

concluindo a demonstração. 
O lema seguinte, que relaciona as esperanças condicionais envolvendo informações do tipo $\mathfrak{F}_{t} \cap$ $\left\{T_{(i)} \leq t<T_{(i+1)}\right\}$ e $\mathfrak{F}_{T_{(i)}} \cap\left\{T_{(i)} \leq t<T_{(i+1)}\right\}$, é importante para desenvolvimentos posteriores.

Lema 2.1.1 Sejam $T_{1}, . ., T_{n} \mathfrak{F}_{t}$-tempos de parada representando os tempos de vida dos componentes de um sistema coerente $\Phi$ com tempo de vida $T$. Denotemos $T_{(0)}=0$ e $T_{(n+1)}=\infty$. Então, ao nível de informação $\mathfrak{F}_{t}$, no evento $\{T>t\}$, temos que:

$$
P\left(T=T_{(k) j}, T_{(i)} \leq t<T_{(i+1)} \mid \mathfrak{F}_{t}\right)=P\left(T=T_{(k) j}, T_{(i)} \leq t<T_{(i+1)} \mid \mathfrak{F}_{T_{(i)}}\right)
$$

para todo $k \geq i+1$.

\section{Demonstração}

Se denotamos por $I_{i}$ o evento $\left\{T_{(i)} \leq t<T_{(i+1)}\right\}$ temos que, pelo lema A.2.1, quase-certamente com respeito a $P^{I_{i}}$

$$
\begin{gathered}
P\left(T=T_{(k) j}, T_{(i)} \leq t<T_{(i+1)} \mid \mathfrak{F}_{t}\right)=P^{I_{i}}\left(T=T_{(k) j} \mid I_{i} \cap \mathfrak{F}_{t}\right) \\
P\left(T=T_{(k) j}, T_{(i)} \leq t<T_{(i+1)} \mid \mathfrak{F}_{T_{(i)}}\right)=P^{I_{i}}\left(T=T_{(k) j} \mid I_{i} \cap \mathfrak{F}_{T_{(i)}}\right) .
\end{gathered}
$$

Como nesse intervalo $I_{i}=\left\{T_{(i)} \leq t<T_{(i+1)}\right\}$ a informação de $\mathfrak{F}_{T_{(i)}}$ é a mesma que $\mathfrak{F}_{t}$, isto é, $\mathfrak{F}_{t} \cap\left\{T_{(i)} \leq t<T_{(i+1)}\right\}=\mathfrak{F}_{T_{(i)}} \cap\left\{T_{(i)} \leq t<T_{(i+1)}\right\}$.

$$
P\left(T=T_{(k) j}, T_{(i)} \leq t<T_{(i+1)} \mid \mathfrak{F}_{t}\right)=P\left(T=T_{(k) j}, T_{(i)} \leq t<T_{(i+1)} \mid \mathfrak{F}_{T_{(i)}}\right)
$$

quase-certamente com respeito a $P^{I_{i}}$. Como ambas variáveis têm suporte em $I_{i}$, concluimos que

$$
P\left(T=T_{(k) j}, T_{(i)} \leq t<T_{(i+1)} \mid \mathfrak{F}_{t}\right)=P\left(T=T_{(k) j}, T_{(i)} \leq t<T_{(i+1)} \mid \mathfrak{F}_{T_{(i)}}\right)
$$

quase-certamente com respeito à probabilidade $P$.

\section{Observação 1}

Analogamente, podemos demostrar que para todo $k \geq i+1$

$$
P\left(T \geq T_{(k)}, T_{(i)} \leq t<T_{(i+1)} \mid \mathfrak{F}_{t}\right)=P\left(T \geq T_{(k)}, T_{(i)} \leq t<T_{(i+1)} \mid \mathfrak{F}_{T_{(i)}}\right)
$$




\subsection{Assinatura Dinâmica ao nível de Informação $\mathfrak{F}_{t}$}

Nesta seção a informação disponível é dada pelas $\sigma$-álgebras $\left\{\mathfrak{F}_{t}, t \geq 0\right\}$, que representam o sistema coerente monitorado ao nível de seus componentes. Intuitivamente, a cada instante de tempo $t$ observamos se a $i$-ésima falha ocorreu, ou não. Se ocorreu, conhecemos exatamente o instante de ocorrência e o componente que originou tal falha.

Se o sistema tem exatamente $i$ falhas, a evolução de $P\left(T>t \mid \mathfrak{F}_{t}\right)$ está relacionado com a propensão de falhar num tempo posterior $T_{(k)}$ devido a algum componente $j \in \mathcal{C}, P\left(T=T_{(k) j} \mid \mathfrak{F}_{t}\right)$. O próximo lema determina tal relação:

Lema 2.2.1 ( Decomposição de $P\left(T>t \mid \mathfrak{F}_{t}\right)$ ao nível de informação $\mathfrak{F}_{t}$ )

Seja $T$ o tempo de vida de um sistema coerente $\Phi$ de ordem $n$ e sejam $T_{1}, . ., T_{n}$ os $\mathfrak{F}_{t}$-tempos de parada representando os tempos de vida dos seus componentes com $T_{(0)}=0$ e $T_{(n+1)}=\infty$. Então, ao nível de informação $\mathfrak{F}_{t}$, no evento $\{T>t\}$, temos que:

$$
P\left(T>t \mid \mathfrak{F}_{t}\right)=\sum_{i=0}^{n} h(i, t) 1\left(T_{(i)} \leq t<T_{(i+1)}\right)
$$

onde

$$
h(i, t)=\sum_{k=i+1}^{n} \sum_{j=1}^{n} P\left(T=T_{(k) j} \mid \mathfrak{F}_{t}\right) 1\left(T_{(k) j}>t\right)
$$

para $i=0,1, . ., n-1$ e $h(n)=0$.

\section{Demonstração}

Como os componentes não falham simultâneamente temos

$$
P\left(T>t \mid \mathfrak{F}_{t}\right)=E\left[1(T>t) \mid \mathfrak{F}_{t}\right]=\sum_{i=0}^{n} E\left[1(T>t) 1\left(T_{(i)} \leq t<T_{(i+1)}\right) \mid \mathfrak{F}_{t}\right] .
$$

Desenvolvendo cada parcela da última expressão

$$
\begin{aligned}
E\left[1(T>t) 1\left(T_{(i)} \leq t<T_{(i+1)}\right) \mid \mathfrak{F}_{t}\right] & =E\left[\int_{0}^{\infty} 1(s>t) d N_{\Phi}(s) 1\left(T_{(i)} \leq t<T_{(i+1)}\right) \mid \mathfrak{F}_{t}\right] \\
& =E\left[\int_{0}^{\infty} 1(s>t) d A_{\Phi}(s) 1\left(T_{(i)} \leq t<T_{(i+1)}\right) \mid \mathfrak{F}_{t}\right]
\end{aligned}
$$

onde a segunda igualdade é devido ao lema A.2.2. Portanto

$$
\begin{gathered}
E\left[\int_{0}^{\infty} 1(s>t) d A_{\Phi}(s) 1\left(T_{(i)} \leq t<T_{(i+1)}\right) \mid \mathfrak{F}_{t}\right]= \\
=\sum_{k=1}^{n} \sum_{j=1}^{n} E\left[\int_{0}^{\infty} 1(s>t) 1\left(Y_{(k) j}<s \leq T\right) d A_{(k) j}(s) 1\left(T_{(i)} \leq t<T_{(i+1)}\right) \mid \mathfrak{F}_{t}\right]
\end{gathered}
$$




$$
\begin{aligned}
& =\sum_{k=1}^{n} \sum_{j=1}^{n} E\left[\int_{0}^{\infty} 1(s>t) 1\left(Y_{(k) j}<s \leq T\right) d N_{(k) j}(s) 1\left(T_{(i)} \leq t<T_{(i+1)}\right) \mid \mathfrak{F}_{t}\right] \\
& =\sum_{k=1}^{n} \sum_{j=1}^{n} E\left[1\left(T_{(k) j}>t\right) 1\left(Y_{(k) j}<T_{(k) j} \leq T\right) 1\left(T_{(i)} \leq t<T_{(i+1)}\right) \mid \mathfrak{F}_{t}\right] \\
& =\sum_{k=i+1}^{n} \sum_{j=1}^{n} E\left[1\left(T_{(k) j}>t\right) 1\left(T=T_{(k) j}\right) 1\left(T_{(i)} \leq t<T_{(i+1)}\right) \mid \mathfrak{F}_{t}\right],
\end{aligned}
$$

onde a primeira igualdade é proveniente da decomposição do compensador $A_{\Phi}(t)$ (teorema 2.1.1). Se denotamos cada $E\left[1(T>t) 1\left(T_{(i)} \leq t<T_{(i+1)}\right) \mid \mathfrak{F}_{t}\right]$ por $h(i, t)$ temos que

$$
P\left(T>t \mid \mathfrak{F}_{t}\right)=\sum_{i=0}^{n} h(i, t) 1\left(T_{(i)} \leq t<T_{(i+1)}\right)
$$

o que demostra o teorema.

Como consequência desse lema, o seguinte teorema nos permite definir uma assinatura dinâmica associada a $P\left(T>t \mid \mathfrak{F}_{t}\right)$

Teorema 2.2.1 (Teorema de assinatura dinâmica para $\mathfrak{F}_{t}$ )

Seja $T$ o tempo de vida de um sistema coerente $\Phi$ de ordem $n$ e sejam $T_{1}, . ., T_{n}$ os $\mathfrak{F}_{t}$-tempos de parada representando os tempos de vida dos seus componentes com $T_{(0)}=0$ e $T_{(n+1)}=\infty$. Então, sob o evento $\{T>t\}$, temos:

$$
P\left(T>t \mid \mathfrak{F}_{t}\right)=\sum_{i=1}^{n} \beta(i, t) 1\left(T_{(i)}>t\right)
$$

onde

$$
\beta(i, t)=\sum_{j=1}^{n} P\left(T=T_{(i) j} \mid \mathfrak{F}_{t}\right) 1\left(T_{(i) j}>t\right)
$$

$\operatorname{com} \beta(i, t) \geq 0, \sum_{i=1}^{n} \beta(i, t)=1$.

\section{Demonstração}

Primeiro provaremos a decomposição para $P\left(T>t \mid \mathfrak{F}_{t}\right)$. Para tal usaremos o fato de

$$
1\left(T_{(i)} \leq t<T_{(i+1)}\right)=1\left(T_{(i)} \leq t\right)-1\left(T_{(i+1)} \leq t\right)
$$

na decomposição de $P\left(T>t \mid \mathfrak{F}_{t}\right)$ do lema 2.2.1. Assim

$$
P\left(T>t \mid \mathfrak{F}_{t}\right)=\sum_{i=0}^{n} h(i, t) 1\left(T_{(i)} \leq t<T_{(i+1)}\right)=\sum_{i=0}^{n} h(i, t)\left(1\left(T_{(i)} \leq t\right)-1\left(T_{(i+1)} \leq t\right)\right) .
$$

Multiplicando e rescrevendo o somatório temos 


$$
\begin{aligned}
P\left(T>t \mid \mathfrak{F}_{t}\right) & =\sum_{i=0}^{n} h(i, t)\left(1\left(T_{(i)} \leq t\right)-\sum_{i=1}^{n} h(i-1, t) 1\left(T_{(i)} \leq t\right)\right) \\
& =\sum_{i=0}^{n} h(i, t)\left(1\left(T_{(i)} \leq t\right)-1\left(T_{(i+1)} \leq t\right)\right) \\
& =h(0, t) 1\left(T_{(0)} \leq t\right)+\sum_{i=1}^{n}(h(i, t)-h(i-1, t)) 1\left(T_{(i)} \leq t\right) \\
& =h(0, t) 1\left(T_{(0)} \leq t\right)+\sum_{i=1}^{n}(h(i, t)-h(i-1, t))+\sum_{i=1}^{n}(h(i-1, t)-h(i, t)) 1\left(T_{(i)}>t\right) \\
& =\sum_{i=1}^{n}(h(i-1, t)-h(i, t)) 1\left(T_{(i)}>t\right) .
\end{aligned}
$$

A expressão $h(0, t) 1\left(T_{(0)} \leq t\right)+\sum_{i=1}^{n}(h(i, t)-h(i-1, t))$ é igual a zero porque

$$
h(0, t) 1\left(T_{(0)} \leq t\right)+\sum_{i=1}^{n}(h(i, t)-h(i-1, t))=h(0, t)+\sum_{i=1}^{n}(h(i, t)-h(i-1, t))=h(n, t)=0 .
$$

Portanto concluimos que

$$
P\left(T>t \mid \mathfrak{F}_{t}\right)=\sum_{i=1}^{n} \beta(i, t) 1\left(T_{(i)}>t\right)
$$

onde os $\beta(i, t)$ são iguais a os $h(i-1)-h(i)$ para $i=1, \ldots, n$.

Em seguida provaremos que os $\beta(i, t)$ são positivos. Basta notar que $\beta(i, t)=h(i-1, t)-h(i, t)=$ $1\left(T_{(i)}>t\right) P\left(T=T_{(i)} \mid \mathfrak{F}_{t}\right) \geq 0$.

Os $\beta(i, t)$ têm soma igual a um. Da definição de $\beta(i, t)$ temos

$$
\begin{aligned}
\sum_{i=1}^{n} \beta(i, t) & =\sum_{i=1}^{n} h(i-1, t)-h(i, t)=h(0, t) \\
& =\sum_{k=1}^{n} \sum_{j=1}^{n} 1\left(T_{(k) j}>t\right) P\left(T=T_{(k) j} \mid \mathfrak{F}_{t}\right) \\
& =\sum_{k=1}^{n} \sum_{j=1}^{n} P\left(T=T_{(k) j}, T_{(k) j}>t \mid \mathfrak{F}_{t}\right) \\
& =\sum_{k=1}^{n} \sum_{j=1}^{n} P\left(T=T_{(k) j}, T>t \mid \mathfrak{F}_{t}\right) \\
& =P\left(T \geq T_{(1)}, T>t \mid \mathfrak{F}_{t}\right)
\end{aligned}
$$


mas $P\left(T \geq T_{(1)}, T>t \mid \mathfrak{F}_{t}\right)=1$ no evento $\{T>t\}$ terminando a demonstração do teorema. A terceira igualdade segue do fato que $1\left(T_{(k) j}>t\right)$ é $\mathfrak{F}_{t}$-mensuravel.

Definição 2.2.1 $O$ vetor $\beta(t)$ com coordenadas $\beta(i, t), i=1, . ., n$ é chamado vetor de assinatura dinâmica com respeito a $\mathfrak{F}_{t}$.

Sob as hipóteses que os tempos de vidas são independentes e identicamente distribuidos a assinatura dinâmica $\beta(t)$ se torna basicamente a assinatura $\alpha$.

Teorema 2.2.2 ( Teorema de reconstrução da assinatura clássica)

Seja $T$ o tempo de vida de um sistema coerente $\Phi$ de ordem $n$ tal que os tempos de vida $T_{1}, . ., T_{n}$ dos componentes são $\mathfrak{F}_{t}$-tempos de parada independentes e identicamente distribuidos com função de distribução $F$ contínua. Então no nível de informação $\mathfrak{F}_{t}$, no evento $\{T>t\}$, temos que:

$$
P\left(T>t \mid \mathfrak{F}_{t}\right)=\sum_{k=1}^{n} \alpha_{k} 1\left(T_{(k)}>t\right)
$$

onde $\alpha_{k}$ são as coordenadas do vetor de assinatura $\alpha$.

\section{Demonstração}

A partir da decomposição básica de $P\left(T>t \mid \mathfrak{F}_{t}\right)$ será suficiente analisar os $\beta(k, t)$

$$
\begin{aligned}
\beta(k, t) & =h(k-1, t)-h(k, t) \\
& =1\left(T_{(k)}>t\right) P\left(T=T_{(k)} \mid \mathfrak{F}_{t}\right) \\
& =\sum_{i=0}^{k-1} 1\left(T_{(i+1)}>t \geq T_{(i)}\right) P\left(T=T_{(k)} \mid \mathfrak{F}_{t}\right) \\
& =\sum_{i=0}^{k-1} P\left(T=T_{(k)}, T_{(i+1)}>t \geq T_{(i)} \mid \mathfrak{F}_{t}\right) \\
& =\sum_{i=0}^{k-1} P\left(T=T_{(k)}, T_{(i+1)}>t \geq T_{(i)} \mid \mathfrak{F}_{T_{(i)}}\right)
\end{aligned}
$$

onde a terceira igualdade segue porque $1\left(T_{(k) j}>t\right)$ é $\mathfrak{F}_{t}$-mensuravel e a última é devido ao lema 2.1.1. Como os tempos de vida dos componentes são independentes e identicamente distribuidos temos que para $k>i$ que:

$$
P\left(T=T_{(k)}, T_{(i+1)}>t \geq T_{(i)} \mid \mathfrak{F}_{T_{(i)}}\right)=P\left(T=T_{(k)}\right) P\left(T_{(i+1)}>t \geq T_{(i)} \mid \mathfrak{F}_{T_{(i)}}\right)
$$

e

$$
\beta(k, t)=\sum_{i=0}^{k-1} P\left(T=T_{(k)}, T_{(i+1)}>t \geq T_{(i)} \mid \mathfrak{F}_{T_{(i)}}\right)
$$




$$
\begin{aligned}
& =\sum_{i=0}^{k-1} P\left(T=T_{(k)}\right) P\left(T_{(i+1)}>t \geq T_{(i)} \mid \mathfrak{F}_{T_{(i)}}\right) \\
& =P\left(T=T_{(k)}\right) \sum_{i=0}^{k-1} P\left(T_{(i+1)}>t \geq T_{(i)} \mid \mathfrak{F}_{T_{(i)}}\right) \\
& =P\left(T=T_{(k)}\right) \sum_{i=0}^{k-1} P\left(T_{(i+1)}>t \geq T_{(i)} \mid \mathfrak{F}_{t}\right) \\
& =P\left(T=T_{(k)}\right) 1\left(T_{(k)}>t\right)
\end{aligned}
$$

onde a quarta igualdade é proveniente do lema 2.1.1.

Portanto, provamos que cada $\beta(k, t)=P\left(T=T_{(k)}\right) 1\left(T_{(k)}>t\right)$ e que

$$
P\left(T>t \mid \mathfrak{F}_{t}\right)=\sum_{k=1}^{n} \beta(k, t) 1\left(T_{(k)}>t\right)=\sum_{k=1}^{n} \alpha_{k} 1\left(T_{(k)}>t\right)
$$

o que conclui demonstração.

\section{Observação 2}

Tomando esperança na expressão de $P\left(T>t \mid \mathfrak{F}_{t}\right)$ temos $P(T>t)=\sum_{k=1}^{n} \alpha_{k} P\left(T_{(k)}>t\right)$ tal como esperavamos. 


\subsection{Assinatura Dinâmica ao nível de Informação Completa $\mathfrak{F}^{*}$}

Na seção anterior a evolução do sistema dinâmico foi analisado a partir das $\sigma$-álgebras $\left(\mathfrak{F}_{t}, t \geq 0\right)$. Nesta seção, além da informação fornecida pelas $\sigma$-álgebras $\left(\mathfrak{F}_{t}, t \geq 0\right)$, o observador do sistema coerente sabe se o evento $\{T \leq t\}$ ocorreu, ou não, a cada instante de inspeção $t$. Este tipo de observação é representado através das sub- $\sigma$-álgebras

$$
\mathcal{R}_{t}=\sigma\{1(T>s): 0<s \leq t\}
$$

Portanto a informação considerada nessa seção é a informação conjunta gerada pelas $\sigma$-álgebras $\left(\mathfrak{F}_{t}, t \geq 0\right)$ e $\left(\mathcal{R}_{t}, t \geq 0\right)$ que será denotada por $\left(\mathfrak{F}_{t}^{*}, t \geq 0\right)$ onde $\mathfrak{F}_{t}^{*}$ é a $\sigma$-álgebra mínimal, que contem $\mathfrak{F}_{t}$ e $\mathcal{R}_{t}$, isto é:

$$
\mathfrak{F}_{t}^{*}=\mathcal{R}_{t} \vee \mathfrak{F}_{t}
$$

Analisemos a evolução do sistema com respeito a $\mathfrak{F}_{t}^{*}$.

Lema 2.3.1 Seja $T$ o tempo de vida de um sistema coerente $\Phi$ de ordem $n$ tal que os tempos de vida $T_{1}, . ., T_{n}$ dos componentes são $\mathfrak{F}_{t}$-tempos de parada. Então:

$$
E\left[1(T>t) 1\left(T_{(i)} \leq t<T_{(i+1)}\right) \mid \mathfrak{F}_{t}^{*}\right]=\frac{1(T>t)}{P\left(T>t \mid \mathfrak{F}_{t}\right)} E\left[1(T>t) 1\left(T_{(i)} \leq t<T_{(i+1)}\right) \mid \mathfrak{F}_{t}\right]
$$

\section{Demonstração}

Calcularemos a esperança condicional do lado direito e provaremos que é igual à do lado esquerdo. Para isto usaremos que a $\sigma$-álgebra $\mathfrak{F}_{t}^{*}$ é gerada pelas interseções $\{T>s\} \cap B$ onde $\{T>s\} \in \mathcal{R}_{t}$ com $s \leq t$ e $B \in \mathfrak{F}_{t}$. Para simplificar a notacão denotaremos o evento $\left\{T_{(i)} \leq t<T_{(i+1)}\right\}$ por $I_{i}$. Calculando

$$
\begin{aligned}
\int 1(B) 1(T>s) E\left[1(T>t) 1\left(I_{i}\right) \mid \mathfrak{F}_{t}^{*}\right] d P & =\int 1(B) 1(T>t) 1\left(I_{i}\right) \frac{P\left(T>t \mid \mathfrak{F}_{t}\right)}{P\left(T>t \mid \mathfrak{F}_{t}\right)} d P \\
& =\int 1(B) P\left(T>t \mid \mathfrak{F}_{t}\right) \frac{E\left[1(T>t) 1\left(I_{i}\right) \mid \mathfrak{F}_{t}\right]}{P\left(T>t \mid \mathfrak{F}_{t}\right)} d P \\
& =\int 1(B) 1(T>t) \frac{E\left[1(T>t) 1\left(I_{i}\right) \mid \mathfrak{F}_{t}\right]}{P\left(T>t \mid \mathfrak{F}_{t}\right)} d P \\
& =\int 1(B) 1(T>s) 1(T>t) \frac{E\left[1(T>t) 1\left(I_{i}\right) \mid \mathfrak{F}_{t}\right]}{P\left(T>t \mid \mathfrak{F}_{t}\right)} d P
\end{aligned}
$$

onde a terceira igualdade é devido à definição de esperança condicional para $P\left(T>t \mid \mathfrak{F}_{t}\right)$. Assim a igualdade (2.9) vale para todo evento da forma $1(B) 1(T>s)$, os quais geram a $\sigma$-álgebra $\mathfrak{F}_{t}^{*}$ e portanto

$$
E\left[1(T>t) 1\left(I_{i}\right) \mid \mathfrak{F}_{t}^{*}\right]=\frac{1(T>t)}{P\left(T>t \mid \mathfrak{F}_{t}\right)} E\left[1(T>t) 1\left(I_{i}\right) \mid \mathfrak{F}_{t}\right]
$$


terminando a demonstração.

A análise da evolução de $P\left(T>t \mid \mathfrak{F}_{t}^{*}\right)$ do sistema coerente $(\Phi, \mathcal{C})$, será sob a hipótese de que o sistema está funcionando. Heuristicamente $P\left(T>t \mid \mathfrak{F}_{t}^{*}\right)$ evolui da seguinte forma: Se o instante de inspeção $t$ é antes da falha de qualquer componente, $P\left(T>t \mid \mathfrak{F}_{t}^{*}\right)=1$. Se no instante de inspeção $t$ há exatamente $i$ falhas ( i.e $\left\{T_{(i)} \leq t<T_{(i+1)}\right\}$ ) a probabilidade de falhar em um tempo posterior a $t$ será distribuida, basicamente, entre as possíveis falhas posteriores devido aos componentes $j$, $\left\{T=T_{(k) j}\right\}$, condicionado a $\left\{T \geq T_{(i+1)}\right\}$, isto é, com probabilidade $P\left(T=T_{(k) j}\right) / P\left(T \geq T_{(i+1)}\right)$ para $k \geq i+1$.

O lema que segue confirma tais detalhes.

Lema 2.3.2 ( Decomposição de $P\left(T>t \mid \mathfrak{F}_{t}^{*}\right.$ ) ao nível de informação $\mathfrak{F}_{t}^{*}$ )

Seja $T$ o tempo de vida de um sistema coerente $\Phi$ de ordem $n$ e sejam $T_{1}, . ., T_{n}$ os $\mathfrak{F}_{t}$-tempos de parada representando os tempos de vida dos seus componentes com $T_{(0)}=0$ e $T_{(n+1)}=\infty$. Então, a um nível de informação completa $\mathfrak{F}_{t}^{*}$, no evento $\{T>t\}$, temos que:

$$
P\left(T>t \mid \mathfrak{F}_{t}^{*}\right)=\sum_{i=0}^{n} h^{*}(i, t) 1\left(T_{(i)} \leq t<T_{(i+1)}\right)
$$

onde

$$
h^{*}(i, t)=\sum_{k=i+1}^{n} \sum_{j=1}^{n} 1\left(T \geq T_{(i+1)}\right) \frac{P\left(T=T_{(k) j}, T_{(i)} \leq t<T_{(i+1)} \mid \mathfrak{F}_{t}\right)}{P\left(T \geq T_{(i+1)}, T_{(i)} \leq t<T_{(i+1)} \mid \mathfrak{F}_{t}\right)} 1\left(T_{(k) j}>t\right)
$$

para $i=1, . ., n-1$ e $h(n)=0$ e onde $\left\{T_{(i) j}=s\right\}$ representa o evento $\left\{T_{(i)}=s, X_{i}=j\right\}$ (analogamente para $\left\{T_{(i) j}>s\right\}$ ou $\left.\left\{T_{(i) j}<s\right\}\right)$.

\section{Demonstração}

Como as falhas não ocorrem simultaneamente ${ }^{1}$, escrevemos

$$
1(T>t)=\sum_{i=0}^{n} 1(T>t) 1\left(T_{(i)} \leq t<T_{(i+1)}\right)
$$

Para calcular $P\left(T>t \mid \mathfrak{F}_{t}^{*}\right)=E\left[1(T>t) \mid \mathfrak{F}_{t}^{*}\right]$ calculemos cada uma das parcelas $E[1(T>$ $\left.t) 1\left(T_{(i)} \leq t<T_{(i+1)}\right) \mid \mathfrak{F}_{t}^{*}\right]$

Pelo lema 2.3.1

$E\left[\int_{0}^{\infty} 1(s>t) d N_{\Phi}(s) 1\left(T_{(i)} \leq t<T_{(i+1)}\right) \mid \mathfrak{F}_{t}^{*}\right]=\frac{1(T>t)}{P\left(T>t \mid \mathfrak{F}_{t}\right)} E\left[\int_{0}^{\infty} 1(s>t) d N_{\Phi}(s) 1\left(T_{(i)} \leq t<T_{(i+1)}\right) \mid \mathfrak{F}_{t}\right]$.

Como $1\left(T_{(i)} \leq t<T_{(i+1)}\right)$ é $\mathfrak{F}_{t}$-mensurável temos

\footnotetext{
${ }^{1} \mathrm{O}$ fato que as falhas não ocorrem simultaneamente implica que a falha do sistema deve coincidir com um único tempo $T_{(i) j}$ para algum $i, 1 \leq i, j \leq n$
} 


$$
\begin{gathered}
E\left[\int_{0}^{\infty} 1(s>t) d N_{\Phi}(s) 1\left(T_{(i)} \leq t<T_{(i+1)}\right) \mid \mathfrak{F}_{t}^{*}\right]=\frac{1(T>t) 1\left(T_{(i)} \leq t<T_{(i+1)}\right)}{P\left(T>t, T_{(i)} \leq t<T_{(i+1)} \mid \mathfrak{F}_{t}\right)} E\left[\int_{0}^{\infty} 1(s>t) d A_{\Phi}(s) \mid \mathfrak{F}_{t}\right] \\
\quad=\sum_{k, j=1}^{n} \frac{1(T>t)}{P\left(T>t, T_{(i)} \leq t<T_{(i+1)} \mid \mathfrak{F}_{t}\right)} E\left[\int_{0}^{\infty} 1(s>t) 1\left(Y_{(k) j}<s \leq T\right) d A_{(k) j}(s) 1\left(T_{(i)} \leq t<T_{(i+1)}\right) \mid \mathfrak{F}_{t}\right] \\
=\sum_{k, j=1}^{n} \frac{1(T>t) 1\left(T_{(i)} \leq t<T_{(i+1)}\right)}{P\left(T>t, T_{(i)} \leq t<T_{(i+1)} \mid \mathfrak{F}_{t}\right)} E\left[\int_{0}^{\infty} 1(s>t) 1\left(Y_{(k) j}<s \leq T\right) d N_{(k) j}(s) 1\left(T_{(i)} \leq t<T_{(i+1)}\right) \mid \mathfrak{F}_{t}\right] \\
\quad=\sum_{k=i+1}^{n} \sum_{j=1}^{n} \frac{1\left(T \geq T_{(i+1)}\right)}{P\left(T \geq T_{(i+1)}, T_{(i)} \leq t<T_{(i+1)} \mid \mathfrak{F}_{t}\right)} E\left[1\left(T_{(k) j}>t\right) 1\left(T=T_{(k) j}\right) 1\left(T_{(i)} \leq t<T_{(i+1)}\right) \mid \mathfrak{F}_{t}\right]
\end{gathered}
$$

onde a segunda igualdade é proveniente da decomposição do $\mathfrak{F}_{t}$-compensador $A_{\Phi}$ (Teorema 2.1.1) e a terceira igualdade segue da definição de $\mathfrak{F}_{t}$-compensador para cada $N_{(k) j}$.

Denotando por $h^{*}(i, t)$ a última expressão de $E\left[1(T>t) 1\left(T_{(i)} \leq t<T_{(i+1)}\right) \mid \mathfrak{F}_{t}^{*}\right]$ concluimos que

$$
P\left(T>t \mid \mathfrak{F}_{t}^{*}\right)=\sum_{i=0}^{n} h^{*}(i, t) 1\left(T_{(i)} \leq t<T_{(i+1)}\right)
$$

terminando a demonstração do lema.

Como consequência temos o seguinte resultado. Observe que $I_{k}=\left\{T_{(k)} \leq t<T_{(k+1)}\right\}$.

Teorema 2.3.1 (Teorema de assinatura dinâmica completa)

Seja $T$ o tempo de vida de um sistema coerente $\Phi$ de ordem $n$ e sejam $T_{1}, . ., T_{n}$ os $\mathfrak{F}_{t}$-tempos de parada representando os tempos de vida dos seus componentes com $T_{(0)}=0$ e $T_{(n+1)}=\infty$. Então, sob o evento $\{T>t\}$, temos:

$$
P\left(T>t \mid \mathfrak{F}_{t}^{*}\right)=\sum_{i=1}^{n} \beta^{*}(i, t) 1\left(T_{(i)}>t\right)
$$

onde os $\beta^{*}(i, t)$ são tais que:

a) No intervalo $I_{i-1}, \beta^{*}(i, t)$ é positivo.

b) No intervalo $I_{i}, \sum_{k=i+1}^{n} \beta^{*}(k, t)=1$ e cada $\beta^{*}(k, t)$ é não negativo para $k \geq i+1$.

$\operatorname{com} T_{(0)}=0, T_{(n+1)}=\infty$.

\section{Demonstração}


Primeiro provaremos a decomposição de $P\left(T>t \mid \mathfrak{F}_{t}^{*}\right)$. Para isso usaremos o fato de que

$$
1\left(T_{(i)} \leq t<T_{(i+1)}\right)=1\left(T_{(i)} \leq t\right)-1\left(T_{(i+1)} \leq t\right)
$$

Assim

$$
\begin{aligned}
P\left(T>t \mid \mathfrak{F}_{t}^{*}\right) & =\sum_{i=0}^{n} h^{*}(i, t) 1\left(T_{(i)} \leq t<T_{(i+1)}\right) \\
& =\sum_{i=0}^{n} h^{*}(i, t)\left(1\left(T_{(i)} \leq t\right)-1\left(T_{(i+1)} \leq t\right)\right) \\
& =\sum_{i=1}^{n}\left(h^{*}(i-1, t)-h^{*}(i, t)\right) 1\left(T_{(i)}>t\right) .
\end{aligned}
$$

Definindo $\beta^{*}(i, t)=h^{*}(i-1, t)-h^{*}(i, t)$, demonstramos a decomposição para $P\left(T>t \mid \mathfrak{F}_{t}^{*}\right)$.

Vejamos que os $\beta^{*}(i, t)$ satisfazem os item[a)] e o item[b)].

item[a)] Como cada $h^{*}(i, t)$ só é significativo no intervalo $I_{i}$, temos que

$$
\beta^{*}(i, t)=h^{*}(i-1, t) 1\left(I_{i-1}\right)-h^{*}(i, t) 1\left(I_{i}\right) .
$$

Assim, no intervalo $I_{i-1}, \beta^{*}(i, t)$ é positivo, no intervalo $I_{i}, \beta^{*}(i, t)$ é negativo e zero em qualquer outro intervalo.

item[b)] No intervalo $I_{i}$ temos

$$
\begin{aligned}
\sum_{k=i+1}^{n} \beta^{*}(k, t) & =\sum_{k=i+1}^{n}\left(h^{*}(k-1, t) 1\left(I_{k-1}\right)-h^{*}(k, t) 1\left(I_{k}\right)\right) \\
& =h^{*}(i, t) 1\left(I_{i}\right)-h^{*}(n, t) 1\left(I_{n}\right) \\
& =h^{*}(i, t) 1\left(I_{i}\right)-0 \\
& =1\left(T \geq T_{(i+1)}\right) 1\left(I_{i}\right)=1
\end{aligned}
$$

no evento $1(T>t) 1\left(I_{i}\right)$, e concluimos a demonstração do teorema. 
Com o teorema anterior podemos definir.

Definição 2.3.1 O vetor $\beta^{*}(t)$ com coordenadas $\beta^{*}(i, t), i=1, . ., n$ é chamado vetor de assinatura dinâmica com respeito $a \mathfrak{F}_{t}^{*}$.

Sob as condições usuais de que os tempos de vida dos componentes são independentes e identicamente distribuidos, recuperamos a noção de assinatura dinâmica de Samaniego a partir da assinatura dinâmica com respeito a $\mathfrak{F}_{t}^{*}$.

Teorema 2.3.2 (Teorema de reconstrução)

Seja $T$ o tempo de vida de um sistema coerente $\Phi$ de ordem $n$ tal que os tempos de vida $T_{1}, . ., T_{n}$ dos componentes são $\mathfrak{F}_{t}$-tempos de parada independentes e identicamente distribuidos com função de distribução contínua $F$. Então, ao nível de informação completa $\mathfrak{F}_{t}^{*}$, no evento $\{T>t\} \cap\left\{T_{(i)} \leq\right.$ $\left.t<T_{(i+1)}\right\}$, temos que:

$$
P\left(T>t \mid \mathfrak{F}_{t}^{*}\right)=\sum_{k=i+1}^{n} \beta_{k} 1\left(T_{(k)}>t\right)
$$

onde $\beta_{k}$ são as coordenadas do vetor de assinatura dinâmica dado o evento $\{T>t\} \cap\left\{T_{(i)} \leq t<\right.$ $\left.T_{(i+1)}\right\}$

\section{Demonstração}

No evento $\left\{T_{(i)} \leq t<T_{(i+1)}\right\}$ analisaremos $P\left(T>t \mid \mathfrak{F}_{t}^{*}\right)$. Usando o teorema da assinatura temos

$$
\begin{aligned}
P\left(T>t \mid \mathfrak{F}_{t}^{*}\right) & =\sum_{k=1}^{n} \beta^{*}(k, t) 1\left(T_{(k)}>t\right) 1\left(I_{i}\right) \\
& =\sum_{k=1}^{n}\left(h^{*}(k-1, t) 1\left(I_{k-1}\right)-h^{*}(k, t) 1\left(I_{(k)}\right)\right) 1\left(T_{(k)}>t\right) 1\left(I_{i}\right) \\
& =\sum_{k=i+1}^{n}\left(h^{*}(k-1, t) 1\left(I_{k-1}\right)-h^{*}(k, t) 1\left(I_{(k)}\right)\right) 1\left(T_{(k)}>t\right) 1\left(I_{i}\right) \\
& =h^{*}(i, t) 1\left(I_{i}\right) 1\left(T_{(i+1)}>t\right)
\end{aligned}
$$

onde a terceira igualdade vem do fato que $1\left(T_{(k)}>t\right) 1\left(I_{i}\right)=1$ se $k>i$. Resta analisar $h^{*}(i, t) 1\left(I_{i}\right) 1\left(T_{(i+1)}>t\right)$.

Assim

$$
\begin{aligned}
h^{*}(i, t) & =\sum_{k=i+1}^{n} \sum_{j=1}^{n} 1\left(T \geq T_{(i+1)}\right) \frac{P\left(T=T_{(k) j}, T_{(i)} \leq t<T_{(i+1)} \mid \mathfrak{F}_{t}\right)}{P\left(T \geq T_{(i+1)}, T_{(i)} \leq t<T_{(i+1)} \mid \mathfrak{F}_{t}\right)} 1\left(T_{(k) j}>t\right) \\
& =\sum_{k=i+1}^{n} \sum_{j=1}^{n} 1\left(T \geq T_{(i+1)}\right) \frac{P\left(T=T_{(k) j}, T_{(i)} \leq t<T_{(i+1)}, T_{(k) j}>t \mid \mathfrak{F}_{t}\right)}{P\left(T \geq T_{(i+1)}, T_{(i)} \leq t<T_{(i+1)} \mid \mathfrak{F}_{t}\right)} \\
& =\sum_{k=i+1}^{n} \sum_{j=1}^{n} 1\left(T \geq T_{(i+1)}\right) \frac{P\left(T=T_{(k) j}, T_{(i)} \leq t<T_{(i+1)}, T>t \mid \mathfrak{F}_{t}\right)}{P\left(T \geq T_{(i+1)}, T_{(i)} \leq t<T_{(i+1)} \mid \mathfrak{F}_{t}\right)}
\end{aligned}
$$




$$
\begin{aligned}
& =\sum_{k=i+1}^{n} 1\left(T \geq T_{(i+1)}\right) \frac{P\left(T=T_{(k)}, T_{(i)} \leq t<T_{(i+1)} \mid \mathfrak{F}_{t}\right)}{P\left(T \geq T_{(i+1)}, T_{(i)} \leq t<T_{(i+1)} \mid \mathfrak{F}_{t}\right)} 1\left(T_{(k)}>t\right) \\
& =\sum_{k=i+1}^{n} 1\left(T \geq T_{(i+1)}\right) \frac{P\left(T=T_{(k)}, T_{(i)} \leq t<T_{(i+1)} \mid \mathfrak{F}_{T_{i}}\right)}{P\left(T \geq T_{(i+1)}, T_{(i)} \leq t<T_{(i+1)} \mid \mathfrak{F}_{T_{i}}\right)} 1\left(T_{(k)}>t\right) .
\end{aligned}
$$

A última linha é consequência direta do lema 2.1.1. Como os tempos de vida dos componentes são independentes e identicamente distribuidos, para $k \geq i+1$

$$
\begin{gathered}
P\left(T=T_{(k)}, T_{(i)} \leq t<T_{(i+1)} \mid \mathfrak{F}_{T_{i}}\right)=P\left(T=T_{(k)}\right) P\left(T_{(i)} \leq t<T_{(i+1)} \mid \mathfrak{F}_{T_{i}}\right) \\
P\left(T \geq T_{(i+1)}, T_{(i)} \leq t<T_{(i+1)} \mid \mathfrak{F}_{T_{i}}\right)=P\left(T \geq T_{(i+1)}\right) P\left(T_{(i)} \leq t<T_{(i+1)} \mid \mathfrak{F}_{T_{i}}\right)
\end{gathered}
$$

e

$$
\begin{aligned}
h^{*}(i, t) & =\sum_{k=i+1}^{n} 1\left(T \geq T_{(i+1)}\right) \frac{P\left(T=T_{(k)}, T_{(i)} \leq t<T_{(i+1)} \mid \mathfrak{F}_{T_{i}}\right)}{P\left(T \geq T_{(i+1)}, T_{(i)} \leq t<T_{(i+1)} \mid \mathfrak{F}_{T_{i}}\right)} 1\left(T_{(k)}>t\right) \\
& =\sum_{k=i+1}^{n} 1\left(T \geq T_{(i+1)}\right) \frac{P\left(T=T_{(k)}\right) P\left(T_{(i)} \leq t<T_{(i+1)} \mid \mathfrak{F}_{T_{i}}\right)}{P\left(T \geq T_{(i+1)}\right) P\left(T_{(i)} \leq t<T_{(i+1)} \mid \mathfrak{F}_{T_{i}}\right)} 1\left(T_{(k)}>t\right) \\
& =\sum_{k=i+1}^{n} 1\left(T \geq T_{(i+1)}\right) \frac{P\left(T=T_{(k)}\right)}{P\left(T \geq T_{(i+1)}\right)} 1\left(T_{(k)}>t\right) .
\end{aligned}
$$

Portanto, no evento $\left\{T_{(i)} \leq t<T_{(i+1)}\right\}$

$$
\begin{aligned}
P\left(T>t \mid \mathfrak{F}_{t}^{*}\right) & =h^{*}(i) 1\left(I_{i}\right) 1\left(T_{(i+1)}>t\right) \\
& =\left(\sum_{k=i+1}^{n} 1\left(T \geq T_{(i+1)}\right) \frac{P\left(T=T_{(k)}\right)}{P\left(T \geq T_{(i+1)}\right)} 1\left(T_{(k)}>t\right) 1\left(I_{i}\right)\right) 1\left(T_{(i+1)}>t\right) \\
& =\sum_{k=i+1}^{n} \frac{P\left(T=T_{(k)}\right)}{P\left(T \geq T_{(i+1)}\right)} 1\left(T_{(k)}>t\right) 1\left(I_{i}\right) 1\left(T \geq T_{(i+1)}\right) 1\left(T_{(i+1)}>t\right) \\
& =\sum_{k=i+1}^{n} \frac{P\left(T=T_{(k)}\right)}{P\left(T \geq T_{(i+1)}\right)} 1\left(T_{(k)}>t\right)
\end{aligned}
$$

o que demonstra o teorema.

Corolário 2.3.1 Seja T o tempo de vida de um sistema coerente $\Phi$ de ordem $n$ tal que os tempos de vida $T_{1}, . ., T_{n}$ dos componentes são $\mathfrak{F}_{t}$-tempos de parada independentes e identicamente distribuidos com função de distribução contínua $F$. Então, ao nível de informação completa $\mathfrak{F}_{t}^{*}$, no evento 
$\{T>t\} \cap\left\{T_{(i)} \leq t<T_{(i+1)}\right\}$, temos:

$$
P\left(T>t \mid \mathfrak{F}_{t}\right)=\sum_{k=i+1}^{n} \beta_{k} 1\left(T_{(k)}>t\right)
$$

onde $\beta_{k}$ são as coordenadas do vetor de assinatura dinâmica condicionado ao evento $\left\{T_{(i)} \leq t<\right.$ $\left.T_{(i+1)}\right\}$

\section{Demonstração}

Usando o lema 2.3.1 temos que $P\left(T>t, T_{(i)} \leq t<T_{(i+1)} \mid \mathfrak{F}_{t}^{*}\right)$

$$
\begin{aligned}
& =\frac{1(T>t)}{P\left(T>t, T_{(i)} \leq t<T_{(i+1)} \mid \mathfrak{F}_{t}\right)} E\left[1(T>t) 1\left(T_{(i)} \leq t<T_{(i+1)}\right) \mid \mathfrak{F}_{t}\right] \\
& =\frac{1\left(T \geq T_{(i+1)}\right)}{P\left(T \geq T_{(i+1)}, T_{(i)} \leq t<T_{(i+1)} \mid \mathfrak{F}_{t}\right)} E\left[1\left(T \geq T_{(i+1)}\right) 1\left(T_{(i)} \leq t<T_{(i+1)}\right) \mid \mathfrak{F}_{t}\right] \\
& =\frac{1\left(T \geq T_{(i+1)}\right)}{P\left(T \geq T_{(i+1)}, T_{(i)} \leq t<T_{(i+1)} \mid \mathfrak{F}_{\left.T_{(i)}\right)}\right.} E\left[1\left(T \geq T_{(i+1)}\right) 1\left(T_{(i)} \leq t<T_{(i+1)}\right) \mid \mathfrak{F}_{T_{(i)}}\right] \\
& =\frac{1\left(T \geq T_{(i+1)}\right)}{P\left(T \geq T_{(i+1)}\right) P\left(T_{(i)} \leq t<T_{(i+1)} \mid \mathfrak{F}_{T_{(i)}}\right)} E\left[1\left(T \geq T_{(i+1)}\right)\right] P\left(T_{(i)} \leq t<T_{(i+1)} \mid \mathfrak{F}_{T_{(i)}}\right) \\
& =E\left[1(T>t) 1\left(T_{(i)} \leq t<T_{(i+1)}\right) \mid \mathfrak{F}_{t}\right] .
\end{aligned}
$$

Portanto, usando o teorema anterior

$$
P\left(T>t \mid \mathfrak{F}_{t}\right)=\sum_{k=i+1}^{n} \beta_{k} 1\left(T_{(k)}>t\right)
$$

no evento $\{T>t\} \cap\left\{T_{(i)} \leq t<T_{(i+1)}\right\}$.

\section{Observação 3}

É possível recuperar a decomposição de $P\left(T>t \mid \mathfrak{F}_{t}\right)$ com respeito a $\mathfrak{F}_{t}$, usando $P\left(T>t \mid \mathfrak{F}_{t}^{*}\right)$. Para tal condicionamos, $P\left(T>t \mid \mathfrak{F}_{t}^{*}\right)=\sum_{i=0}^{n} h^{*}(i, t) 1\left(T_{(i)} \leq t<T_{(i+1)}\right)$ à $\sigma$-algebra $\mathfrak{F}_{t}$

Calculando $E\left[h^{*}(i, t) \mid \mathfrak{F}_{t}\right]$,

$$
\begin{aligned}
E\left[h^{*}(i, t) \mid \mathfrak{F}_{t}\right] & =\sum_{k=i+1}^{n} \sum_{j=1}^{n} P\left(T \geq T_{(i+1)} \mid \mathfrak{F}_{t}\right) \frac{P\left(T=T_{(k) j}, T_{(i)} \leq t<T_{(i+1)} \mid \mathfrak{F}_{t}\right)}{P\left(T \geq T_{(i+1)}, T_{(i)} \leq t<T_{(i+1)} \mid \mathfrak{F}_{t}\right)} 1\left(T_{(k) j}>t\right) \\
& =\sum_{k=i+1}^{n} \sum_{j=1}^{n} P\left(T \geq T_{(i+1)}, T_{(i)} \leq t<T_{(i+1)} \mid \mathfrak{F}_{t}\right) \frac{P\left(T=T_{(k) j}, T_{(i)} \leq t<T_{(i+1)} \mid \mathfrak{F}_{t}\right)}{P\left(T \geq T_{(i+1)}, T_{(i)} \leq t<T_{(i+1)} \mid \mathfrak{F}_{t}\right)} \\
& =\sum_{k=i+1}^{n} \sum_{j=1}^{n} P\left(T=T_{(k) j} \mid \mathfrak{F}_{t}\right)
\end{aligned}
$$

recuperamos a decomposição de $P\left(T>t \mid \mathfrak{F}_{t}\right)$ no intervalo $\left\{T_{(i)} \leq t<T_{(i+1)}\right\}$. 
Usando os mesmos métodos desenvolvidos nas seções anteriores, podemos analisar o tempo de vida restante de um sistema coerente dado a informação $\mathfrak{F}_{t}$ ou a informação $\mathfrak{F}_{t}^{*}$. Analisaremos primeiro com respeito a $\mathfrak{F}_{t}^{*}$. Assim para $x \geq 0$

$$
\begin{aligned}
P\left(T>t+x \mid \mathfrak{F}_{t}^{*}\right) & =\sum_{i=0}^{n} h d^{*}(i, t, x) 1\left(T_{(i)} \leq t<T_{(i+1)}\right) \\
P\left(T>t+x \mid \mathfrak{F}_{t}^{*}\right) & =\sum_{i=1}^{n} \beta d^{*}(i, t, x) 1\left(T_{(i)}>t\right)
\end{aligned}
$$

onde

$$
h d^{*}(i, t, x)=\sum_{k=i+1}^{n} \sum_{j=1}^{n} 1\left(T \geq T_{(i+1)}\right) \frac{P\left(T=T_{(k) j}, T_{(i)} \leq t<T_{(i+1)}, T_{(k) j}>t+x \mid \mathfrak{F}_{t}\right)}{P\left(T \geq T_{(i+1)}, T_{(i)} \leq t<T_{(i+1)} \mid \mathfrak{F}_{t}\right)}
$$

$\mathrm{e}$

$$
\beta d^{*}(i, t, x)=h d^{*}(i-1, t, x)-h d^{*}(i, t, x) .
$$

Se definimos $\beta d^{*}(t, x)$ como o vetor com coordenadas $\beta d^{*}(i, t, x)$, temos o seguinte resultado:

Teorema 2.3.3 Considere dois sistemas coerentes com tempos de vida $S_{1}$ e $S_{2}$, e com assinaturas dinâmicas $\beta_{1}^{*}(t)$ e $\beta_{2}^{*}(t)$ respectivamentes. Então:

a) Se $\beta_{1}^{*}(t) \leq_{s t} \beta_{2}^{*}(t)$ então no evento $\left\{S_{1}>t\right\} \cap\left\{S_{2}>t\right\}$

$$
P\left(S_{1}>t \mid \mathfrak{F}_{t}^{*}\right) \leq P\left(S_{2}>t \mid \mathfrak{F}_{t}^{*}\right)
$$

b) Se $\beta_{1} d^{*}(t, x) \leq{ }_{s t} \beta_{2} d^{*}(t, x)$ para todo $x \geq 0$ então no evento $\left\{S_{1}>t\right\} \cap\left\{S_{2}>t\right\}$

$$
\left(S_{1}-t \mid \mathfrak{F}_{t}^{*}\right) \leq_{s t}\left(S_{2}-t \mid \mathfrak{F}_{t}^{*}\right)
$$

\section{Demonstração}

Item[a)]. Se $T$ é o tempo de vida de um sistema coerente, do teorema de assinaturas temos

$$
\begin{aligned}
P\left(T>t \mid \mathfrak{F}_{t}^{*}\right) & =\sum_{i=1}^{n} \beta^{*}(i, t) 1\left(T_{(i)}>t\right) \\
& =\sum_{i=0}^{n}\left(\sum_{k=i+1}^{n} \beta^{*}(k, t)\right) 1\left(T_{(i)} \leq t<T_{(i+1)}\right)
\end{aligned}
$$

Então se $\beta_{1}^{*}(t) \leq_{s t} \beta_{2}^{*}(t)$ temos $P\left(S_{1}>t \mid \mathfrak{F}_{t}^{*}\right) \leq P\left(S_{2}>t \mid \mathfrak{F}_{t}^{*}\right)$.

Item $[\mathrm{b})]$. Basta notar que

$$
P\left(T>t+x \mid \mathfrak{F}_{t}^{*}\right)=\sum_{i=0}^{n} h d^{*}(i, t, x) 1\left(T_{(i)} \leq t<T_{(i+1)}\right)
$$




$$
=\sum_{i=0}^{n}\left(\sum_{j=i}^{n} \beta d^{*}(j, t, x)\right) 1\left(T_{(i)} \leq t<T_{(i+1)}\right)
$$

para qualquer sistema coerente, $\operatorname{assim} \beta_{1} d^{*}(t, x) \leq_{s t} \beta_{2} d^{*}(t, x)$ implica que

$$
P\left(S_{1}>t+x \mid \mathfrak{F}_{t}^{*}\right) \leq P\left(S_{2}>t+x \mid \mathfrak{F}_{t}^{*}\right)
$$

para $x \geq 0$, terminando a demonstração do teorema. 


\section{Capítulo 3}

\section{Aplicações}

\subsection{Importância de Barlow-Proschan e Assinatura}

Um dos objetivo das medidas de importância da confiabilidade dos componentes para a confiabilidade do sistema é identificar componentes cuja melhora implica na melhora do desempenho do sistema. Existem muitas medidas de importância da confiabilidade dos componentes para a confiabilidade do sistema, e entre as mais utilizadas está a importância de Barlow e Proschan [5]. Neste trabalho, Barlow e Proschan consideram que os componentes são independentes com função de distribuição contínua e definem

$$
I_{j}^{B P}=\int_{0}^{\infty}\left[P\left(T>t \mid T_{j}>t\right)-P\left(T>t \mid T_{j} \leq t\right)\right] d F_{j}(t)
$$

onde $F_{j}(t)$ é a função de distribuição continua do componente $j$. Em outro artigo, Bueno e Menezes [9], a importância de Barlow e Proschan é considerada para componentes dependentes e, possivelmente, com falhas simultâneas. No contexto das assinaturas o sistema é considerado como função das estatísticas de ordem ( não mais dos componentes) e mesmo que os componentes sejam independentes e identicamente distribuidos as estatística de ordem não o são. É interessante perguntar sobre a importância de confiabilidade do componente $j$ para que a falha do sistema coincida com a $i$-ésima falha dos componentes.

No artigo de Bueno e Menezes [9] temos a seguinte definição.

Definição 3.1.1 Seja $T$ o tempo de vida de um sistema coerente $\Phi$ de ordem n e $T_{j}$ o tempo de vida do componente $j$. A Importância de Barlow-Proschan do componente $j$ para a confiabilidade do sistema é definida por

$$
I_{j}^{B P}=E\left\{\left[A_{j}(T)-A_{j}\left(Y_{j}\right)\right]^{+}\right\}
$$

onde $A_{j}$ é o $\mathfrak{F}_{t}$-compensador de $N_{j}$ e $Y_{j}$ é o nível crítico do componente $j$, definido como o primeiro instante a partir do qual a falha do componente $j$ causa a falha do sistema.

\section{Observação 4}


Quando os tempos de vida dos componentes são independentes e totalmente inacessíveis ( Ver apêndice para a definição ) o $\mathfrak{F}_{t}$-compensador de $1\left(T_{j} \leq t\right)$ é $A_{j}=-\ln \left(\bar{F}_{j}\left(t \wedge T_{j}\right)\right)$ e portanto a importãncia de Barlow-Proschan do componente $j$ para a confiabilidade do sistema é dada por

$$
I_{j}^{B P}=E\left\{\left[-\ln \frac{\bar{F}_{j}\left(T \wedge T_{j}\right)}{\bar{F}_{j}\left(Y_{j} \wedge T_{j}\right)}\right]^{+}\right\}
$$

Definição 3.1.2 Seja T o tempo de vida de um sistema coerente $\Phi$ de ordem $n$ com componentes identicamente distribuidos e seja $A_{(i) j}(t)$ o $\mathfrak{F}_{t}$-compensador do processo $N_{(i) j}(t)$. Então a importância da confiabilidade do componente $j$ para a confiabilidade do sistema ao nível $i$ (para que a falha do sistema coincida com a i-esima falha dos componentes) é:

$$
I_{(i) j}^{B P}=E\left\{\left[A_{(i) j}(T)-A_{(i) j}\left(Y_{(i) j}\right)\right]^{+}\right\}
$$

onde $a^{+}=\max \{a, 0\}$ e $Y_{(i) j}$ é o nivel crítico do componente $j$ para que a falha do sistema coincida com a i-ésima falha dos componentes.

A Importância da confiabilidade da i-falha para a confiabilidade do sistema será definida como:

$$
I_{(i)}^{B P}=\sum_{j=1}^{n} I_{(i) j}^{B P} .
$$

Observe que $\left\{T_{(i) j}=s\right\}$ expressa o evento $\left\{T_{(i)}=s\right\} \cap\left\{X_{i}=j\right\}$. Analogamente para $\left\{T_{(i) j} \geq s\right\}$, $\left\{T_{(i) j} \leq s\right\}$ e $F_{(i) j}(s)=P\left(T_{(i)} \leq s, X_{i}=j\right)$, uma sub-distribução que consideramos padronizada.

Proposição 3.1.1 Seja T o tempo de vida de um sistema coerente $\Phi$ de ordem $n$ com componentes independentes e identicamente distribuidas e seja $A_{(i) j}(t)$ o $\mathfrak{F}_{t}$-compensador do processo $N_{(i) j}(t) d a$ forma $A_{(i) j}(t)=-\ln \left(\bar{F}_{(i) j}\left(t \wedge T_{(i) j}\right)\right)$. Então a importância da confiabilidade do componente $j$ para a confiabilidade do sistema no nivel $i$ é:

$$
I_{(i) j}^{B P}=P\left(T=T_{(i) j}\right)
$$

\section{Demonstração}

Da definição de importância temos que

$$
I_{(i) j}^{B P}=E\left\{\left[A_{(i) j}(T)-A_{(i) j}\left(Y_{(i) j}\right)\right]^{+}\right\}=E\left[\int_{0}^{\infty} 1\left(Y_{(i) j}<s \leq T\right) d A_{(i) j}(s)\right]
$$

e do fato que $A_{(i) j}(t)=-\ln \left(\bar{F}_{(i) j}\left(t \wedge T_{(i) j}\right)\right)$, a importância da confiabilidade do componente $j$ para a confiabilidade do sistema na $i$-ésima falha, $I_{(i) j}^{B P}$, é igual a

$$
\begin{aligned}
I_{(i) j}^{B P} & =E\left[\int_{0}^{\infty} 1\left(Y_{(i) j}<s \leq T\right) d\left(-\ln \left(\bar{F}_{(i) j}\left(t \wedge T_{(i) j}\right)\right)\right)\right] \\
& =E\left[\int_{0}^{T_{(i) j}} 1\left(Y_{(i) j}<s \leq T\right) d\left(-\ln \left(\bar{F}_{(i) j}(s)\right)\right)\right]
\end{aligned}
$$




$$
\begin{aligned}
& =\int_{0}^{\infty} E\left[\int_{0}^{T_{(i) j}} 1\left(Y_{(i) j}<s \leq T\right) \frac{d F_{(i) j}(s)}{\bar{F}_{(i) j}(s)} \mid T_{(i) j}=t\right] d F_{(i) j}(t) \\
& =\int_{0}^{\infty} E\left[\int_{0}^{t} 1\left(Y_{(i) j}<s \leq T\right) \frac{d F_{(i) j}(s)}{\bar{F}_{(i) j}(s)} \mid T_{(i) j}=t\right] d F_{(i) j}(t) \\
& =\int_{0}^{\infty} E\left[1\left(Y_{(i) j}<T_{(i) j} \leq T\right) \mid T_{(i) j}=t\right] d F_{(i) j}(t) \\
& =\int_{0}^{\infty} P\left(T=T_{(i) j} \mid T_{(i) j}=t\right) d F_{(i) j}(t) .
\end{aligned}
$$

Assim

$$
I_{(i) j}^{B P}=\int_{0}^{\infty} P\left(T=T_{(i) j} \mid T_{(i) j}=t\right) d F_{(i) j}(t)=P\left(T=T_{(i) j}\right) .
$$

terminando a demonstração.

A hipótese que os componentes têm tempos de vida independentes e identicamente distribuidos, não é essencial para tal definição.

Proposição 3.1.2 Seja $T$ o tempo de vida de um sistema coerente $\Phi$ com tempos de vida dos componentes $T_{i}, i=1, . ., n \mathfrak{F}_{t^{-}}$tempos de parada totalmente inacessíveis, e seja $A_{(i) j}(t)$ o $\mathfrak{F}_{t^{-}}$ compensador do processo $N_{(i) j}(t)$. Então a importância da confiabilidade do componente $j$ para a confiabilidade do sistema no nivel $i$ é:

$$
I_{(i) j}^{B P}=\int_{0}^{\infty} P\left(T=T_{(i) j} \mid T_{(i) j}=s\right) d F_{(i) j}(s)
$$

\section{Demonstração}

Consideramos o processo $\mathfrak{F}_{t}$-previsível $1\left(Y_{(i) j}<t \leq T\right)$. Como o $\mathfrak{F}_{t}$-compensador $A_{(i) j}(t)$ é contínuo temos que

$$
\begin{aligned}
I_{(i) j}^{B P} & =E\left\{\left[A_{(i) j}(T)-A_{(i) j}\left(Y_{(i) j}\right)\right]^{+}\right\} \\
& =E\left\{\int_{0}^{\infty} 1\left(Y_{(i) j}<t \leq T\right) d A_{(i) j}(s)\right\} \\
& =E\left\{\int_{0}^{\infty} 1\left(Y_{(i) j}<t \leq T\right) d N_{(i) j}(s)\right\} \\
& =E\left[Y_{(i) j}<T_{(i) j} \leq T\right]=P\left(Y_{(i) j}<T_{(i) j} \leq T\right) \\
& =P\left(T=T_{(i) j}\right)=\int_{0}^{\infty} P\left(T=T_{(i) j} \mid T_{(i) j}=s\right) d F_{(i) j}(s)
\end{aligned}
$$

terminando a demonstração da proposição.

O seguinte teorema relaciona a importância de Barlow-Proschan com a assinatura do sistema coerente. 
Teorema 3.1.1 Seja $T$ o tempo de vida de um sistema coerente $\Phi$ de ordem $n$ tal que os tempos de vida $T_{1}, . ., T_{n}$ dos componentes são $\mathfrak{F}_{t}$-tempos de parada independentes e identicamente distribuidos com função de distribução $F$ contínua. A importância de confiabilidade do sistema no nível $i$ é:

$$
I_{(i)}^{B P}=\alpha_{i}
$$

onde $\alpha_{i}$ é o $i$-ésimo componente do vetor de assinatura clássica.

\section{Demonstração}

Como nosso sistema coerente $\Phi$ de ordem $n$ as falhas não ocorrem simultaneamente temos que para qualquer $i=1, . ., n$

$$
1(T=T(i))=\sum_{j=1}^{n} 1\left(T=T_{(i) j}\right),
$$

$\operatorname{assim}$

$$
\begin{aligned}
\alpha_{i} & =P\left(T=T_{(i)}\right)=\sum_{j=1}^{n} E\left[1\left(T=T_{(i) j}\right)\right] \\
& =\sum_{j=1}^{n} E\left[\int_{0}^{\infty} 1\left(Y_{(i) j}<s \leq T\right) d N_{(i) j}(s)\right] \\
& =\sum_{j=1}^{n} E\left[\int_{0}^{\infty} 1\left(Y_{(i) j}<s \leq T\right) d A_{(i) j}(s)\right] \\
& =\sum_{j=1}^{n} E\left[\int_{0}^{\infty} 1\left(Y_{(i) j}<s \leq T\right) d A_{(i) j}(s)\right] \\
& =\sum_{j=1}^{n} E\left\{\left[A_{(i) j}(T)-A_{(i) j}\left(Y_{(i) j}\right)\right]^{+}\right\} \\
& =\sum_{j=1}^{n} I_{(i) j}^{B P}=I_{(i)}^{B P}
\end{aligned}
$$

finalizando a demonstração do teorema. 


\section{Exemplo 3.1.1}

Seja $\mathbf{T}=\left(T_{1}, T_{2}, T_{3}\right)$ um vetor de tempos de vida independentes e identicamente distribuidos, e consideremos o sistema coerente com tempo de vida $T=T_{1} \wedge\left(T_{2} \vee T_{3}\right)$. Calculemos então o vetor $\alpha=\left(\alpha_{1}, \alpha_{2}, \alpha_{3}\right)$. Se

$$
\begin{aligned}
& T_{1}<T_{2}<T_{3} \quad \text { então } \quad T=T_{(1)} \\
& T_{1}<T_{3}<T_{2} \quad \text { então } T=T_{(1)} \\
& T_{2}<T_{1}<T_{3} \quad \text { então } \quad T=T_{(2)} \\
& T_{2}<T_{3}<T_{1} \quad \text { então } \quad T=T_{(2)} \\
& T_{3}<T_{1}<T_{2} \quad \text { então } T=T_{(2)} \\
& T_{3}<T_{2}<T_{1} \quad \text { então } \quad T=T_{(2)}
\end{aligned}
$$

assim $\alpha_{1}=P\left(T=T_{(1)}\right)=1 / 3, \alpha_{2}=P\left(T=T_{(2)}\right)=2 / 3$ e $\alpha_{3}=P\left(T=T_{(3)}\right)=0$ e a decomposição do tempo de vida mediante a assinatura é:

$$
P(T \leq t)=\frac{1}{3} P\left(T_{(1)} \leq t\right)+\frac{2}{3} P\left(T_{(2)} \leq t\right) .
$$

Primeiro notemos que os níveis críticos do sistema são:

a) Para a primeira falha:

$$
Y_{(1) 1}=0, Y_{(1) 2}=\infty, Y_{(1) 3}=\infty \text {; }
$$

b) Para a segunda falha:

$$
Y_{(2) 1}=T_{2} \wedge T_{3}, Y_{(2) 2}=T_{3}, Y_{(2) 3}=T_{2}
$$

e $Y_{(3) j}=\infty$ para qualquer $j$, pois quando dois componentes falham o sistema falha.

Calculemos agora os $I_{(i) j}^{B P} \operatorname{com} 1 \leq i, j \leq 3$

$$
\begin{aligned}
I_{(1) 1}^{B P} & =E\left\{\left[A_{(1) 1}(T)-A_{(1) 1}\left(Y_{(1) 1}\right)\right]^{+}\right\} \\
& =E\left[\int_{0}^{\infty} 1(0<s \leq T) d N_{(1) 1}(s)\right] \\
& =P\left(T_{(1) 1} \leq T\right) \\
& =P\left(T_{1}<T_{2} \vee T_{3}\right)=\int_{0}^{\infty} P\left(T_{2}>s, T_{3}>s\right) d F(s) \\
& =\int_{0}^{\infty}(1-F(s))^{2} d F(s)=\frac{1}{3}
\end{aligned}
$$

e para as outras importâncias associadas à primeira falha, $I_{(1) 2}^{B P}=I_{(1) 3}^{B P}=0$. Assim $I_{(1) 1}^{B P}=1 / 3=\alpha_{1}$. 


$$
\begin{aligned}
I_{(2) 1}^{B P} & =E\left\{\left[A_{(2) 1}(T)-A_{(2) 1}\left(Y_{(2) 1}\right)\right]^{+}\right\} \\
& =E\left[\int_{0}^{\infty} 1\left(T_{2} \wedge T_{3}<s \leq T\right) d N_{(2) 1}(s)\right] \\
& =P\left(T_{2} \wedge T_{3}<T_{(2) 1} \leq T\right) \\
& =P\left(T_{2} \wedge T_{3}<T_{1} \leq T_{2} \vee T_{3}\right) \\
& =\int_{0}^{\infty} P\left(T_{2} \wedge T_{3}<s \leq T_{2} \vee T_{3}\right) d F(s) \\
& =2 \int_{0}^{\infty} F(s)(1-F(s)) d F(s)=\frac{1}{3} ; \\
I_{(2) 2}^{B P} & =E\left\{\left[A_{(2) 2}(T)-A_{(2) 2}\left(Y_{(2) 2}\right)\right]^{+}\right\} \\
= & E\left[\int_{0}^{\infty} 1\left(T_{3}<s \leq T\right) d N_{(2) 2}(s)\right] \\
= & P\left(T_{3}<T_{(2) 2} \leq T\right)=P\left(T_{3}<T_{2} \leq T_{1}\right) \\
= & \int_{0}^{\infty} P\left(s<T_{2} \leq T_{1}\right) d F(s) \\
= & 2 \int_{0}^{\infty}\left[\int_{s}^{\infty}(1-F(t)) d F(t)\right] d F(s)=\frac{1}{6} ; \\
I_{(2) 3}^{B P} & =E\left\{\left[A_{(2) 3}(T)-A_{(2) 3}\left(Y_{(2) 3}\right)\right]^{+}\right\} \\
= & E\left[\int_{0}^{\infty} 1\left(T_{2}<s \leq T\right) d N_{(2) 3}(s)\right] \\
= & \int_{0}^{\infty} P\left(s<T_{2} \leq T_{1}\right) d F(s) \\
&
\end{aligned}
$$

$\operatorname{logo} I_{(2)}^{B P}=I_{(2) 1}^{B P}+I_{(2) 2}^{B P}+I_{(2) 3}^{B P}=2 / 3=\alpha_{2}$

Como $Y_{(3) j}=\infty$ para qualquer $j$ temos $I_{(3) 1}^{B P}=I_{(3) 2}^{B P}=I_{(3) 3}^{B P}=I_{(3)}^{B P}=0=\alpha_{3}$.

\subsection{Importância de Componentes e Transformação no Compensador}

Um fato interessante observado é que várias medidas da confiabilidade dos componentes para a confiabilidade do sistema pode ser obtida como a diferença entre os tempos de vida médios dos sistemas quando realizamos uma modificação na função de distribução do tempo de vida do componente. É bem conhecido que existe uma correspondência bijetiva entre os espaço das funções de 
distribuição dos $\mathfrak{F}_{t}$-tempos de parada e o espaço dos $\mathfrak{F}_{t}$-compensadores dada pela fórmula exponencial de Doleans (Ver Arjas [1]). Interpretaremos qualquer modificação de algum componente (i.e modifição da sua função de distribuição ) através de uma transformação do respectivo compensador. As transformações do $\mathfrak{F}_{t}$-compensador do processo que usaremos são da forma:

$$
B_{i}(t)=\int_{0}^{t} \mu_{i}(s) d A_{i}(s)
$$

onde $1 \leq i \leq n$ e $\left(\mu_{i}(s), s \geq 0\right)$ é um processo positivo, $\mathfrak{F}_{t}$-previsível. Notemos que se $\mu_{i}(s) \leq 1$ o risco do processo $\mu_{i}(s) d A_{i}(s)$ é menor que $d A_{i}(s)$ e entenderemos isso como uma melhora no componente $i$, como por exemplo, através de uma operação de redundância.

Para $B_{i}(t)$ ser o novo $\mathfrak{F}_{t}$-compensador do processo $N_{i}(t)=1\left(T_{i} \leq t\right)$ deveremos alterar a medida de probabilidade usando o teorema de Girsanov ( Ver teorema A.2.6 ).

No caso de um reparo mínimo a transformação do $\mathfrak{F}_{t}$-compensador é da forma ( Ver Bueno [8]):

$$
B_{i}(t)=\int_{0}^{t} \frac{A_{i}(s)}{1+A_{i}(s)} d A_{i}(s)=A_{i}(t)-\ln \left(1+A_{i}(t)\right)
$$

$\mathrm{e}$

$$
L_{t}\left(\mu_{i}\right)=\left[\frac{A_{i}\left(T_{i}\right)}{1+A_{i}(T i)}\right]^{N_{i}(t)}\left(1+A_{i}(t)\right)
$$

Usando o fato de que, quando o compensador é contínuo, as variáveis aleátorias $A_{j}\left(T_{j}\right), 1 \leq j \leq$ $n$, são independentes e identicamente distribuidas com distribuição exponencial de parâmetro 1 (Ver teorema A.2.7). Definimos uma nova medida de probabilidade $Q_{\mu_{i}}$ pelo teorema de Radon-Nykodin

$$
\frac{d Q_{\mu_{i}}}{d P}=L\left(\mu_{i}\right)=A_{i}\left(T_{i}\right)
$$

Neste ponto devemos responder como uma transformação realizada no $\mathfrak{F}_{t}$-compensador correspondente ao componente $k$, afeta os $\mathfrak{F}_{t}$-compensadores das estatísticas de ordem.

Lema 3.2.1 Seja $T_{k}$ o $\mathfrak{F}_{t}$-tempo de parada, totalmente inacessivel, representando o tempo de vida do componente $k$ e seja $A_{k}(t)$ o $\mathfrak{F}_{t}$-compensador de processo $N_{k}(t)=1\left(T_{k} \leq t\right)$. Consideremos a transformação, através do reparo mínimo, no compensador do componente $k$,

$$
B_{k}(t)=\int_{0}^{t} \frac{A_{k}(s)}{1+A_{k}(s)} d A_{k}(s)
$$

Então para $i, 1 \leq i \leq n$, o $\mathfrak{F}_{t}$-compensador da $i$-ésima falha $A_{(i)}(t)$ é transformado em :

$$
B_{(i)}(t)=\sum_{j=1}^{n} B_{(i) j}
$$


onde

$$
B_{(i) k}(t)=\int_{0}^{t} \frac{A_{(i) k}(s)}{1+A_{(i) k}(s)} d A_{(i) k}(s)
$$

para o componente $k$

$$
B_{(i) j}(t)=A_{(i) j}(t)
$$

$\operatorname{para} j \neq k$.

\section{Demonstração}

Primeiro notemos que o $\mathfrak{F}_{t}$-compensador do componente $j$ pode ser escrito da forma $A_{j}(t)=$ $\sum_{i=1}^{n} A_{(i) j}(t)$ e que cada $d A_{(i) j}(t)$ é nulo fora do intervalo $\left\{T_{(i-1)}<t \leq T_{(i)}\right\}$ :

$$
d A_{j}(t)=\sum_{i=1}^{n} 1\left(T_{(i-1)}<t \leq T_{(i)}\right) d A_{(i) j} .
$$

Assim no caso de um reparo mínimo no componente $k$ através do $\mathfrak{F}_{t}$-compensador temos:

$$
\begin{aligned}
B_{k}(t) & =\int_{0}^{t} \frac{A_{k}(s)}{1+A_{k}(s)} d A_{k}(s) \\
& =\sum_{i=1}^{n} \int_{T_{(i-1)}}^{t \wedge T_{(i)}} \frac{A_{k}(s)}{1+A_{k}(s)} d A_{k}(s) \\
& =\sum_{i=1}^{n} \int_{T_{(i-1)}}^{t \wedge T_{(i)}} \frac{A_{(i) k}(s)}{1+A_{(i) k}(s)} d A_{(i) k}(s) .
\end{aligned}
$$

Notemos que o efeito de reparo mínimo do componente $k$, no compensador da $i$ falha se dá através do $i$-ésimo termo da última soma. Como $B_{(i)}(t)=\sum_{j=1}^{n} B_{(i) j}$ temos

$$
\begin{aligned}
& =1\left(T_{(i-1)}<t \leq T_{(i)}\right) \cdot\left[\sum_{j \neq k} A_{(i) j}+\int_{T_{(i-1)}}^{t \wedge T_{(i)}} \frac{A_{(i) k}(s)}{1+A_{(i) k}(s)} d A_{(i) k}(s)\right] \\
& =1\left(T_{(i-1)}<t \leq T_{(i)}\right) \cdot\left[\sum_{j \neq k} A_{(i) j}+\int_{T_{(i-1)}}^{t \wedge T_{(i)}} \frac{A_{k}(s)}{1+A_{k}(s)} d A_{k}(s)\right] .
\end{aligned}
$$

Desde que $E\left[A_{k}(T k)\right]=1$, pelo teorema de Girsanov definimos uma medida de probabilidade $Q_{\mu_{k}}$, com

$$
\frac{d Q_{\mu_{k}}}{d P}=A_{k}\left(T_{k}\right)
$$

de forma que, sob tal medida, a transformação $B_{(i)}(t)$ é o $\mathfrak{F}_{t}$-compensador do processo $N_{(i)}(t)=$ $1\left(T_{(i)} \leq t\right)$ e o efeito de reparo minimo da transformação no componente $k$ na $i$-ésima falha é:

$$
B_{(i) k}(t)=\int_{0}^{t} \frac{A_{(i) k}(s)}{1+A_{(i) k}(s)} d A_{(i) k}(s)
$$

concluindo a demonstração. 
Realizando reparos mínimos em todos os componentes podemos definir uma nova medida de probabilidade $Q_{\mu}$ definida pela derivada de Radon-Nikodym

$$
\frac{d Q_{\mu}}{d P}=\prod_{k=1}^{n} A_{k}\left(T_{k}\right)
$$

Usando o teorema de Girsanov temos o seguinte corolário:

Corolário 3.2.1 Seja $A_{j}(t)$ o $\mathfrak{F}_{t}$-compensador de $N_{j}(t)=1\left(T_{j} \leq t\right)$ onde $T_{j}$ é um $\mathfrak{F}_{t}$-tempo de parada totalmente inacessivel, representando o tempo de vida do componente $j$. Considere a transformação

$$
B_{j}(t)=\int_{0}^{t} \frac{A_{j}(s)}{1+A_{j}(s)} d A_{j}(s)
$$

para $j, 1 \leq j \leq n$. Então o novo $\mathfrak{F}_{t}$-compensador da $i$-ésima falha, $A_{(i)}(t)$ é:

$$
B_{(i)}(t)=\sum_{j=1}^{n} B_{(i) j}
$$

onde

$$
B_{(i) k}(t)=\int_{0}^{t} \frac{A_{(i) k}(s)}{1+A_{(i) k}(s)} d A_{(i) k}(s)
$$

para todo $k, 1 \leq k \leq n$.

\subsubsection{Importância de Natvig}

Como em Bueno(2011) [7], definimos a importância da confiabilidade de Natvig para o componente $j$ como

$$
I_{j}^{N}=E_{Q_{j}}[T]-E_{P}[T]
$$

onde a medida $Q_{j}$ é dada pelo teorema de Radon-Nykodim

$$
\frac{d Q_{j}}{d P}=A_{j}\left(T_{j}\right)
$$

Analisaremos detalhamente $A_{(i) j}(t)$ e $Q_{j}(T>t)$.

Lema 3.2.2 Sejam $T_{1}, . ., T_{n} \mathfrak{F}_{t}$-tempos de parada totalmente inacessiveis representando os tempos de vida dos componentes de um sistema coerente $\Phi$ com tempo de vida $T$. Seja $A_{(i) j}$ o $\mathfrak{F}_{t^{-}}$ compensador de $N_{(i) j}(t)=1\left(T_{(i) j} \leq t\right)$ e $A_{j}$ o $\mathfrak{F}_{t}$-compensador de $N_{j}(t)=1\left(T_{j} \leq t\right)$ entãa:

a) $A_{(i) j}(t)$ é contínuo;

b) $A_{(i) j}\left(T_{(i) j}\right)$ tem distribuição exponencial de parâmetro 1; 
c) $A_{(i) j}\left(T_{(i) j}\right)=A_{j}\left(T_{j}\right) 1\left(X_{i}=j\right)$.

\section{Demonstração}

Item[a)]. Provaremos que $T_{(i) j}$ é um tempo de parada totalmente inacessível. Seja $S$ qualquer tempo de parada acessível, então

$$
\begin{aligned}
P\left(\omega: T_{(i) j}(\omega)=S(\omega)<\infty\right) & =P\left(\omega: T_{(i) j}(\omega)<\infty, S(\omega)<\infty, T_{(i) j}(\omega)=S(\omega)\right) \\
& =P\left(\omega: T_{j}(\omega)<\infty, X_{i}=j, T_{j}(\omega)=S(\omega)\right) \\
& \leq P\left(\omega: T_{j}(\omega)=S(\omega)<\infty\right)=0
\end{aligned}
$$

onde a última igualdade segue do fato que $T_{j}$ é totalmente inacessível. Segue, do teorema de decomposição de Doob-Meyer, o $\mathfrak{F}_{t}$-compensador de $1\left(T_{(i) j} \leq t\right), A_{(i) j}(t)$, é contínuo.

Item[b)] Consequência do teorema A.2.7

Item[c)] Pela definição de $\mathfrak{F}_{t}$-compensador, o processo $A_{j}(t) 1\left(X_{i}=j\right)$ é não-descrescente adaptado e previsível. Logo pela unicidade do $\mathfrak{F}_{t}$-compensador basta provar que $N_{(i) j}(t)-A_{j}(t) 1\left(X_{i}=j\right)$ é um $\mathfrak{F}_{t}$-martingal. Sabemos que $N_{j}(t)-A_{j}(t)$ é um $\mathfrak{F}_{t}$-martingal e como $\left\{X_{i}=j\right\} \in \mathfrak{F}_{0}$ temos que

$$
\left(N_{j}(t)-A_{j}(t)\right) 1\left(X_{i}=j\right)=N_{j}(t) 1\left(X_{i}=j\right)-A_{j}(t) 1\left(X_{i}=j\right)=N_{(i) j}(t)-A_{j}(t) 1\left(X_{i}=j\right)
$$

é um $\mathfrak{F}_{t}$-martingal, o que conclui a demonstração do lema.

Lema 3.2.3 Sejam $T_{1}, . ., T_{n} \mathfrak{F}_{t}$-tempos de parada independentes totalmente inacessiveis representando os tempos de vida dos componentes de um sistema coerente $\Phi$ com tempo de vida T. Seja $A_{(i) j}$, $o \mathfrak{F}_{t}$-compensador de $N_{(i) j}(t)=1\left(T_{(i) j} \leq t\right)$ e $F_{(i) j}(t)$ a distribuição $P\left(T_{(i) j} \leq t \mid \mathfrak{F}_{t}\right)$ padronizada. Suponha que os $A_{(i) j}$ são funções injetoras ${ }^{1}$ então:

a) Para todo $i, i \geq 1$ temos

$$
Q_{j}\left(T=T_{(i)}\right)=\sum_{k \neq j}^{n} P\left(T=T_{(i) k}\right)+E\left[\overline{F_{(i) j}}\left(Y_{(i) j}\right)\left(1-\ln \left(\overline{F_{(i) j}}\left(Y_{(i) j}\right)\right)\right)-\overline{F_{(i) j}}(T)\left(1-\ln \left(\overline{F_{(i) j}}(T)\right)\right)\right]
$$

b) A distribuição de $T_{(i)}$ com respeito a medida $Q_{j}$ é dada por

$$
Q_{j}\left(T_{(i)}>t\right)=\sum_{k \neq j}^{n} E\left[\overline{F_{(i) k}}(t)\right]+E\left[\left(1-\ln \left(\overline{F_{(i) j}}(t)\right)\right) \overline{F_{(i) j}}(t)\right]
$$

\section{Demonstração}

Item[a)]Calculando a probabilidade temos

$$
Q_{j}\left(T=T_{(i)}\right)=\sum_{k=1}^{n} Q_{j}\left(T=T_{(i) k}\right)
$$

\footnotetext{
${ }^{1}$ Por exemplo isso é certo quando o sistema é suportivo. Ver Apêndice Definição A.2.6, Arjas and Norros [2] e Norros [16].
} 


$$
\begin{aligned}
& =\sum_{k \neq j}^{n} E\left[A_{j}\left(T_{j}\right) 1\left(T=T_{(i) k}\right)\right]+E\left[A_{j}\left(T_{j}\right) 1\left(T=T_{(i) j}\right)\right] \\
& =\sum_{k \neq j}^{n} E\left[1\left(T=T_{(i) k}\right)\right]+E\left[A_{j}\left(T_{j}\right) 1\left(T=T_{(i) j}\right)\right] \\
& =\sum_{k \neq j}^{n} E\left[1\left(T=T_{(i) k}\right)\right]+E\left[A_{(i) j}\left(T_{(i) j}\right) 1\left(T=T_{(i) j}\right)\right]
\end{aligned}
$$

onde a terceira igualdade vem do fato que os $A_{j}\left(T_{j}\right)$ são independentes e identicamente distribuidas com distribuição exponencial de parâmetro 1, a quarta igualdade do lema 3.2 .2 c), logo

$$
\begin{aligned}
Q_{j}\left(T=T_{(i)}\right) & =\sum_{k \neq j}^{n} E\left[1\left(T=T_{(i) k}\right)\right]+E\left[A_{(i) j}\left(T_{(i) j}\right) 1\left(Y_{(i) j}<T_{(i) j} \leq T\right)\right] \\
& =\sum_{k \neq j}^{n} E\left[1\left(T=T_{(i) k}\right)\right]+E\left[A_{(i) j}\left(T_{(i) j}\right) 1\left(A_{(i) j}\left(Y_{(i) j}\right)<A_{(i) j}\left(T_{(i) j}\right) \leq A_{(i) j}(T)\right)\right]
\end{aligned}
$$

onde a última igualdade provem do fato que os $A_{(i) j}(t)$ são funções injetoras. Usando a Observação 6 do Apêndice A e o lema 3.2.2 a), $A_{(i) j}(t)=-\ln \overline{F_{(i) j}}(t)$, onde $F_{(i) j}(t)=P\left(T_{(i) j} \leq t \mid \mathfrak{F}_{t}\right)$ e portanto

$$
\begin{aligned}
Q_{j}\left(T=T_{(i)}\right) & =\sum_{k \neq j}^{n} E\left[1\left(T=T_{(i) k}\right)\right]+E\left[\int_{A_{(i) j}\left(Y_{(i) j}\right)}^{A_{(i) j}(T)} x e^{-x} d x\right] \\
& =\sum_{k \neq j}^{n} E\left[1\left(T=T_{(i) k}\right)\right]+E\left[\overline{F_{(i) j}}\left(Y_{(i) j}\right)\left(1-\ln \left(\overline{F_{(i) j}}\left(Y_{(i) j}\right)\right)\right)-\overline{F_{(i) j}}(T)\left(1-\ln \left(\overline{F_{(i) j}}(T)\right)\right)\right]
\end{aligned}
$$

Item[b)] Calcularemos a distribuição de $T_{(i)}$ com a medida de probabilidade $Q_{j}$

$$
\begin{aligned}
Q_{j}\left(T_{(i)}>t\right) & =\sum_{k=1}^{n} Q_{j}\left(T_{(i) k}>t\right) \\
& =\sum_{k=1}^{n} E\left[A_{j}\left(T_{j}\right) 1\left(T_{(i) k}>t\right)\right] \\
& =\sum_{k \neq j}^{n} E\left[1\left(T_{(i) k}>t\right)\right]+E\left[A_{j}\left(T_{j}\right) 1\left(T_{(i) j}>t\right)\right] \\
& =\sum_{k \neq j}^{n} E\left[1\left(T_{(i) k}>t\right)\right]+E\left[A_{(i) j}\left(T_{(i) j}\right) 1\left(T_{(i) j}>t\right)\right] \\
& =\sum_{k \neq j}^{n} E\left[1\left(T_{(i) k}>t\right)\right]+E\left[\int_{A_{(i) j}(t)}^{\infty} x e^{-x} d x\right]
\end{aligned}
$$


onde a segundaigualdade é devido ao fato que os $A_{j}\left(T_{j}\right)$ são independentes e identicamente distribuidas com distribuição exponencial de parametro 1 e, a quarta e quinta igualdade sai do lema 3.2.2 parte c) e a) respectivamente. Da fórmula de Dolean (Observação 6 do Apêndice A) e o lema 3.2.2 a), $A_{(i) j}(t)=-\ln \overline{F_{(i) j}}(t)$, onde $F_{(i) j}(t)=P\left(T_{(i) j} \leq t \mid \mathfrak{F}_{t}\right)$. Assim

$$
Q_{j}\left(T_{(i)}>t\right)=\sum_{k \neq j}^{n} E\left[\overline{F_{(i) k}}(t)+E\left[\left\{1-\ln \overline{F_{(i) j}}(t)\right\} \overline{F_{(i) j}}(t)\right]\right.
$$

terminando a demonstração do lema.

Teorema 3.2.1 Seja $T$ o tempo de vida de um sistema coerente de ordem n e $T_{1}, . ., T_{n}$ os $\mathfrak{F}_{t}$-tempos de parada totalmente inacessiveis independentes e identicamente distribuidos com distribuição $F$, representando os tempos de vida dos componentes. Suponha que os $A_{(i) j}$ são funções injetoras. Então a Importância da confiabilidade de Natvig para o componente $j$ é:

$$
I_{j}^{N}=-\sum_{i=1}^{n} \alpha_{i j} E\left[\int_{0}^{\infty} \overline{F_{(i) j}}(t) \ln \overline{F_{(i) j}}(t) d t\right]
$$

onde $\alpha_{i j}=P\left(T=T_{(i) j}\right)=I_{(i) j}$

Demonstração Primeiro provaremos que sob a medida $Q_{j}$ as variáveis $T_{1}, . ., T_{n}$ são independentes.

$$
\begin{aligned}
Q_{j}\left(T_{1} \leq t_{1}, . ., T_{n} \leq t_{n}\right) & =E\left[A_{j}\left(T_{j}\right) 1\left(T_{j} \leq t_{j}\right)\right] \prod_{k \neq j}^{n} E\left[1\left(T_{k} \leq t_{k}\right)\right] \\
& =E\left[A_{j}\left(T_{j}\right) 1\left(T_{j} \leq t_{j}\right)\right] \prod_{k \neq j}^{n} E\left[A_{j}\left(T_{j}\right) 1\left(T_{k} \leq t_{k}\right)\right] \\
& =E_{Q_{j}}\left[1\left(T_{j} \leq t_{j}\right)\right] \prod_{k \neq j}^{n} E_{Q_{j}}\left[1\left(T_{k} \leq t_{k}\right)\right] \\
& =\prod_{k=1}^{n} Q_{j}\left[T_{k} \leq t_{k}\right]
\end{aligned}
$$

onde a segunda igualdade vale porque $A_{j}\left(T_{j}\right)$ é independente dos $T_{k}$, para $k \neq j$ e $E\left[A_{j}\left(T_{j}\right)\right]=1$.

Como as variáveis $T_{1}, . ., T_{n}$ são independentes, usando a decomposição por assinaturas das probabilidades $P(T>t)$ e $Q_{j}(T>t)$ temos

$$
\begin{aligned}
P(T>t) & =\sum_{i=1}^{n} \sum_{k=1}^{n} P\left(T=T_{(i) k}\right) P\left(T_{(i) k}>t\right) \\
& =\sum_{i=1}^{n} \sum_{k \neq j}^{n} \alpha_{(i) k} P\left(T_{(i) k}>t\right)+\sum_{i=1}^{n} \alpha_{(i) j} E\left[1\left(A_{(i) j}\left(T_{(i) j}\right)>A_{(i) j}(t)\right)\right] \\
& =\sum_{i=1}^{n} \sum_{k \neq j}^{n} \alpha_{(i) k} P\left(T_{(i) k}>t\right)+\sum_{i=1}^{n} \alpha_{(i) j} E\left[\int_{A_{(i) j}(t)}^{\infty} e^{-x} d x\right]
\end{aligned}
$$


onde na última igualdade usamos que os $A_{(i) j}\left(T_{(i) j}\right)$ têm distribuição exponencial de parâmetro 1.

$$
\begin{aligned}
Q_{j}(T>t) & =\sum_{i=1}^{n} \sum_{k=1}^{n} Q_{j}\left(T=T_{(i) k}, T_{(i) k}>t\right) \\
& =\sum_{i=1}^{n} \sum_{k=1}^{n} E\left[1\left(T=T_{(i) k}\right) 1\left(T_{(i) k}>t\right) A_{j}\left(T_{j}\right)\right] \\
& =\sum_{i=1}^{n} \sum_{k \neq j}^{n} \alpha_{(i) k} E\left[1\left(T_{(i) k}>t\right) A_{j}\left(T_{j}\right)\right]+\sum_{i=1}^{n} \alpha_{(i) j} E\left[1\left(T_{(i) j}>t\right) A_{j}\left(T_{j}\right)\right] \\
& =\sum_{i=1}^{n} \sum_{k \neq j}^{n} \alpha_{(i) k} P\left(T_{(i) k}>t\right)+\sum_{i=1}^{n} \alpha_{(i) j} E\left[1\left(A_{(i) j}\left(T_{(i) j}\right)>A_{(i) j}(t)\right) A_{j}\left(T_{j}\right)\right] \\
& =\sum_{i=1}^{n} \sum_{k \neq j}^{n} \alpha_{(i) k} P\left(T_{(i) k}>t\right)+\sum_{i=1}^{n} E\left[\int_{A_{(i) j}(t)}^{\infty} x e^{-x} d x\right]
\end{aligned}
$$

onde na quarta linha vem da hipótese que os $A_{(i) j}$ são injetivos e na última igualdade usamos que os $A_{(i) j}$ tem distribuicao exponencial de parâmetro 1 . Portanto temos que

$$
\begin{aligned}
I_{j}^{N} & =\int_{0}^{\infty}\left(Q_{j}(T>t)-P(T>t)\right) d t \\
& =\int_{0}^{\infty} \sum_{i=1}^{n} \alpha_{i j} E\left[\int_{A_{(i) j}(t)}^{\infty}(x-1) e^{-x} d x\right] d t \\
& =-\sum_{i=1}^{n} \alpha_{i j} E\left[\int_{0}^{\infty} \overline{F_{(i) j}}(t) \ln \overline{F_{(i) j}}(t) d t\right]
\end{aligned}
$$

onde a última igualdade segue do fato que $A_{(i) j}=-\ln \overline{F_{(i) j}}(t)$, terminando assim a demonstração do teorema.

É interessante perguntar como a assinatura é afetada por reparos mínimos em todos os componentes, analisando a decomposição através de assinatura sob a nova probabilidade $Q_{\mu}$ definida por o teorema de Radon-Nykodim:

$$
\frac{d Q_{\mu}}{d P}=\prod_{j=1}^{n} \frac{d Q_{j}}{d P}=\prod_{j=1}^{n} A_{j}\left(T_{j}\right) .
$$

A resposta é dada pelo seguinte teorema.

Teorema 3.2.2 Seja $T$ o tempo de vida de um sistema coerente de ordem n e $T_{1}, . ., T_{n}$ os $\mathfrak{F}_{t}$-tempos de parada totalmente inacessiveis independentes e identicamente distribuidos com distribuição $F$, representando os tempos de vida dos componentes. Suponha que o $A_{(i) j}$ compensador de $N_{(i) j}$ é uma função injetora, para todo $i, j$. Então, depois de um reparo minimo em todos os componentes 
do sistema temos que no intervalo $\left\{T_{(i)} \leq t<T_{(i+1)}\right\}$ :

$$
Q_{\mu}\left(T>t \mid \mathfrak{F}_{t}^{*}\right)=\sum_{k=i+1}^{n} \frac{Q_{\mu}\left(T=T_{(k)}\right)}{Q_{\mu}\left(T \geq T_{(i+1)}\right)} 1\left(T_{(k)}>t\right)
$$

onde

a) Para $k \geq i+1$ temos

$$
Q_{\mu}\left(T=T_{(k)}\right)=\sum_{i=1}^{n} E\left[\overline{F_{(k) i}}\left(Y_{(k) i}\right)\left(1-\ln \left(\overline{F_{(k) i}}\left(Y_{(k) i}\right)\right)\right)-\overline{F_{(k) i}}(T)\left(1-\ln \left(\overline{F_{(k) i}}(T)\right)\right)\right]
$$

b) $Q_{\mu}\left(T \geq T_{(i+1)}\right)$ é dado por $\sum_{k=i+1}^{n} Q_{\mu}\left(T=T_{(k)}\right)$;

c) A distribuição de $T_{(i)}$ com respeito a medida $Q_{\mu}$ é dada por

$$
Q_{\mu}\left(T_{(i)}>t\right)=\sum_{j=1}^{n} E\left[\left(1-\ln \left(\overline{F_{(i) j}}(t)\right)\right) \overline{F_{(i) j}}(t)\right] .
$$

onde $F_{(i) j}(t)=P\left(T_{(i) j} \leq t \mid \mathfrak{F}_{t}\right)$.

\section{Demonstração}

Primeiro provaremos que na medida de probabilidade $Q_{\mu}$, os $\mathfrak{F}_{t}$-tempos de vida $T_{1}, . ., T_{n}$ são independentes. Para isso calcularemos a distribuição conjunta dos $\mathfrak{F}_{t}$-tempos de vida $T_{1}, . ., T_{n}$ com respeito a $Q_{\mu}$.

$$
Q_{\mu}\left(T_{1} \leq t_{1}, . ., T_{n} \leq t_{n}\right)=E_{Q_{\mu}}\left[\prod_{k=1}^{n} 1\left(T_{k} \leq t_{k}\right)\right]=E\left[\prod_{k=1}^{n} A_{k}\left(T_{k}\right) 1\left(T_{k} \leq t_{k}\right)\right] .
$$

Pela independência das variáveis $A_{k}\left(T_{k}\right)$ e dos $\mathfrak{F}_{t}$-tempos de vida $T_{1}, . ., T_{n}$ temos que

$$
\begin{aligned}
Q_{\mu}\left(T_{1} \leq t_{1}, . ., T_{n} \leq t_{n}\right) & =E\left[\prod_{k=1}^{n} A_{k}\left(T_{k}\right) 1\left(T_{k} \leq t_{k}\right)\right] \\
& =\prod_{k=1}^{n} E\left[A_{k}\left(T_{k}\right) 1\left(T_{k} \leq t_{k}\right)\right] \\
& =\prod_{k=1}^{n} E_{Q_{\mu_{k}}}\left[1\left(T_{k} \leq t_{k}\right)\right] \\
& =\prod_{k=1}^{n} Q_{\mu}\left[T_{k} \leq t_{k}\right]
\end{aligned}
$$

e portanto os $\mathfrak{F}_{t}$-tempos de vida $T_{1}, . ., T_{n}$ são independentes com respeito a probabilidade $Q_{\mu}$. 
Para provar que são identicamente distribuidos sob a medida de probabilidade $Q_{\mu}$ basta notar que como os $\mathfrak{F}_{t}$-tempos de vida $T_{1}, . ., T_{n}$ são identicamente distribuidos, os $\mathfrak{F}_{t}$-compensadores dos $1\left(T_{j} \leq t\right)$ são idênticos e portanto os $T_{1}, . ., T_{n}$ são identicamente distribuidos sob $Q_{\mu}$.

Item[a)]Calculando diretamente

$$
\begin{aligned}
Q_{\mu}\left(T=T_{(i)}\right) & =\sum_{j=1}^{n} Q_{\mu}\left(T=T_{(i) j}\right) \\
& =\sum_{j=1}^{n} E\left[\prod_{k=1}^{n} A_{k}\left(T_{k}\right) 1\left(T=T_{(i) j}\right)\right] \\
& =\sum_{j=1}^{n} E\left[A_{j}\left(T_{j}\right) 1\left(T=T_{(i) j}\right)\right] \\
& =\sum_{j=1}^{n} E\left[A_{(i) j}\left(T_{(i) j}\right) 1\left(T=T_{(i) j}\right)\right]
\end{aligned}
$$

onde a terceira igualdade vem do fato que os $A_{j}\left(T_{j}\right)$ são independentes e identicamente distribuidos com distribuição exponencial de parâmetro 1, a quarta igualdade do lema 3.2.2 c), portanto

$$
\begin{aligned}
Q_{\mu}\left(T=T_{(i)}\right) & =\sum_{j=1}^{n} E\left[A_{(i) j}\left(T_{(i) j}\right) 1\left(Y_{(i) j}<T_{(i) j} \leq T\right)\right] \\
& =\sum_{j=1}^{n} E\left[A_{(i) j}\left(T_{(i) j}\right) 1\left(A_{(i) j}\left(Y_{(i) j}\right)<A_{(i) j}\left(T_{(i) j}\right) \leq A_{(i) j}(T)\right)\right]
\end{aligned}
$$

onde a última igualdade sai do fato que os $A_{(i) j}(t)$ são funções injetoras. Usando $A_{(i) j}=-\ln \overline{F_{(i) j}}(t)$, onde $F_{(i) j}(t)=P\left(T_{(i) j} \leq t \mid \mathfrak{F}_{t}\right)$ temos

$$
\begin{aligned}
Q_{\mu}\left(T=T_{(i)}\right) & =\sum_{j=1}^{n} E\left[\int_{A_{(i) j}\left(Y_{(i) j}\right)}^{A_{(i) j}(T)} x e^{-x} d x\right] \\
& =\sum_{j=1}^{n} E\left[\overline{F_{(k) i}}\left(Y_{(k) i}\right)\left(1-\ln \left(\overline{F_{(k) i}}\left(Y_{(k) i}\right)\right)\right)-\overline{F_{(k) i}}(T)\left(1-\ln \left(\overline{F_{(k) i}}(T)\right)\right)\right] .
\end{aligned}
$$

Item[b)]. É consequência direita do fato que $\left\{T \geq T_{(i)}\right\}$ pode ser escrita como união disjunta dos eventos $\left\{T=T_{(k)}\right\}$, para $k \geq i$.

Item[c)] Calcularemos a distribuição de $T_{(i)}$ com a medida de probabilidade $Q_{\mu}$

$$
\begin{aligned}
Q_{\mu}\left(T_{(i)}>t\right) & =\sum_{j=1}^{n} Q_{\mu}\left(T_{(i) j}>t\right) \\
& =\sum_{j=1}^{n} E\left[\prod_{k=1}^{n} A_{k}\left(T_{k}\right) 1\left(T_{(i) j}>t\right)\right]
\end{aligned}
$$




$$
\begin{aligned}
& =\sum_{j=1}^{n} E\left[A_{j}\left(T_{j}\right) 1\left(T_{(i) j}>t\right)\right] \\
& =\sum_{j=1}^{n} E\left[A_{(i) j}\left(T_{(i) j}\right) 1\left(T_{(i) j}>t\right)\right] \\
& =\sum_{j=1}^{n} E\left[\int_{A_{(i) j}(t)}^{\infty} x e^{-x} d x\right]
\end{aligned}
$$

onde a terceira igualdade sai do fato que os $A_{j}\left(T_{j}\right)$ são independentes e identicamente distribuidos com distribuição exponencial de parametro $1 \mathrm{e}$, a quarta e quinta igualdade vem do lema 3.2.2 parte c) e a), respectivamente. Da fórmula de Dolean $A_{(i) j}=-\ln \overline{F_{(i) j}}(t)$, onde $F_{(i) j}(t)=P\left(T_{(i) j} \leq t \mid \mathfrak{F}_{t}\right)$. Assim

$$
Q_{\mu}\left(T_{(i)}>t\right)=\sum_{j=1}^{n} E\left[\left\{1-\ln \overline{F_{(i) j}}(t)\right\} \overline{F_{(i) j}}(t)\right]
$$

terminando a demonstração de todos os items.

\subsection{Teoria de Valores Extremos e Assinaturas}

Em teoria da confiabilidade temos grande interesse na dinâmica do sistema no tempo, em particular em estimar a probabilidade de que um sistema usado, com idade $u$, falhe em um tempo adicional de $x$ unidades de tempo, $P(T-u \leq x \mid T>u)$. Por exemplo, se o sistema é um componente eletrónico que tem funcionado $u$ unidades de tempo, estamos interessados na probabilidade que o componente eletrônico falhe no intervalo $[u, u+x]$.

Nesta seção analisaremos tal questão no contexto das assinaturas, em que a estrutura do sistema é uma função das estatísticas de ordem e os resultados analíticos decorrem da teoria de valores extremos. Em um primeiro lugar enunciaremos resultados da teoria de valores extremos, já conhecidos, que serão usado para demonstrar o teorema principal.

Sejam $X_{1}, . ., X_{n}$ variáveis aleátorias independentes e identicamente distribuidas com distribuição $F$. A distribuição do máximo das $n$ observações, $M_{n}=\max \left\{X_{1}, . ., X_{n}\right\}$ é dada por

$$
P\left(M_{n} \leq x\right)=P\left(X_{1} \leq x, . ., X_{n} \leq x\right)=F^{n}(x)
$$

porque as variáveis são independentes e identicamente distribuidas. Suponhamos que seja possível encontrar sequências de números reais $a_{n}>0, b_{n} \in \mathbb{R}$, e uma função de distribuição $H(x)$ não degenerada tal que $\left(M_{n}-b_{n}\right) / a_{n}$ converge em distribuição:

$$
\begin{aligned}
P\left(\left(M_{n}-b_{n}\right) / a_{n} \leq x\right) & =P\left(M_{n} \leq a_{n} x+b_{n}\right) \\
& =F^{n}\left(a_{n} x+b_{n}\right) \rightarrow H(x)
\end{aligned}
$$


quando $n$ tende ao infinito. Quando essa condição é satisfeita, dizemos que $F$ pertence ao dominio maximo de atração de $H$, denotado por $F \in M D A(H)$. O seguinte teorema carateriza as possíveis funções de distribuição límite.

Teorema 3.3.1 Se existe constantes $a_{n}>0, b_{n} \in \mathbb{R}$ e uma distribuição não-degenerada $H$ tal que

$$
\frac{M_{n}-b_{n}}{a_{n}} \stackrel{D}{\rightarrow} H
$$

então $H$ tem uma das seguintes formas:

$$
\begin{gathered}
\Phi_{\delta}=\left\{\begin{array}{cc}
\exp \left\{-x^{-\delta}\right\}, & x>0 \\
0, & x \leq 0
\end{array}\right. \\
\Psi_{\delta}=\left\{\begin{array}{cc}
1, & x>0 \\
\exp \left\{-(-x)^{\delta}\right\}, & x \leq 0
\end{array}\right. \\
\Lambda(x)=\exp (-\exp (-x))
\end{gathered}
$$

Onde $\Psi_{\delta}$ é a distribuição de Frechét, $\Psi_{\delta}$ é a distribuição de Weibull e $\Lambda$ é a distribuição de Gumbel. Demonstração Ver Fisher-Tippett(1928) [12], Gnedenko(1943) [13].

A caracterização de cada distribuição esta dado pelo seguinte teorema.

Teorema 3.3.2 Seja F uma função de distribuição então:

a) $F \in M D A\left(\Phi_{\delta}\right)$ se, e somente se $x_{F}=\sup \{x: F(x)<1\}$ é infinito $e$

$$
\lim _{x \rightarrow \infty} \frac{1-F(\lambda x)}{1-F(x)}=\lambda^{\delta}
$$

para todo $\lambda$ positivo.

b) $F \in M D A\left(\Psi_{\delta}\right)$ se, e somente se $x_{F}=\sup \{x: F(x)<1\}$ é finito e

$$
\lim _{x \rightarrow \infty} \frac{1-F\left(x_{F}-\frac{1}{\lambda x}\right)}{1-F\left(x_{F}-\frac{1}{x}\right)}=\lambda^{-\delta}
$$

para todo $\lambda$ positivo.

c) $F \in M D A(\Lambda)$ se, e somente se

$$
\lim _{x \rightarrow x_{F}} \frac{1-F(x+t b(x))}{1-F(x)}=\exp (-t)
$$


para todo $t$ positivo, e para alguma função positiva $b($.$) . Se a igualdade for valida para alguma$ função b(.) então $\int_{x}^{x_{F}}(1-F(s)) d s<\infty$ para $x<x_{F}$ e podemos identificar

$$
b(x)=\frac{\int_{x}^{x_{F}}(1-F(s)) d s}{1-F(x)}
$$

Demostração. Ver DeHaan [10]

Lema 3.3.1 Seja F uma função de distribuição absolutamente contínua com função de densidade $f$, e $F_{(k)}$ a função de distribuição da k-ésima estatística de ordem de n-variáveis independentes e identicamente distribuidas com função de distribuição F. Então

a) Se $F \in M D A\left(\Phi_{\delta}\right)$ temos que $F_{(k)} \in M D A\left(\Phi_{(n-k+1) \delta}\right)$;

b) $S e F \in M D A\left(\Psi_{\delta}\right)$ temos que $F_{(k)} \in M D A\left(\Psi_{(n-k+1) \delta}\right)$;

c) $S e F \in M D A(\Lambda)$ temos que $F_{(k)} \in M D A(\Lambda)$.

\section{Demonstração}

item[a)]. Do teorema anterior, $F \in M D A\left(\Phi_{\delta}\right)$ se, e somente se $x_{F}=\sup \{x: F(x)<1\}$ é infinito e

$$
\lim _{x \rightarrow \infty} \frac{1-F(\lambda x)}{1-F(x)}=\lambda^{\delta}
$$

Analisaremos o comportamento de $F_{(k)}$. Da continuidade de $F$, temos que $x_{F_{(k)}}=x_{F}$. Calculemos o límite

$$
\begin{aligned}
\lim _{x \rightarrow \infty} \frac{1-F_{(k)}(\lambda x)}{1-F_{(k)}(x)} & =\lim _{x \rightarrow \infty} \frac{\int_{\lambda x}^{1} F^{k-1}(x)(1-F(x))^{n-k} d F(x)}{\int_{x}^{1} F^{k-1}(x)(1-F(x))^{n-k} d F(x)} \\
& =\lim _{x \rightarrow \infty}\left[\frac{F(\lambda x)}{F(x)}\right]^{k-1}\left[\frac{1-F(\lambda x)}{1-F(x)}\right]^{n-k}\left[\frac{f(\lambda x) \lambda}{f(x)}\right] \\
& =\lambda^{(n-k) \delta} \cdot \lambda^{\delta}=\lambda^{(n-k+1) \delta}
\end{aligned}
$$

onde a segunda linha segue da regra de l'Hospital, e a terceira do fato que $F \in M D A\left(\Phi_{\delta}\right)$. Assim temos provado que $F_{(k)} \in M D A\left(\Phi_{(n-k+1) \delta}\right)$.

item[b)] Para provar que $F_{(k)} \in M D A\left(\Psi_{\delta}\right)$, basta provar, pelo teorema anterior, que $x_{F_{(k)}}=\sup \{x$ : $\left.F_{(k)}(x)<1\right\}$ é finito e

$$
\lim _{x \rightarrow \infty} \frac{1-F_{(k)}\left(x_{F_{(k)}}-\frac{1}{\lambda x}\right)}{1-F_{(k)}\left(x_{F_{(k)}}-\frac{1}{x}\right)}=\lambda^{-\delta}
$$

Analisaremos o comportamento de $F_{(k)}$. Da continuidade de $F$, temos que $x_{F_{(k)}}=x_{F}$ e

$\lim _{x \rightarrow \infty} \frac{1-F_{(k)}\left(x_{F}-\frac{1}{\lambda x}\right)}{1-F_{(k)}\left(x_{F}-\frac{1}{x}\right)}=\lim _{x \rightarrow \infty} \frac{\int_{x_{F}-\frac{1}{\lambda x}}^{1} F^{k-1}(x)(1-F(x))^{n-k} d F(x)}{\int_{x_{F}-\frac{1}{x}}^{1} F^{k-1}(x)(1-F(x))^{n-k} d F(x)}$ 


$$
\begin{aligned}
& =\lim _{x \rightarrow \infty}\left[\frac{F\left(x_{F}-\frac{1}{\lambda x}\right)}{F\left(x_{F}-\frac{1}{x}\right)}\right]^{k-1}\left[\frac{1-F\left(\lambda x_{F}-\frac{1}{\lambda x}\right)}{1-F\left(x_{F}-\frac{1}{x}\right)}\right]^{n-k}\left[\frac{f\left(x_{F}-\frac{1}{\lambda x}\right)}{f\left(x_{F}-\frac{1}{x}\right)} \frac{x^{2} \lambda}{x^{2}}\right] \\
& =\lambda^{-(n-k) \delta} \cdot \lambda^{-\delta}=\lambda^{-(n-k+1) \delta}
\end{aligned}
$$

onde a segunda linha é consequência da regra de l'Hospital, e a terceira é proveniente do fato que $F$ pertence ao dominio de atração de $\Psi_{\delta}$. Assim temos provado que $F_{(k)} \in M D A\left(\Psi_{(n-k+1) \delta}\right)$.

item $[\mathrm{c})]$ Do teorema anterior, $F \in M D A(\Lambda)$ se, e somente se

$$
\lim _{x \rightarrow x_{F}} \frac{1-F(x+t b(x))}{1-F(x)}=\exp (-t)
$$

para alguma função $b(x)$ positiva. Do fato que $F \in M D A(\Lambda)$, podemos escolher $b(x)$ da forma

$$
b(x)=\frac{\int_{x}^{x_{F}}(1-F(s)) d s}{1-F(x)}
$$

Para provar que $F_{(k)} \in M D A(\Lambda)$, escolhamos $b k(x)=b(x) /(n-k+1)$. Assim, usando a regra de l'Hospital e o fato que $F \in M D A(\Lambda)$

$$
\begin{aligned}
& \lim _{x \rightarrow x_{F}} \frac{1-F_{(k)}(x+t b k(x))}{1-F_{(k)}(x)}=\lim _{x \rightarrow x_{F}} \frac{\int_{x+t b k(x)}^{1} F^{k-1}(x)(1-F(x))^{n-k} d F(x)}{\int_{x}^{1} F^{k-1}(x)(1-F(x))^{n-k} d F(x)} \\
= & \lim _{x \rightarrow x_{F}}\left[\frac{F(x+t b k(x))}{F(x)}\right]^{k-1}\left[\frac{1-F(x+t b k(x))}{1-F(x)}\right]^{n-k}\left[\frac{f(x+t b k(x)) t b k^{\prime}(x)}{f(x)}\right] \\
= & \lim _{x \rightarrow x_{F}}\left[\frac{F(x+t b k(x))}{F(x)}\right]^{k-1}\left[\frac{1-F(x+t b(x) /(n-k+1))}{1-F(x)}\right]^{n-k}\left[\frac{f\left(x+t \frac{b(x)}{(n-k+1)}\right) t \frac{b^{\prime}(x)}{(n-k+1)}}{f(x)}\right] \\
= & \left(\exp \left(-\frac{t}{n-k+1}\right)\right)^{n-k} \cdot \exp \left(-\frac{t}{n-k+1}\right)=\exp \left(-\frac{t}{n-k+1}\right)(n-k+1)=\exp (-t)
\end{aligned}
$$

o que implica que $F_{(k)} \in M D A(\Lambda)$, terminando a demonstração do teorema.

Definição 3.3.1 (Distribuição de Valores Extremos Generalizada) Definimos a seguinte função de distribuição $H_{\gamma}$ por

$$
H_{\gamma}=\left\{\begin{array}{cc}
\exp \left\{-(1+\gamma x)^{-1 / \gamma}\right\}, & \gamma \neq 0 \\
\exp \{-\exp (-x)\}, & \gamma=0
\end{array}\right.
$$

onde $1+\gamma x>0$. O suporte é

$$
x>-\frac{1}{\gamma} \text { se } \gamma>0
$$




$$
\begin{array}{rrr}
x<-\frac{1}{\gamma} & \text { se } & \gamma<0 \\
x \in \mathbb{R} & \text { se } & \gamma=0
\end{array}
$$

A função de distribuição $H_{\gamma}$ é chamada distribuição de valores extremos generalizada (GEV), $e$ unifica as distribuições $\Phi_{\delta}, \Phi_{\delta}$ e $\Lambda$ em uma familia paramétrica com parâmetro $\gamma=1 / \delta$.

O lema anterior pode ser lido da seguinte forma: Se $F \in M D A\left(H_{\gamma}\right)$ então $F_{(k)} \in M D A\left(H_{\gamma /(n-k+1)}\right)$.

Definição 3.3.2 (Distribuição de Pareto Generalizada)

Definimos a seguinte função de distribuição $G_{\gamma}$ por

$$
G_{\gamma}(x)=\left\{\begin{array}{cc}
1-(1+\gamma x)^{-1 / \gamma}, & \gamma \neq 0 \\
1-\exp (-x), & \gamma=0
\end{array}\right.
$$

com $x \in D(\gamma)$, onde

$$
D(\gamma)=\left\{\begin{array}{cc}
0 \leq x<\infty, & \gamma \geq 0 \\
0 \leq x \leq-1 / \gamma, & \gamma<0
\end{array}\right.
$$

A função de distribuição $G_{\gamma}$ é chamada distribuição de Pareto generalizada (GPD).

Teorema 3.3.3 (Teorema de Pickands-Balkema-de Haan)

Para $\gamma \in \mathbb{R}$ são equivalentes:

a) $F \in M D A\left(H_{\gamma}\right)$

b) Existe uma função mensurável estritamente positiva $\beta($.$) tal que$

$$
\lim _{u \uparrow x_{F}} \operatorname{Sup}_{x \in\left(0, x_{F}-u\right)}\left|F_{u}(x)-G_{\gamma, 0, \beta(u)}(x)\right|=0
$$

onde $F_{u}=P(X-u \leq x \mid X>u)$ é denominada função de distribuição de excesso da distribuição $F$ acima do valor u e $G_{\gamma, 0, \beta(u)}$ denota a familia de distribuições $G_{\gamma, \mu, \beta}$ relacionada a $G P D$ definida por

$$
G_{\gamma, \mu, \beta}(x)=1-\left(1+\gamma \frac{x-\mu}{\beta}\right)^{-1 / \gamma}
$$

Demonstração. Ver Pickands [17], Balkema e de Haan [3], e Degen [11].

Estamos interessado em estimar a probabilidade de que o sistema coerente usado, com idade $u$, falhe antes de um tempo adicional de $x$ unidades de tempo, $F_{u}=P(T-u \leq x \mid T>u)$, no contexto das assinaturas. Usando a teoria de valores extremos temos o seguinte teorema. 
Teorema 3.3.4 Sejam $T_{1}, . ., T_{n} \mathfrak{F}_{t}$-tempos de parada independentes e identicamente distribuidos com distribuição $F$, representando os tempos de vida dos componentes de um sistema coerente $\Phi$ com tempo de vida T. Se F é uma função de distribuição absolutamente contínua com função de densidade $f$ e $F \in M D A\left(H_{\gamma}\right)$. Então:

A função de distribuição de excesso do tempo de vida $T, F_{u}=P(T-u \leq x \mid T>u)$ converge uniformente, quando u tende a $x_{T}$, à distribução de Pareto generalizada,

$$
G_{\frac{\gamma}{n-k+1}, 0, \beta_{k}(u)}
$$

onde $k$ é o maior número com $\alpha_{k}=P\left(T=T_{(k)}\right)$ diferente de zero, e $\beta_{k}(u)$ é uma função mensurável estritamente positiva.

Demonstração Como os $T_{1}, . ., T_{n}$ são $\mathfrak{F}_{t}$-tempos de parada independentes e identicamente distribuidos com distribuição $F$, representando os tempos de vida dos componentes de um sistema coerente $\Phi$ com tempo de vida $T$, da decomposição por assinaturas temos

$$
\begin{aligned}
F_{u}(x) & =P(T-u \leq x \mid T>u)=\frac{P(T \leq u+x, T>u)}{P(T>u)} \\
& =\frac{P(T \leq u+x)-P(T \leq u)}{P(T>u)} \\
& =\sum_{i=1}^{n} \alpha_{i} P\left(T_{(i)} \leq x+u \mid T_{(i)}>u\right) \frac{P\left(T_{(i)}>u\right)}{P(T>u)}
\end{aligned}
$$

Analisaremos os termos de cada parcela $P\left(T_{(i)} \leq x+u \mid T_{(i)}>u\right)$ e $P\left(T_{(i)}>u\right) / P(T>u)$ quando $u$ tende a $x_{T}$. Primeiro $P\left(T_{(i)} \leq x+u \mid T_{(i)}>u\right)$. Como $F \in M D A\left(H_{\gamma}\right)$, do lema 3.3.1, segue que $T_{(i)} \in M D A\left(H_{\gamma /(n-i+1)}\right)$ e do teorema de Pickands-Balkema-de Haan temos que existe uma função mensurável $\beta_{i}($.$) tal que { }^{2}$ :

$$
\lim _{u \uparrow x_{T}} \operatorname{Sup}_{x \in\left(0, x_{T}-u\right)}\left|P\left(T_{(i)} \leq x+u \mid T_{(i)}>u\right)-G_{\gamma / n-i+1,0, \beta_{i}(u)}(x)\right|=0 .
$$

Passemos a analisar cada $P\left(T_{(i)}>u\right) / P(T>u)$. Seja $k$ é o maior número com $\alpha_{k}$ diferente de zero. Como $F$ é uma função de distribuição absolutamente contínua com função de densidade $f$ temos usando a regra de l'Hôpital que:

$$
\lim _{u \uparrow x_{T}} \frac{P\left(T_{(i)}>u\right)}{P\left(T_{(k)}>u\right)}= \begin{cases}0, & i<k \\ 1, & i=k .\end{cases}
$$

Assim

$$
\lim _{u \uparrow x_{T}} \frac{P\left(T_{(i)}>u\right)}{\sum_{j=1}^{k} \alpha_{j} P\left(T_{(j)}>u\right)}=\left\{\begin{array}{cc}
0, & i<k \\
1 / \alpha_{k}, & i=k .
\end{array}\right.
$$

\footnotetext{
${ }^{2}$ Podemos escrever $x_{T}$ em vez de $x_{T_{(i)}}$ devido à decomposição de assinaturas, $P(T>t)=\sum_{i=1}^{n} \alpha_{i} P\left(T_{(i)}>t\right)$
} 
Denotemos o limite de $P\left(T_{(i)}>u\right) / P(T>u)$, quando $u$ tende a $x_{T}$, por $q_{i}$.

Se denotamos por $M(u, i)=\operatorname{Sup}_{x \in\left(0, x_{F}-u\right)}\left|P\left(T_{(i)} \leq x+u \mid T_{(i)}>u\right)-G_{\gamma / n-i+1,0, \beta_{i}(u)}(x)\right|$ temos que

$$
\begin{aligned}
\mid P\left(T_{(i)}\right. & \left.\leq x+u \mid T_{(i)}>u\right) \frac{P\left(T_{(i)}>u\right)}{P(T>u)}-q_{i} G_{\gamma / n-i+1,0, \beta_{i}(u)}(x) \mid \\
& \leq G_{\gamma / n-i+1,0, \beta_{i}(u)}(x)\left|\frac{P\left(T_{(i)}>u\right)}{P(T>u)}-q_{i}\right|+M(u, i) \frac{1}{\alpha_{i}} \\
& \leq\left|\frac{P\left(T_{(i)}>u\right)}{P(T>u)}-q_{i}\right|+M(u, i) \frac{1}{\alpha_{i}}
\end{aligned}
$$

converge uniformemente a zero, quando $u$ tende a $x_{T}$. A última linha sai do fato que os GPD são funções de distribuições. Portanto, se denotamos por $\lambda_{i}$ o produto $\alpha_{i} q_{i}$, temos

$$
\begin{gathered}
\left|P(T \leq x+u \mid T>u)-\sum_{i=1}^{n} \lambda_{i} G_{\gamma / n-i+1,0, \beta_{i}(u)}(x)\right| \\
\leq\left|\sum_{i=1}^{n} \alpha_{i} P\left(T_{(i)} \leq x+u \mid T_{(i)}>u\right) \frac{P\left(T_{(i)}>u\right)}{P(T>u)}-\sum_{i=1}^{n} \alpha_{i} q_{i} G_{\gamma / n-i+1,0, \beta_{i}(u)}(x)\right| \\
\leq \sum_{i=1}^{n} \alpha_{i}\left|P\left(T_{(i)} \leq x+u \mid T_{(i)}>u\right) \frac{P\left(T_{(i)}>u\right)}{P(T>u)}-q_{i} G_{\gamma / n-i+1,0, \beta_{i}(u)}(x)\right|
\end{gathered}
$$

converge uniformente a zero, quando $u$ tende a $x_{F}$. Usando o fato que $\lambda_{k}=1$ e $\lambda_{i}$ é zero para o resto de índices temos que

$$
\left|P(T \leq x+u \mid T>u)-G_{\gamma / n-k+1,0, \beta_{k}(u)}(x)\right|=\left|P(T \leq x+u \mid T>u)-\sum_{i=1}^{n} \lambda_{i} G_{\gamma / n-i+1,0, \beta_{i}(u)}(x)\right|
$$

converge converge uniformente a zero, quando $u$ tende a $x_{F}$, terminando a demonstração do teorema.

Como consequência temos o seguente corolario.

Corolário 3.3.1 Sejam $T_{1}, . ., T_{n} \mathfrak{F}_{t}$-tempos de parada independentes e identicamente distribuidos com distribuição $F$, representando os tempos de vida dos componentes de um sistema coerente $\Phi$ com tempo de vida T. Sejam $T^{1}, . ., T^{m}$ tempos de vida independentes e identicamente distribuidos, com a mesma função de distribuição que o tempo de vida $T$ do sistema $\Phi$. Se F é uma função de distribuição absolutamente contínua com função de densidade $f$ e $F \in M D A\left(H_{\gamma}\right)$. Então, o tempo de vida do sistema em paralelo com componentes $T^{j}$ converge, convenientemente padronizada, em distribuição à função de não degenerada $H_{\gamma / n-k+1}, k$ é o maior número com $\alpha_{k}=P\left(T=T_{(k)}\right)$ diferente de zero e $\alpha$ é o vetor de assinatura do sistema $\Phi$. 


\section{Demonstração}

Do teorema anterior temos que

$$
\lim _{u \uparrow x_{T}} \operatorname{Sup}_{x \in\left(0, x_{T}-u\right)}\left|P(T \leq x+u \mid T>u)-G_{\gamma / n-k+1,0, \beta_{k}(u)}(x)\right|=0 .
$$

Usando o teorema de Pickands-Balkema-de Haan, concluimos que $T \in M D A\left(H_{\gamma / n-k+1}\right)$, é dizer, existem sequências de números $a_{m} \in \mathbb{R}^{+}, b_{m} \in \mathbb{R}$ tais que

$$
\left(\max \left\{T^{1}, . ., T^{m}\right\}-b_{m}\right) / a_{m} \stackrel{D}{\rightarrow} H_{\gamma / n-k+1}
$$

quando $m$ tende ao infinito, onde as variáveis aleatórias $\left\{T^{k}: k \in \mathbb{N}\right\}$ são independentes e identicamente distribuidas com a mesma distribuição que o tempo de vida $T$ do sistema $\Phi$. 


\section{Capítulo 4}

\section{Conclusões}

\subsection{Considerações Finais}

Neste trabalho, estendemos a noção de assinaturas para o caso de dependência dos tempos de vida dos componentes. Provamos que sob diferentes níveis de informação, o tempo de vida de um sistema coerente se expressa, ainda, como combinação linear das estatísticas de ordem dos tempos de vida dos componentes. Este ponto de vista é de caracter prático, porque quando analisamos um sistema fisicamente, a informação a disposição é dada pela falhas dos componentes ordenados, isto é, sabemos qual componente falhou e o instante exato de sua falha. Analisamos tambem as diferentes medidas de importâncias da confiabilidade dos componentes para a confiabilidade do sistema e o tempo de vida residual de um sistema coerente no contexto das assinaturas.

\subsection{Sugestões para Pesquisas Futuras}

- Analisar classes de distribuições úteis na teoria da confiabilidade, no contexto das assinaturas, como $M I F R\left|\mathfrak{F}_{t}, N B U\right| \mathfrak{F}_{t}$, etc;

- Estudar desigualdades estocásticas entre sistema coerentes baseada nas desigualdades estocásticas entre suas assinaturas;

- Estudar relações assintóticas do tempo de vida do sistema coerente no contexto das assinaturas;

- Procurar relações entre a importância dos componentes e as assinaturas dinâmicas. 


\section{Apêndice A}

\section{Apêndice}

Nesta seção revisaremos alguns resulatdos da teoria dos processos pontuais. Por simplicidade de notação vamos assumir que as relações entre conjuntos mensuráveis e variables aleatórias, como $\subset, \subseteq, \leq,<,=$ serão sempre com probabilidade um, assim expressões ou termos como P-q.c geralmente serão omitido. Para terminar, com respeito as esperanças condicionais não faremos distinção entre uma versão e a classe de equivalência P-q.c dessa versão.

\section{A.1 Processos Estocásticos}

Seja $(\Omega, \mathfrak{F}, P)$ um espaço de probabilidade e $(E, \mathcal{E})$ um espaço topológico. Um processo estocástico com valores em $E$ é uma função $X: \mathbb{R}^{+} \times \Omega \rightarrow E$ mensurável com respeito à $\sigma$-álgebra produto $\mathcal{B}\left(\mathbb{R}^{+}\right) \times \mathfrak{F}$, onde $\mathcal{B}\left(\mathbb{R}^{+}\right)$é a $\sigma$-álgebra gerada pelos borelianos em $\mathbb{R}^{+}$.

Com a definição de processo estocástico associamos a definição de filtragem.

Definição A.1.1 O conjunto $\left(\mathfrak{F}_{t}, t \geq 0\right)$ é uma filtragem em $(\Omega, \mathfrak{F})$ se para cada $t \geq 0$, $\mathfrak{F}_{t}$ é uma sub- $\sigma$-álgebra de $\mathfrak{F}$ e para todo $s \leq t$

$$
\mathfrak{F}_{s} \subset \mathfrak{F}_{t}
$$

Se, em adição, para todo $t \geq 0$ temos que

$$
\mathfrak{F}_{t}=\bigcap_{h>0} \mathfrak{F}_{t+h}
$$

dizemos que a filtragem é contínua à direita.

Se $\left(\mathfrak{F}_{t}, t \geq 0\right)$ é contínua à direita e completa, dizemos que $\left(\mathfrak{F}_{t}, t \geq 0\right)$ satisfaz as condições de Dellacherie.

Denotamos por $\mathfrak{F}_{\infty}$ a menor $\sigma$-álgebra que contém todos os $\mathfrak{F}_{t}, \mathfrak{F}_{\infty}=\sigma\left(\cup_{t \geq 0} \mathfrak{F}_{t}\right)$.

Definição A.1.2 O processo estocástico $\left(X_{t}, t \geq 0\right)$ é $\mathfrak{F}_{t}$-progressivo se, para todo $t \geq 0$, a função $X_{s}(w)=X(s, w)$ para $(s, w) \in[0, t] \times \Omega$ é $\mathcal{B}[0, t] \times \mathfrak{F}_{t}$-mensurável. Se $X_{t}$ é $\mathfrak{F}_{t}$-mensurável dizemos que o processo é adaptado a $\mathfrak{F}_{t}$.

Teorema A.1.1 Seja $(E, \mathcal{E})$ um espaço topológico metrizável e seja $X_{t}$ um processo estocástico adaptado a $\mathfrak{F}_{t}$. Se $X_{t}$ é contínuo à direita (ou contínuo à esquerda) então $X_{t}$ é $\mathfrak{F}_{t}$-progressível. 
Demostração Bremaud [6].

Além das noções de mensurabilidade e progressividade uma noção importante é a noção de previsibilidade.

Definição A.1.3 Seja $\mathfrak{F}_{t}$ uma filtragem associada ao espaço de probabilidade $(\Omega, \mathfrak{F}, P)$. Definimos a $\sigma$-álgebra $\mathfrak{F}_{t}$-previsivel sobre $(0, \infty) \times \Omega$ e denotamos por $\mathcal{P}\left(\mathfrak{F}_{\mathfrak{t}}\right)$ ou simplesmente $\mathcal{P}_{t}$ à $\sigma$-álgebra sobre $(0, \infty) \times \Omega$ gerada por elementos da forma

$$
(s, t] \times A ; A \in \mathfrak{F}_{s} ; s \leq t .
$$

Um processo estocástico com valores em $\mathbb{R}$ é $\mathfrak{F}_{t}$-previsível se a função $(t, w) \rightarrow X_{t}(w)$ definida em $(0, \infty) \times \Omega$ e $\mathcal{P}\left(\mathfrak{F}_{\mathfrak{t}}\right)$-mensurável.

O teorema seguinte fornece um método fácil de verificar se um processo é $\mathfrak{F}_{t}$-previsível

Teorema A.1.2 Qualquer processo $X_{t}$ com valores em $\mathbb{R}$, adaptado a $\mathfrak{F}_{t}$ continuo à esquerda é um processo $\mathfrak{F}_{t}$-previsível.

Demostração Bremaud [6].

Observe que, na hierarquia de mensurabilidade, a noção de previsibilidade é a mais forte:

Teorema A.1.3 Se o processo $X_{t}$ é $\mathfrak{F}_{t}$-previsivel então $X_{t}$ é $\mathfrak{F}_{t}$-progressivel.

Demostração Bremaud [6].

\section{A.1.1 Tempo de Parada}

Seja $\left(\mathfrak{F}_{t}, t \geq 0\right)$ uma filtragem de $(\Omega, \mathfrak{F})$. Uma variável aleátoria $T:(\Omega, \mathfrak{F}) \rightarrow[0, \infty]$ é chamada Tempo de Parada com respeito a $\left(\mathfrak{F}_{t}, t \geq 0\right)$ ou simplesmente $\mathfrak{F}_{t}$-tempo de parada, se para todo $t \geq 0$

$$
\{T \leq t\} \in \mathfrak{F}_{t}
$$

Associado ao tempo de parada $T$ temos duas $\sigma$-álgebras, relacionadas ao passado de $T$ e ao passado estrito de $T$.

Definição A.1.4 Seja $T$ um $\mathfrak{F}_{t}$-tempo de parada. Então o passado no tempo de parada T, é dado pela $\sigma$-álgebra $\mathfrak{F}_{T}$ definida como

$$
\mathfrak{F}_{T}=\left\{A \in \mathfrak{F}_{\infty}: A \cap\{T \leq t\} \in \mathfrak{F}_{t}, t \geq 0\right\}
$$

$O$ passado estrito em $T$ é a $\sigma$-álgebra $\mathfrak{F}_{T-\text { definida como }}$

$$
\mathfrak{F}_{T-}=\sigma\left\{A_{0} \in \mathfrak{F}_{0}, A_{s} \cap\{T>s\}: A_{s} \in \mathfrak{F}_{s}, s \geq 0\right\}
$$


Algumas definições que relacionam os conceitos de tempo de parada e de previsibilidade são:

Definição A.1.5 Seja $T$ um $\mathfrak{F}_{t}$-tempo de parada então:

a) T e um $\mathfrak{F}_{t^{-}}$tempo de parada previsível se existe uma sequência crescente $\left(T_{n}, n \geq 0\right)$ de $\mathfrak{F}_{t^{-}}$ tempos de paradas com $T_{n}<T$ e lim $T_{n}=T$. Tal sequência é dita que anuncia $T$.

b) $T$ e um $\mathfrak{F}_{t}$-tempo de parada totalmente inacessivel se $P(T=\tau<\infty)=0$ para todo $\tau$, $\mathfrak{F}_{t}$-tempo de parada previsivel.

\section{A.1.2 Martingal}

Definição A.1.6 (Martingal, Submartingal e Supermartingal) Seja $\left(\mathfrak{F}_{t}, t \geq 0\right)$ uma filtragem em $(\Omega, \mathfrak{F})$. Um processo $M_{t}$ com valores reais é chamado $\left(P, \mathfrak{F}_{t}\right)$-martingal sobre $[0, d]$ (onde d é um numero real não negativo) se

a) $M_{t}$ é adaptado a $\mathfrak{F}_{t}$;

b) $M_{t}$ é $\mathfrak{F}_{t}$-integrável, $E\left[\left|M_{t}\right|\right]<\infty, t \in[0, d]$;

c) $E\left[M_{t} \mid \mathfrak{F}_{s}\right]=M_{s}$ P-q.c, para todo $0 \leq s \leq t$.

Se $M_{t}$ é um $\left(P, \mathfrak{F}_{t}\right)$-martingal sobre $[0, d]$ para todo $d \geq 0$ então $M_{t}$ é chamado $\left(P, \mathfrak{F}_{t}\right)$-martingal. Se no item $[c)]$ a igualdade é substituida por $E\left[M_{t} \mid \mathfrak{F}_{s}\right] \leq M_{s}, M_{t}$ é chamado $\left(P, \mathfrak{F}_{t}\right)$-supermartingal. Se no item $[c)]$ a igualdade é substituida por $E\left[M_{t} \mid \mathfrak{F}_{s}\right] \geq M_{s}, M_{t}$ é chamado $\left(P, \mathfrak{F}_{t}\right)$-submartingal.

Alguns teoremas importantes na teoria dos martingais são:

Teorema A.1.4 (Teorema de Amostragem Opcional)

Seja $M_{t}$ um $\mathfrak{F}_{t}$-supermartingal contínuo à direita com $S$ e $T$ dois tempos de parada finitos tais que $S \leq T$. Então :

a) Se T é limitada por uma constante ( e portanto S tambem é limitada) ou

b) Se $M_{t}$ é uniformente integrável, isto é

$$
\lim _{A \uparrow \infty} \operatorname{Sup}\left\{\int_{\left|M_{t}\right|>A}\left|M_{t}\right| d P: t \geq 0\right\}=0
$$

então

$$
E\left[M_{T} \mid \mathfrak{F}_{S}\right] \leq M_{S}
$$

Observação 5 Quando $M_{t}$ é um $\mathfrak{F}_{t}$-martingal continuo à direita temos que

$$
E\left[M_{T} \mid \mathfrak{F}_{S}\right]=M_{S}
$$


como consequência direta do teorema de amostragem opcional desde que $M_{t} e-M_{t}$ são supermartingais.

Demostração Protter [18].

Teorema A.1.5 Seja $\left(M_{t}, t \geq 0\right)$ é um $\mathfrak{F}_{t}$-martingal que é uniformente integrável, então existe uma variável aleátoria $M_{\infty}$ tal que

$$
M_{\infty}=\lim _{t \rightarrow \infty} M_{t}
$$

$e$

$$
M_{t}=E\left[M_{\infty} \mid \mathfrak{F}_{t}\right]
$$

Demostração Protter [18].

Lema A.1.1 (Lema de Komatsu)

Seja $\left(X_{t}, t \geq 0\right)$ um processo $\mathcal{F}_{t}$-progressivo tal que para todo tempo de parada $T, X_{T}$ é integrável e $E\left[X_{T}\right]=E\left[X_{0}\right]$. Então o processo $\left(X_{t}, t \geq 0\right)$ é um $\mathcal{F}_{t}$-martingal.

Demostração Bremaud [6].

Teorema A.1.6 (Formula Integral de Dellacherie)

Seja $\left(A_{t}, t \geq 0\right)$ um processo $\mathcal{F}_{t}$-adaptado crescente e $\left(M_{t}, t \geq 0\right)$ um $\mathfrak{F}_{t}$-martingal não negativo, continuo à direita e uniformente integrável. Então para qualquer tempo de parada $T$, adaptado à filtragem $\mathfrak{F}_{t}$, temos

$$
E\left[\int_{0}^{T} M_{s} d A_{s}\right]=E\left[M_{T} A_{T}\right]
$$

Demostração Protter [18].

Por razões técnicas a noção de martingal não é suficiente e necessitamos de um conceito mais geral, o conceito de martingal local.

Definição A.1.7 Seja $X_{t}$ um processo $\mathfrak{F}_{t}$-adaptado e $\left(S_{n}, n \geq 1\right)$ uma familia de $\mathfrak{F}_{t}$-tempos de parada tais que:

a) $\lim _{n \rightarrow \infty} S_{n}=\infty$;

b) Para cada $n \geq 1, X_{t \wedge S_{n}}$ é um $\mathfrak{F}_{t}$-martingal.

Então $X_{t}$ é chamado $\left(P, \mathfrak{F}_{t}\right)$-martingal local, ou $\mathfrak{F}_{t}$-martingal local quando $P$ é evidente. 
Teorema A.1.7 (Decomposição de Doob-Meyer)

Seja $\left(X_{t}, t \geq 0\right)$ um processo $\mathcal{F}_{t}$-adaptado, contínuo à direita e com límite à esquerda. Então $X_{t}$ é um $\mathcal{F}_{t}$-submartingal uniformente integrável se, e somente se, o processo $\left(X_{t}, t \geq 0\right)$ possui uma decomposição da forma

$$
X_{t}=M_{t}+A_{t}
$$

onde $\left(A_{t}, t \geq 0\right)$ é um processo $\mathcal{F}_{t}$-previsivel, crescente, contínuo à direita, com $A_{0}=0$ e $\left(M_{t}, t \geq 0\right)$ é um $\mathcal{F}_{t}$-martingal uniformente integrável. Essa decomposição é única.

Demostração Protter [18].

Definição A.1.8 (Semimartingal Regular)

Seja $\left(X_{t}, t \geq 0\right)$ um processo estocástico $\mathcal{F}_{t}$-adaptado. Dizemos que $\left(X_{t}, t \geq 0\right)$ é um semimartingal regular se ele tem uma decomposição da forma:

$$
X_{t}=X_{0}+\int_{0}^{t} f_{s} d s+M_{t}
$$

onde $\left(f_{t}, t \geq 0\right)$ um processo estocástico progressivamente mensurável com a propriedade que para todo $t \geq 0$

$$
E\left[\int_{0}^{t}\left|f_{s}\right| d s\right]<\infty
$$

$E\left|X_{0}\right|$ é finito e $\left(M_{t}, t \geq 0\right)$ é um $\mathcal{F}_{t}$-martingal contínuo à direita com límite à esquerda e $M_{0}=0$.

Tal processo se diz que tem uma decomposição do tipo SMR.

\section{A.2 Processos Pontuais}

Primeiro, por simplicidade, estudaremos o que é um processo pontual univariado, ou simplesmente um processo pontual. Um processo pontual é uma sequência $\left(T_{n}, n \geq 1\right)$ crescente, de variáveis aleatórias positivas que podem assumir o valor infinito. A partir de agora assumiremos que o processo é não explosivo, isto é:

$$
T_{\infty}=\lim _{n \rightarrow \infty} T_{n}=\infty
$$

Como $\left(T_{n}, n \geq 1\right)$ é uma sequência crescente o limite existe.

Uma maneira equivalente de definir um processo pontual é definir o processo de contagem associado a $\left(T_{n}, n \geq 1\right)$ :

$$
N_{t}=\sum_{k=1}^{\infty} 1\left(T_{k} \leq t\right)
$$


que está bem definido se assumimos que o processo $\left(T_{n}, n \geq 1\right)$ é não explosivo. Para cada realização $\omega,\left(N_{t}(\omega), t \geq 0\right)$, é uma função escada, contínua à direita com limite à esquerda e com saltos de tamanho 1 . O processo $\left(N_{t}, t \geq 0\right)$ conta o número de pontos $T_{n}$ que ocorrem até o instante de tempo $t$.

Com a definição de processo pontual relacionamos o conceito de compensador.

Definição A.2.1 Seja $\left(N_{t}, t \geq 0\right)$ um processo de contagem integrável, adaptado a uma filtragem $\mathfrak{F}_{t}$, e seja $\Lambda_{t}$ um processo $\mathfrak{F}_{t}$-previsível, integrável e não-decrescente tal que a diferença

$$
M_{t}=N_{(t)}-\Lambda_{t}
$$

é um $\mathfrak{F}_{t}$-martingal. Então o processo $\Lambda_{t}$ é chamado o $\mathfrak{F}_{t}$-compensador do processo $\left(N_{t}, t \geq 0\right)$. A existência do compensador é dada pelo Teorema da decomposição de Doob-Meyer.

Uma maneira alternativa de verificar tal condição é provar que a igualdade

$$
E\left[\int_{0}^{\infty} C_{s} d N_{s}\right]=E\left[\int_{0}^{\infty} C_{s} d \Lambda_{s}\right]
$$

é satisfeita para todo processo $\mathfrak{F}_{t}$-previsível não negativo $C_{t}$.

Intuitivamente o compensador satisfaz

$$
\Lambda_{t}=\lim _{h \rightarrow 0} \frac{1}{h} \int_{0}^{t}\left(E\left[N_{s+h} \mid \mathfrak{F}_{s}\right]-N_{s}\right) d s, \quad d \Lambda_{t}=E\left[d N_{t} \mid \mathfrak{F}_{t}\right] .
$$

Em alguns casos a medida gerada pelo compensador é absolutamente contínua com respeito é medida de Lebesgue o que nos leva à seguinte definição.

Definição A.2.2 Seja $\left(N_{t}, t \geq 0\right)$ um processo de contagem integrável adaptado a uma filtragem $\mathfrak{F}_{t}$, e $\lambda_{t}$ um processo $\mathfrak{F}_{t}$-progressivo tal que :

$$
\int_{0}^{t} \lambda_{s} d s<\infty
$$

Se para todo processo $C_{t}, \mathfrak{F}_{t}$-previsivel e não negativo, temos

$$
E\left[\int_{0}^{\infty} C_{s} d N_{s}\right]=E\left[\int_{0}^{\infty} C_{s} \lambda_{s} d s\right]
$$

dizemos que o processo $N=\left(N_{t}, t \geq 0\right)$ admite a intensidade $\lambda_{t}$. Note que $\lambda_{t}$ depende da filtragem $\mathfrak{F}_{t}$ e da probabilidade $P$, assim dizemos que $\lambda_{t}$ é $\left(P, \mathfrak{F}_{t}\right)$-intensidade. 
Se requeremos que $\lambda_{t}$ seja $\mathfrak{F}_{t}$-previsível, é possível demostrar que a intensidade é única. Por outro lado se existe intensidade para um processo pontual sempre é possível encontrar uma versão, dessa intensidade, que seja previsível.

Em geral o cálculo de intensidade é um trabalho árduo. No teorema seguinte temos um método para calcular a intensidade baseado nas densidades dos tempos entre as chegadas, do processo pontual.

Teorema A.2.1 Seja $\left(N_{t}, t \geq 0\right)$ um processo de contagem integrável adaptado a uma filtragem $\mathfrak{F}_{t}$, onde $\mathfrak{F}_{t}$ e o $\sigma$-álgebra natural associada a $\left(N_{t}, t \geq 0\right), \mathfrak{F}_{t}=\sigma\left\{N_{s}: 0 \leq s \leq t\right\}$. Suponhamos que $G_{n}(w, B)$ é a distribução condicional regular dos tempos entre as chegadas $U_{n+1}=T_{n+1}-T_{n}$, $n \geq 0$, e $T_{0}=0$ dado o passado $\mathfrak{F}_{T_{n}}$, isto é:

$$
G_{n}(w, B)=P\left(U_{n+1} \in B \mid \mathfrak{F}_{T_{n}}\right)=P\left(T_{n+1}-T_{n} \in B \mid \mathfrak{F}_{T_{n}}\right)
$$

para todo boreliano $B$.

Então

$$
A_{t}=A_{T_{n}}+\int_{0}^{t-T_{n}} \frac{G_{n}(d s)}{G_{n}[s, \infty]}
$$

no intervalo $\left\{T_{n}<t \leq T_{n+1}\right\}$.

Note que o compensador $A_{t}$ é aditivo!.

Se a distribução condicional regular dos tempos entre as chegadas $G_{n}$ admite uma densidade $g_{n}$, $n \in\{0,1,2, .$.$\} então a intensidade \mathfrak{F}_{t}$-previsivel $\lambda$ é dada por:

$$
\lambda_{t}=\sum_{n=0}^{\infty} \frac{g_{n}\left(t-T_{n}\right)}{1-\int_{0}^{t-T_{n}} g_{n}(s) d s} 1\left(T_{n}<t \leq T_{n+1}\right)
$$

Demostração Bremaud [6].

No caso particular de processos pontuais temos os seguintes teoremas.

Teorema A.2.2 Seja $\left(N_{t}, t \geq 0\right)$ um processo pontual $\mathcal{F}_{t}$-adaptado com intensidade $\lambda_{t}$, e $M_{t}=$ $N_{t}-\int_{0}^{t} \lambda_{s} d s$ o martingal associado e seja $\left(X_{t}, t \geq 0\right)$ e um processo $\mathcal{F}_{t}$-previsivel $t \geq 0$

a) $\mathrm{Se}$

$$
E\left[\int_{0}^{t}\left|X_{s}\right| \lambda_{s} d s\right]<\infty
$$

então $Y_{t}=\int_{0}^{t} X_{s} d M_{s}$ é um $\mathfrak{F}_{t}$-martingal

b) $S e$

$$
\int_{0}^{t}\left|X_{s}\right| \lambda_{s} d s<\infty, t \geq 0
$$


então $Y_{t}=\int_{0}^{t} X_{s} d M_{s}$ é um $\mathfrak{F}_{t}$-martingal local

Teorema A.2.3 Seja $T$ um tempo de parada associado à $\sigma$-álgebra $\left(\mathfrak{F}_{t}, t \geq 0\right)$.

a) Se o processo $\left(N_{t}, t \geq 0\right)$, com $N_{t}=1(T \leq t)$, tem uma representação do tipo $S M R$

$$
N_{t}=\int_{0}^{t} 1(T \geq s) \lambda_{s} d s+M_{t}
$$

então $T$ é $\mathfrak{F}_{t}$-tempo de parada totalmente inacessivel e $M_{t}$ é um $\mathfrak{F}_{t}$-martingal quadrado integrável de média zero.

b) Se $T$ é $\mathfrak{F}_{t}$-tempo de parada totalmente inacessível então o processo associado $\left(N_{t}, t \geq 0\right)$ tem uma unica decomposição do tipo

$$
N_{t}=\Lambda_{t}+M_{t}
$$

onde $M_{t}$ é um $\mathfrak{F}_{t}$-martingal integrável e $\Lambda$ é contínuo.

\section{Demostração Protter [18].}

Uma generalização do processo pontual univariado é o conceito de processo pontual multivariado.

Definição A.2.3 Seja $\left(T_{n}, n \geq 1\right)$ um processo pontual univariado e $\left(Z_{n}, n \geq 1\right)$ uma sequência de variáveis aleatórias com valores em um alfabeto finito $\mathcal{A}=\left\{a_{1}, a_{2}, \ldots, a_{m}\right\}$. A sequência de pares $\left(T_{n}, Z_{n}\right)$ com $n \geq 1$ é chamado processo pontual multivariado. Com esta sequência de pares associamos um processo de contagem $N_{t}$ representando por um vetor de tamanho $m$, onde $m$ e o numero de elemento do alfabeto $\mathcal{A}$, da seguinte forma:

$$
N_{t}=\left(N_{t}(1), N_{t}(2), \ldots, N_{t}(m)\right)
$$

onde

$$
N_{t}(i)=\sum_{k \geq 1} 1\left(T_{k} \leq t\right) 1\left(V_{k}=a_{i}\right)
$$

para todo $i \in\{1,2 \ldots, m\}$.

\section{A.2.1 Fórmula Exponencial e Teorema de Girsanov}

Nesta subseção consideramos processos que são contínuos à direita com límite à esquerda. No desenvolvimento da dissertação, para estudar conceitos como importância de componentes, reparos mínimos utilizamos transformações nos compensadores. Um teorema importante para analisar tais transformações é o teorema de Girsanov. Para demonstra-lo usaremos os seguintes resultados:

Teorema A.2.4 (Fórmula do Produto)

Sejam $f$ e $g$ duas funções contínuas à direita com limites à esquerda e de variação limitada sobre qualquer intervalo finito. Então: 


$$
f(t) g(t)=f(0) g(0)+\int_{0}^{t} f(s) d g(s)+\int_{0}^{t} g(s-) d f(s)
$$

Observação: O produto de $f$ e $g, f(t) g(t)$, tambem pode ser escrito como

$$
f(0) g(0)+\int_{0}^{t} f(s-) d g(s)+\int_{0}^{t} g(s) d f(s)
$$

Teorema A.2.5 (Formula Exponêncial)

Sejam a uma função contínua à direita, crescente, com $a(0)=0$, e $\psi$ uma função tal que, para todo $t \geq 0$,

$$
\int_{0}^{t}|\psi(s)| d a(s)<\infty
$$

Então a equação

$$
x(t)=x(0)+\int_{0}^{t} x(s-) \psi(s) d a(s)
$$

admite uma única solução localmente limitada $\left(\sup _{s \in[0, t]}|x(s)|<\infty\right)$ dada pela formula

$$
x(t)=x(0) . \prod_{0<s \leq t}(1+\psi(s) \Delta a(s)) \cdot \exp \left(\int_{0}^{t} \psi(s) d a^{c}(s)\right)
$$

onde $\Delta a(t)=a(t)-a(t-)$ e $a^{c}(t)=a(t)-\sum_{s \leq t} \Delta a(s)$.

Demostração Ver Bremaud [6].

\section{Observação 6}

Como consequência existe uma correspondência bijetiva entre os espaço das funções de distribuição dos $\mathfrak{F}_{t}$-tempos de parada e o espaço dos $\mathfrak{F}_{t}$-compensadores

$$
P\left(T>t \mid \mathfrak{F}_{t}\right)=\exp \left[-A_{t}^{c}\right] \prod_{0<s \leq t}\left(1-\Delta A_{s}\right)
$$

onde $A_{t}$ é o $\mathfrak{F}_{t}$-compensador do processo $1(T \leq t), \Delta A_{t}=A_{t}-A_{t-}$ e $A_{s}^{c}=A_{t}-\sum_{0<s \leq t} \Delta A_{s}$ é a parte contínua de $A_{t}$.

O teorema de Girsanov nos permite realizar trasformãções no espaco dos $\mathfrak{F}_{t}$-compensadores.

Teorema A.2.6 (Teorema de Girsanov para Processos Pontuais)

Seja $\left(N_{t}(1), . ., N_{t}(k)\right)$ um processo pontual k-variado adaptado à filtragem $\mathfrak{F}_{t}$ e $\lambda_{t}(i), 1 \leq i \leq k a$ 
intensidade $\left(P, \mathfrak{F}_{t}\right)$-previsivel associada a $N_{t}(i)$. Seja $\mu_{t}(i), 1 \leq i \leq k$, um processo $\mathfrak{F}_{t}$-previsivel, não negativo tal que para $t \geq 0$ e $1 \leq i \leq k$

$$
\int_{0}^{t} \mu_{s}(i) \lambda_{s}(i) d s<\infty
$$

Seja $L_{t}$ o processo

$$
L_{t}=\prod_{i=1}^{k} L_{t}(i)
$$

onde o $L_{t}(i)$ é definido como

$$
L_{t}(i)=\prod_{n \geq 1} \mu_{T_{n}(i)}^{1\left(T_{n}(i) \leq t\right)} \cdot \exp \left(A_{t}(i)-B_{t}(i)\right)
$$

$\operatorname{com} A_{t}(i)=\int_{0}^{t} \lambda_{s}(i) d s$ e $B_{t}(i)=\int_{0}^{t} \mu_{s}(i) \lambda_{s}(i) d s=\int_{0}^{t} \mu_{s}(i) d A_{t}(i)$

Então $L_{t}$ é um $\left(P, \mathfrak{F}_{t}\right)$-martingal local não negativo e um $\left(P, \mathfrak{F}_{t}\right)$-supermartingal.

\section{Demostração}

Primeiro provaremos que o processo $L_{t}$ satisfaz a seguinte equação :

$$
L_{t}=1+\sum_{i=1}^{k} \int_{0}^{t} L_{s-\cdot} \cdot\left(\mu_{s}(i)-1\right) \cdot d M_{s}(i)
$$

onde $M_{s}(i)=N_{s}(i)-\int_{0}^{t} \lambda_{s}(i) d s$.

Da definição de $L_{t}(i)$ temos que se $\psi_{s}(i)=\mu_{s}(i)-1, M_{s}(i)=N_{s}(i)-\int_{0}^{t} \lambda_{s}(i) d s, \Delta M_{s}(i)=\Delta N_{s}(i)$ e $M_{t}(i)-\sum_{s \leq t} \Delta M_{s}(i)=M_{t}(i)-\sum_{s \leq t} \Delta N_{s}(i)=-\int_{0}^{t} \lambda_{s}(i) d s$ pois $N_{t}=\sum_{s \leq t} \Delta N_{s}(i)$. Assim temos que

$$
\begin{aligned}
\prod_{0<s \leq t}\left(1+\psi_{s}(i) \Delta N_{s}\right) & =\prod_{0<s \leq t}\left(1+\left(\mu_{s}(i)-1\right) \cdot \Delta N_{s}\right) \\
& =\prod_{n \geq 1} \mu_{T_{n}(i)}^{1\left(T_{n}(i) \leq t\right)}
\end{aligned}
$$

Usando a fórmula exponencial para o processo $L_{t}(i)$ temos:

$$
L_{t}(i)=1+\int_{0}^{t} L_{s-}(i) \cdot\left(\psi_{s}(i)\right) \cdot d M_{s}(i)
$$

onde $\psi_{s}(i)=\mu_{s}(i)-1$.

Usando a fórmula do produto repetida vezes para $L_{t}=\prod_{i=1}^{k} L_{t}(i)$ obtemos 


$$
L_{t}=\prod_{i=1}^{k} L_{t}(i)=1+\sum_{i=1}^{k} \int_{0}^{t} \prod_{m=1, m \neq i}^{k} L_{s-}(m) d L_{s}(i)
$$

$\operatorname{mas} d L_{s}(i)=L_{s-}(i) \cdot\left(\mu_{s}(i)-1\right) \cdot d M_{s}(i), 1 \leq i \leq k$. Portanto concluimos que

$$
L_{t}=1+\sum_{i=1}^{k} \int_{0}^{t} L_{s-} \cdot\left(\mu_{s}(i)-1\right) \cdot d M_{s}(i) .
$$

Usaremos tal expressão para provar que o processo $L_{t}$ é um martingal local e uma supermartingal.

Para provar que $L_{t}$ é um martingal local consideremos os tempos de parada

$$
S_{n}=\inf \left\{t>0: L_{t-} \geq n \text { ou } \sum_{i=1}^{k} \int_{0}^{t}\left|\mu_{s}(i)-1\right| d \lambda_{s}(i) \geq n\right\}
$$

se o conjunto $\left\{t: L_{t-} \geq n\right.$ ou $\left.\sum_{i=1}^{k} \int_{0}^{t}\left|\mu_{s}(i)-1\right| d \lambda_{s}(i) \geq n\right\}$ é não vazio e $S_{n}=\infty$ se for vazio. Logo o processo $L_{t \wedge S_{n}}$ é um martingal pois, para $t \leq S_{n}$, temos

$$
E\left[\sum_{i=1}^{k} \int_{0}^{t}\left|L_{s-.} \cdot\left(\mu_{s}(i)-1\right)\right| . d \lambda_{s}(i)\right]<\infty
$$

O que resta provar é que a sequência $\left(S_{n}, n \geq 0\right)$ tende ao infinito. Claramente a sequência é crescente, e se o límite fosse um numero real positivo finito teriamos uma contradicção com o fato que $\int_{0}^{t} \mu_{s}(i) \lambda_{s}(i) d s<\infty, \int_{0}^{t} \lambda_{s}(i) d s<\infty$ e $L_{t-}$ é um processo continuo à esquerda. Assim o processo $L_{t \wedge S_{n}}$ é um martingal e $L_{t}$ é um local martingal.

Para provar que $L_{t}$ é um $\mathfrak{F}_{t}$-supermartingal, usaremos o fato que $L_{t \wedge S_{n}}$ é um martingal

$$
E\left[L_{t \wedge S_{n}} \mid \mathfrak{F}_{s}\right]=L_{s \wedge S_{n}}
$$

e como os $L_{t}$ são positivos usamos o lema de Fatou para concluir que

$$
\begin{gathered}
E\left[L_{t} \mid \mathfrak{F}_{s}\right] \leq \lim _{n \rightarrow \infty} E\left[L_{t \wedge S_{n}} \mid \mathfrak{F}_{s}\right] \\
=\lim _{n \rightarrow \infty} L_{s \wedge S_{n}}=L_{s}
\end{gathered}
$$

isto é, $L_{t}$ é um supermartingal. 
Seja $\mathcal{G}$ uma $\sigma$-sub-álgebra de um espaço de probabilidade $(\Omega, \mathfrak{F}, P)$ e denotamos por $\mathcal{G} \cap A$ o traço da $\sigma$-álgebra $\mathcal{G}$ em $A$. Isto é

$$
\mathcal{G} \cap A=\{G \cap A / G \in \mathcal{G}\}
$$

onde $A$ é um subconjunto de $\Omega$. Claramente $\mathcal{G} \cap A$ é uma sub- $\sigma$-álgebra de $\mathcal{G}$ e se $A$ é um elemento de $\mathfrak{F}$ com probabilidade $P(A)$ positiva, definimos a probabilidade $P^{A}$ sobre $\mathcal{G} \cap A$ da seguinte maneira

$$
P^{A}(G \cap A)=\frac{P(G \cap A)}{P(A)}
$$

para $G \in \mathcal{G} . E^{A}[X \mid G \cap A]$ denota a esperança condicional da variável $X$ com respeito a probabilidade $P^{A}$ e a $\sigma$-álgebra $\mathcal{G} \cap A$.

Lema A.2.1 Seja $X$ uma variável integrável com respeito a $P$ e $\mathcal{G}$ uma sub- $\sigma$-álgebra. Então $X$ é $P^{A}$-integrável e se $A \in \mathcal{G}$ temos

$$
E^{A}[X \mid \mathcal{G} \cap A]=E[X \mid \mathcal{G}] \quad P^{A}-q . c .
$$

\section{Demonstração}

Seja $B \in \mathcal{G}$, então

$$
\begin{aligned}
\int 1(B) 1(A) E^{A}[X \mid \mathcal{G} \cap A] d P^{A} & =\int 1(B) 1(A) X d P^{A} \\
& =\frac{1}{P(A)} \int 1(B) 1(A) X d P \\
& =\frac{1}{P(A)} \int 1(B) E[1(A) X \mid \mathcal{G}] d P \\
& =\frac{1}{P(A)} \int 1(B) 1(A) E[1(A) X \mid \mathcal{G}] d P \\
& =\int 1(B) 1(A) E[1(A) X \mid \mathcal{G}] d P^{A}
\end{aligned}
$$

onde a segunda igualdade vem de $P^{A}(d x)=1(A) P(d x) / P(A)$ e a quarta igualdade sai do fato de que $A \in \mathcal{G}$. Temos, portanto, demostrado que

$$
\int 1(B) 1(A) E^{A}[X \mid \mathcal{G} \cap A] d P^{A}=\int 1(B) 1(A) E[1(A) X \mid \mathcal{G}] d P^{A}
$$

e como $E[1(A) X \mid \mathcal{G}]=1(A) E[X \mid \mathcal{G}]$ é $\mathcal{G} \cap A$-mensurável, da unicidade de esperança condicional, concluimos que $E^{A}[X \mid \mathcal{G} \cap A]=E[X \mid \mathcal{G}]$ quase-certamente com respeito a $P^{A}$.

Lema A.2.2 Seja $\left(N_{t}, t \geq 0\right)$ um processo de contagem integrável adaptado a uma filtragem $\mathfrak{F}_{t}, e$ 
$\Lambda_{t}$ seu $\mathfrak{F}_{t}$-compensador. Então

$$
E\left[\int_{0}^{\infty} 1(t<s) d N_{s} \mid \mathfrak{F}_{t}\right]=E\left[\int_{0}^{\infty} 1(t<s) d \Lambda_{s} \mid \mathfrak{F}_{t}\right]
$$

\section{Demonstração}

Seja $B \in \mathfrak{F}_{t}$ e consideremos o processo $C_{s}=1(t<s) 1(B)$. Este processo é $\mathfrak{F}_{s}$-previsível porque é contínua à esquerda e $\mathfrak{F}_{s}$-adaptada (devido a que $B \in \mathfrak{F}_{t} \subset \mathfrak{F}_{s}$ para todo $t<s$ ). Assim para todo $B \in \mathfrak{F}_{t}$ temos

$$
\begin{aligned}
\int 1(B) E\left[\int_{0}^{\infty} 1(t<s) d N_{s} \mid \mathfrak{F}_{t}\right] d P & =\int 1(B) \int_{0}^{\infty} 1(t<s) d N_{s} d P \\
& =\iint_{0}^{\infty} C_{s} d N_{s} d P \\
& =\iint_{0}^{\infty} C_{s} d \Lambda_{s} d P \\
& =\int 1(B) \int_{0}^{\infty} 1(t<s) d \Lambda_{s} d P \\
& =\int 1(B) E\left[\int_{0}^{\infty} 1(t<s) d \Lambda_{s}\right] d P
\end{aligned}
$$

onde a terceira igualdade vem da definição de $\mathfrak{F}_{t}$-compensador. $\square$

Teorema A.2.7 Sejam $T_{1}, \ldots, T_{n} \mathfrak{F}_{t}$-tempos de parada e os $A_{j}(t) \mathfrak{F}_{t}$-compensadores associados aos processos $N_{j}(t)=1\left(T_{j} \leq t\right)$ tais que:

a) Para todo $i, j$ com $i \neq j, P\left(T_{i}=T_{j}\right)=0$;

b) Todos os $\mathfrak{F}_{t}$-compensadores, $A_{j}(t)$, são continuos.

Então as variáveis aleátorias $A_{j}\left(T_{j}\right), 1 \leq j \leq n$ são independentes e identicamente distribuidas com distribuição exponencial de parâmetro 1.

A demonstração é baseada no artigo de Norros [16].

\section{Demonstração}

Para provar tal resultado é suficiente demonstrar que para qualquer $n$-vetor de números reais $\left(u_{1}, . ., u_{n}\right)$

$$
E\left[\exp \left(\sum_{j=1}^{n} i u_{j} A_{j}\left(T_{j}\right)\right)\right]=\prod_{j=1}^{n} \frac{1}{1-i u_{j}}
$$

onde $i$ é o numero complexo tal que $i^{2}=-1$.

Definimos os seguintes processos, para $1 \leq j \leq n$ 


$$
M_{t}(j)=\left(1-i u_{j}\right)^{N_{j}(t)} \cdot \exp \left\{i u_{j} A_{j}(t)\right\} .
$$

Da fórmula exponencial temos que os processos $M_{t}(j)$ satisfazem

$$
\begin{aligned}
& M_{t}(j)=1+\int_{0}^{t} M_{s-}(j) i u_{j} d\left(A_{j}(s)-N_{j}(s)\right) \\
& =1+\int_{0}^{t}\left(1-i u_{j}\right)^{N_{j}(s-)} i u_{j} d\left(A_{j}(s)-N_{j}(s)\right) .
\end{aligned}
$$

Portanto, os processos $M_{t}(j), 1 \leq j \leq n$ são martingais porque $A_{j}(t)-N_{j}(t)$ é um martingal, e $\left(1-i u_{j}\right)^{N_{j}(s-)}$ é $\mathfrak{F}_{t}$-previssível para cada $j$. Da continuidade dos processos $A_{j}(t), 1 \leq j \leq n$ e da hipótese que os processos $N_{j}(t), 1 \leq j \leq n$, não possuem saltos simultâneos temos que o produto dos martingais $\prod_{j \geq 1} M_{t}(j)$ é um martingal.

Como $A_{j}(\infty)=A_{j}\left(T_{j}\right)$ temos que

$$
E\left[\prod_{j \geq 1} M_{\infty}(j)\right]=E\left[\prod_{j \geq 1}\left(1-i u_{j}\right)^{N_{j}(\infty)} \cdot \exp \left(i u_{j} A_{j}(\infty)\right)\right]=E\left[\prod_{j \geq 1}\left(1-i u_{j}\right) \cdot \exp \left(i u_{j} A_{j}\left(T_{j}\right)\right)\right]
$$

e da propriedade de martingal (usando Kunita-Watanabe e a variação quadratica)

$$
E\left[\prod_{j \geq 1} M_{0}(j)\right]=E\left[\prod_{j \geq 1}\left(1-i u_{j}\right)^{N_{j}(0)} \cdot \exp \left(i u_{j} A_{j}(0)\right)\right]=E[1]=1
$$

o que termina a demostração do teorema.

É interessante notar que o resultado do teorema não depende das distribuições dos tempos de vida e da história do processo, desde que não apresentem falhas simultâneas.

Desde que o $\mathfrak{F}_{t}$-compensador depende da historia interna do processo, escrevemos o $\mathfrak{F}_{t}$-compensador $A_{k}(t)$ como

$$
A_{k}(t)=\sum_{i} 1\left(T_{(i)}<t \leq T_{(i+1)}\right) a_{t}^{(i)}\left(k, T_{(1)}, X_{1} ; . . ; T_{(n)}, X_{n}\right)
$$

onde cada $a_{(.)}^{(i)}(k ; \ldots)$ é uma função determinística em suas variáveis ( Ver Bremaud [6]). Assim o compensador $A_{k}($.$) é determinística por partes, cada falha de um componentes produz uma nova$ função para escolher.

Usamos as seguentes definições: Um conjunto de historias é um conjunto finito $H$, que consisite em pares $(t, i)$, com $t \in \mathbb{R}^{+}, i \in \mathcal{C}$ tal que se os pares $(s, i) \in H,(t, j) \in H$ e $s \neq t$ então $i \neq j$. Denotemos por $H^{+}$ao conjunto de componentes que estão em $H, H^{+}=\{i:(s, i) \in H\}$. Escrevemos $a_{t}\left[i ;\left\{\left(T_{(1)}, X_{1}\right), . .,\left(T_{(n)}, X_{n}\right)\right\}\right]$ em lugar de $a_{t}^{(i)}\left(t, k, T_{(1)}, X_{1} ; . . ; T_{(n)}, X_{n}\right)$. Definimos a historia do processo

$$
H_{t}=\left\{\left(T_{(k)}, X_{k}\right): T_{(k)}<t\right\}
$$


e assim escrevemos $A_{i}(t)=a_{t}\left(i ; H_{t}\right)$.

Suponhamos que o conjunto de funções $a_{t}(i ; H)$ satisfaz a condição A:

$$
a_{t}[i ; H \cup(s, j)]=a_{t}(i ; H), t \leq s .
$$

Definição A.2.4 Seja $H$, um conjunto de historias. Suponha que a familia de funções contínuas $\grave{a}$ direita, $a_{t}(i ; H)$, com $1 \leq i \leq n$, satisfaz a condição $A$. Então $a_{t}(i ; H)$ é chamada familia de compensadores do vetor $\left(T_{1}, . ., T_{n}\right)$, se cada processo $a_{t}\left(i ; H_{t}\right)$ é uma versão do compensador $A_{i}(t)$, com $1 \leq i \leq n$.

Com a familia de compensadores $\left\{a_{t}(i ; H)\right\}$ fixa, definimos

$$
S_{i}=a_{T_{i}}\left(i ; H_{T_{i}}\right)=A_{i}\left(T_{i}\right)
$$

para $i \in\{1, . ., n\}$. Denotamos por $\psi: \mathbb{R}_{+}^{n} \rightarrow \overline{\mathbb{R}}_{+}^{n}$ :

$$
\psi\left(T_{1}, . ., T_{n}\right)=\left(S_{1}, . ., S_{n}\right) .
$$

A função $\psi$ é chamada função de risco total do vetor $\left(T_{1}, . ., T_{n}\right)$. Esta função é 'bijetiva'. É possível expressar sua 'inversa' $\psi^{*}$ em termos da familia de compensadores do vetor $\left(T_{1}, . ., T_{n}\right)$ (Ver No$\operatorname{rros}[16])$.

Definição A.2.5 Sejam $H_{1}$ e $H_{2}$ dois conjuntos de historias. Dizemos que $H_{1}$ é menor que $H_{2}$, $H_{1} \leq H_{2}$, se $(t, i) \in H_{2}$ implica que $(s, i) \in H_{1}$, para algum $s \leq t$.

$H_{1} \leq H_{2}$ significa que as falhas em $H_{1}$ ocorrem antes e são mais numerosas que as falhas em $H_{2}$.

Definição A.2.6 Seja $\left\{a_{t}(i ; H)\right\}$ a familia de compensadores do vetor $\left(T_{1}, . ., T_{n}\right)$. Dizemos que a familia $\left\{a_{t}(i ; H)\right\}$ é suportiva se a seguente condição é satisfeita: Se $H_{1} \leq H_{2}$ e $i \notin H_{1}^{+}$então $a_{t}\left(i ; H_{1}\right) \geq a_{t}\left(i ; H_{2}\right)$.

O termo suportivo indica que os componentes suportam uns aos outros, no sentido que mais falhas e as mais recentes falhas aumentam o risco para o resto dos componentes que as poucas e posteriores falhas.

Proposição A.2.1 Se o vetor $\left(T_{1}, . ., T_{n}\right)$ tem uma familia de compensadores suportiva, então a função $\psi^{*}$ é crescente, isto é, se $x \leq y$ então $\psi^{*}(x) \leq \psi^{*}(y)$. (Aqui, $x \leq y$ denota o ordem parcial componente a componente em $\mathbb{R}_{+}^{n}$ )

Demonstração Ver Norros [16]. 


\section{A.3 Filtragens associadas aos Processos Pontuais}

Seja $(\Omega, \mathfrak{F}, P)$ um espaço de probabilidade, e sejam $\left(T_{n}, n \geq 1\right)$ e $\left(Z_{n}, n \geq 1\right)$ duas sequência de variáveis aleatórias definidas em $(\Omega, \mathfrak{F})$. Os $T_{n}$ tomam valores reais e os $Z_{n}$ tomam valores em um espaço $(E, \mathcal{E})$. Além disso suponhamos que $T_{n}<\infty$ e $0<T_{n} \leq T_{n+1}$. A sequência $\left(T_{n}, Z_{n}\right)$ é chamada processo pontual com valores em $E$.

Associado ao processo pontual $\left(T_{n}, Z_{n}\right)$ temos o processo de contagem

$$
N_{t}(A)=\sum_{n \geq 1} 1\left(T_{n} \leq t\right) 1\left(Z_{n} \in A\right), A \in \mathcal{E}
$$

e a filtragem

$$
\mathfrak{F}_{t}=\sigma\left(N_{s}(A) ; 0 \leq s \leq t, A \in \mathcal{E}\right) .
$$

Teorema A.3.1 Seja $\left(T_{n}, Z_{n}\right)$ um processo pontual com valores em E definido em $(\Omega, \mathfrak{F})$. Então:

a) Para todo $n \geq 1, T_{n}$ é um $\mathfrak{F}_{t}$-tempo de parada;

b) A filtragem $\mathfrak{F}_{t}$ é contínua à direita, i.e $\mathfrak{F}_{t}=\bigcap_{h>0} \mathfrak{F}_{t+h}$;

c) $\mathfrak{F}_{T_{n}}=\sigma\left(T_{i}, Z_{i} ; 0 \leq i \leq n\right)$.

Demonstração. Ver Bremaud [6].

Teorema A.3.2 Seja $S$ um $\mathfrak{F}_{t}$-tempo de parada. Então

$$
\mathfrak{F}_{S} \cap\left\{T_{i} \leq S<T_{i+1}\right\}=\mathfrak{F}_{T_{i}} \cap\left\{T_{i} \leq S<T_{i+1}\right\}
$$

Demonstração. Ver Bremaud [6].

O teorema pode ser lido como segue: "Se conhecemos que $\left\{T_{i} \leq S<T_{i+1}\right\}$, então $\mathfrak{F}_{S}$ contem a mesma informação que $\mathfrak{F}_{T_{i}}{ }^{\prime}$

\section{Observação 7}

Nosso caso de interesse nesta dissertação é quando $S=t$ e os tempos que definem o processo pontual são dadas pelas estatística de ordem. Assim o teorema diz

$$
\mathfrak{F}_{t} \cap\left\{T_{(i)} \leq t<T_{(i+1)}\right\}=\mathfrak{F}_{T_{(i)}} \cap\left\{T_{(i)} \leq t<T_{(i+1)}\right\}
$$




\section{Referências Bibliográficas}

[1] E. Arjas, Survival models and martingale dynamics, Scandinavian Journal of Statistics 16 (1989), no. 3, 177-225. 10, 35

[2] E. Arjas and I. Norros, Life lengths and association: a dynamic approach, Mathematics of Operations Research 9 (1984), 151-158. 38

[3] A. Balkema and Laurens de Haan, Residual life time at great age, Annals of Probability 2 (1974), 792-804. 48

[4] R Barlow and F Proschan, Statistical theory of reliability and life testing: Probability models, fourth ed., Holt, Rinehart and Winston, New York, 1974. 1

[5] R. Barlow and F. Proschan, Importance of systems components and fault tree events, Stochastic Process and Applications 3 (1975), 153-173. 29

[6] P Bremaud, Point processes and queues: Martingale dynamics, Springer-Verlag, New York, 1981. $56,58,61,63,68,70$

[7] V. Bueno, Minimal repair redundancy for a coherent system in its signature representation, American Journal of Operations Research 1 (2011), p. 8/2011.11002-15. 37

[8] V.C. Bueno, Minimal standby redundancy allocation in a $k$-out-of-n: F system of dependent components, European Journal of Operational Research 165 (2005), 786-793. 35

[9] V.C. Bueno and J. Menezes, Pattern's reliability importance under dependence condition and different information levels, European Journal of Operational Research 177 (2007), 354-364. 29

[10] L. De Haan and A. Ferreira, Extreme value theory: An introduction, Springer series in operations research and Financial engineering, New York, 2010. 46

[11] M. Degen, On multivariate generalized pareto distributions and high risk scenarios, Diploma Thesis, ETH Zurich,http://www.math.ethz.ch/ degen/diploma-thesis.pdf (2006). 48

[12] R.A Fisher and L.H.C Tippett, Limiting forms of the frequency distribution of largest or smallest member of a sample, Proc. Cambridge Philos. Soc 24 (1928), no. 1, 180-190. 45

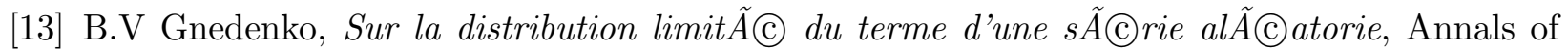
Mathematics 44 (1943), 423-453. 45

[14] Mukerjee H. Kochar, S. and F. Samaniego, The signature of a coherent system and its application to comparisons among systems, Naval Research Logistic 46 (1999), 507-523. iii, v, 5 
[15] Balakrishnan N. Navarro, J. and F.J. Samaniego, Mixture representation of residual lifetimes of used systems, Journal of Applied Probability 45 (2008), 1097-1112. iii, v, 5

[16] I. Norros, A compensator representation of multivariate life length distributions, with applications, Scandinavian Journal of Statistics 13 (1986), no. 2, 99-112. 38, 67, 69

[17] J. Pickands, Statistical inference using extreme order statistics, Annals of Statistics 3 (1975), 119-131. 48

[18] P. Protter, Stochastic integration and differential equations-a new approach, Springer-Verlag, New York, 1990. 58, 59, 62

[19] Balakrishnan N. Navarro J. Samaniego, F., Dynamic signature and their use in comparing the reliability of a new and used systems, Naval Research Logistic 56 (2009), 577-596. 5, 6, 8, 9

[20] F. Samaniego, System signature and their applications in engineering reliability, International Series in Operation Research and Management Science 110. iii, v, 1, 2, 3

[21] F. Samaniego, On the closure of ifr class under formation of coherent systems, IEEE. transactions on reliability R-34 (1985), 69-72. 1

[22] F.J. Samaniego, On comparison of engineered systems of different sizes, in Proceeding of the 12th Annual Army conference on Applied Statistics. Aberdeen Proving Ground, Army Research Laboratory. 5

[23] M. Shaked and A. Suarez-Llorens, On the comparison of reliability experiments based on the convolution order., Journal of American Statistical Association 98 (2003), 693-702. 5 